\title{
Legal regime, Size, and Liquidity factors in Asset Pricing
}

\author{
Bruce Hearn* \\ King's College London \\ Department of Management \\ 150 Stamford St, London SE1 9NH \\ Email: bruce.hearn@kcl.ac.uk \\ Kate Phylaktis \\ Sir John Cass Business School \\ Faculty of Management \\ 106 Bunhill Row, London EC1Y 8TZ \\ Email: k.phylaktis@city.ac.uk \\ Jenifer Piesse \\ King's College London \\ Department of Management \\ 150 Stamford St, London SE1 9NH \\ Email: jenifer.piesse@kcl.ac.uk \\ and University of Stellenbosch, South Africa
}

\begin{abstract}
This study introduces a new legal regime factor into the international valuation literature which exploits the differences between civil and common law origin countries. This builds directly on the concept that institutions both build and shape culture and economic outcomes and takes account of the pervasive differences between civil and common law countries worldwide La Porta et al (1998, 2002). This study contrasts the abilities of three prominent liquidity constructs, namely Amihud (2002) price-impact, Liu (2006) trading speed and volume-based turnover, in explaining the total trading costs in a sample of 62 equity markets spanning developed and developing countries as well as aggregated worldwide civil and common law universes. The evidence reveals that differences in legal origin of markets exert a pervasive effect on the liquidity generating process that transcends institutions across markets. Furthermore a world market universe is created from the constituent stocks of the top tier equity market indices from 60 countries worldwide leading to the construction of size and liquidity returns-based factors and a new legal regime factor. The results indicate that the four-factor legal regime CAPM outperform both other pricing models.
\end{abstract}

JEL classification: G11, G12, G15, O55

Keywords: Legal Origin, Asset pricing, International Financial Markets

Corresponding author: School of Management, Ken-Edwards Building, University of Leicester, UK. LE1 7RH. Tel: 44(0)116 252 5520. Email: bruce.hearn@kcl.ac.uk

The authors wish to thank the participants of the $2^{\text {nd }}$ Emerging Markets Group conference, Cass Business School, London, May 2008, and of the International Finance Conference, Trinity College Dublin for useful comments. 


\section{INTRODUCTION}

The origin of country's legal and regulatory systems has well documented effects on the institutional design and cultural makeup of that nation (La Porta et al $(2002,2008)$ ). While common law tradition traces it's origins back to the English system of establishing legal precedent on a case-by-case basis the civil codes of France, Germany and to a much lesser extent Scandinavia trace their origins to Roman Law where law was established through a codified system or principles and emphasis was placed on courts interpretation of these guidelines (La Porta et al, 2008). This is particularly prevalent in Napoleonic French civil code where the judiciary were relegated to a largely administrative role in the interpretation of codified statutes and through centuries of colonisation, conquest and influence this spread both throughout France's colonial empire as well as to Spain and Portugal and by virtue of their empires on to Latin America and parts of Asia (La Porta et al, 2008). However while there has been much intermixing between the systems with characteristically common law country's such as US enacting essentially civil law codified legislative measures, an example being the recent Sarbanes-Oxley Act, and a considerable uptake of corporate governance regulations in civil code country's that is more common law in nature the institutional differences between the two systems are largely distinct leading to fundamentally very different development outcomes (La Porta et al, 2008). Consequently I ask whether the differences between civil and common law regimes exert a pervasive effect on both the liquidity and returns generating processes across country's adhering to their principles.

The application of standard asset pricing theory dictates that the cross-section of expected stock returns are related to returns sensitivities to state variables that are themselves linked to investors overall welfare (Pastor and Stambaugh, 2003). Assets whose lowest returns accompany unfavourable shifts in that welfare must compensate investors for the loss of value while holding the asset. While Fama and French (1993) originally proposed that variations in size as well as accounting book to market value of stocks across a universe constitute state variables there is considerable evidence in the literature of liquidity being such a variable (Pastor and Stambaugh (2003); Liu (2006)). However the existence of persistent differences between civil as opposed to common law institutions and the ability of these to be propagated on an inter generational basis once transplanted to nations thus forming a pervasive constituent of national culture is also likely to be such a state variable. Differences in legal origin transcend institutional features through affecting the design of corporate bankruptcy laws, shareholder activism, information disclosure and principles of governance in a systematic way across the world between civil and common law legal origin country's. As such I ask whether differences in cross sectional expected returns can be better explained by fluctuations in aggregate market size, liquidity and legal regime effects as opposed to size and liquidity factors alone. 
Liquidity as a concept is very hard to define largely because its representative characteristics transcend a number of transactional properties of markets including tightness, depth, resiliency (Lesmond, 2005) and information (O'Hara, 2003). The literature has traditionally been limited in only employing constructs capturing only one dimension of a multidimensional phenomenon. This typically centres on variants of the bid-ask spread (quoted or effective) in Amihud and Mendelsen (1986), the turnover measure of Datar et al. (1998), or measures relating to the price impact arising from traded volume such as Amihud (2002) and Pastor and Stambaugh (2003). However there is very little published research concerning measures capturing the trading speed dimension of liquidity, defined as the ability to transact large quantities quickly with little price impact (Liu (2006) and Pastor and Stambaugh (2003)). Furthermore there are serious concerns over existing one-dimensional constructs ability to fully capture liquidity risk and over their inaccurate estimation of the dimension they are intended to model Pastor and Stambaugh (2003) and Amihud (2002). Equally deficiencies in the application of the bid-ask spread construct have been highlighted in Lee (1993) where evidence reveals that many large trades occur outside the bid-ask spread while many small trades are undertaken within it leading to potential bias. Further concerns over the application of one-dimensional measures focus on their being undefined in the presence of extremes of illiquidity as is a frequent occurrence in smaller regional markets (Lesmond, 2005). A more recent measure developed in Liu (2006) captures the trading speed dimension of liquidity which is defined as the standardized turnover-adjusted number of zero trading volumes over the past twelve months. It is multi-dimensional in nature, capturing effects relating to trading speed, trading quantity and trading cost, with an emphasis on trading speed, outlined as the continuity of trading and the potential delay in executing an order (Liu, 2006).

The literature concerning the inclusion of liquidity as a priced state variable within a valuation framework is very recent. Pastor and Stambaugh (2003) find strong evidence from US stock data that market-wide liquidity is a priced state variable and that the liquidity premium should be positive. The study applied the innovations of a price impact measure of liquidity to sort stocks within a universe into decile portfolios with the market aggregate premium being formed in the difference between returns of the highest and lowest liquidity deciles. The explanatory power arising from inclusion of the liquidity factor were studied through the contrast of a four factor capital asset pricing model (CAPM) including market, size, price-to-book value and the new liquidity factor against the Fama and French (1993) three factor model and the CAPM. Stocks with higher sensitivity to aggregate liquidity stocks compensate investors with higher expected returns. Evidence is also found that small stocks have greater sensitivities to liquidity innovations than large stocks. Pastor and Stambaugh (2003) note that intuitively it could be expected that small and illiquid stocks are those most 
affected by market aggregate drops in liquidity thereby precipitating investors to "flee" to assets with higher liquidity. However their findings also show that size and liquidity are not the sole determinants of liquidity betas. This finding is reinforced by the argument explaining why stocks with a high liquidity beta are not necessarily illiquid. Investor preferences when there are market aggregate falls in liquidity are also likely to focus on rival bonds markets. In order to increase portfolio holdings in bonds investors may seek to sell liquid stocks in order to save on transactions costs. Consequently in this scenario the price reaction to aggregate liquidity changes is stronger for more liquid stocks. Equally prices of liquid stocks could have greater sensitivity to aggregate liquidity shocks if such stocks are held in greater proportions within the portfolios of liquidity-conscious investors. As such Pastor and Stambaugh (2003) find little basis for liquidity betas to bear a simple relation to stock size and liquidity. Liu (2006) builds on this background in first using a new liquidity construct to estimate stock liquidity and then including this factor within a two factor augmented capital asset pricing model (CAPM). While the additional liquidity factor offers strong performance in explaining the cross section of US stock returns the findings are in contradiction to the earlier findings of Pastor and Stambaugh as the liquidity premium solely subsumes the documented anomalies such as size and the book-to-market effects from Fama and French (1993).

The literature regarding liquidity and its effects on stock returns is itself very recent with very little focus on the comparison between the relative robustness of measures in capturing effects. Lesmond (2005) provides a comparative study of the relative robustness of five well established metrics across 23 emerging market countries and Hearn and Piesse (2010) undertake a similar exercise for the smaller West African region. However while no worldwide study has been undertaken there has been no focus on the differences in liquidity within the context of differences between civil and common law markets. As such a shortfall in the literature is in the direct contrasting of these measures in explaining the total trading costs, defined as the bid-ask spread plus the trading commission levied by exchange for both buy and sell sides of a trade. Consequently I ask which of these measures best explains total trading costs and use the most robust construct in forming liquidity valuation factor to further assess the relationship between liquidity and stock returns.

In this paper I present evidence that the ubiquitous turnover ratio is the most consistently robust measure across civil code markets while that of Liu is preferable for common law markets. I also find substantial evidence that size and liquidity returns-based factors are fundamentally important in valuation while the new proposed legal regime factor is important to a lesser degree. Furthermore the legal regime valuation factor is especially prevalent in markets with well documented very strong or very weak investor protection 
legislation. It also adds credibility to the continued use of the mean-variance paradigm in valuation.

The paper is structured as follows. Section 2 reviews the institutional features of common and civil law equity markets and documents the construction of liquidity measures. Section 3 contrasts the different liquidity metrics through regression and maximum likelihood analysis and then details the construction of the four valuation returns-based factors: market, size, liquidity and legal regime. Section 4 outlines the CAPM methodology and the four factor augmented CAPM valuation model while section 5 details the results, first from the differentiation tests between the various measures and then for the application of the valuation model. The final section concludes.

\section{MARKET INSTITUTIONS AND LIQUIDITY MEASUREMENT}

This section documents the construction of measures used to capture liquidity effects of stocks. All measures were applied to all stocks across the market universe, itself made up from the constituents of the top tier blue chip indices in each market which conforms to the concept of these stocks being most likely to feature on menus of international portfolio managers for diversification. These are also the most likely candidate stocks to conform with the assumption of international asset market integration which is critical to the application of CAPM valuation model.

\subsection{Liquidity constructs}

\section{The Bid Ask spread and commission cost}

The Bid Ask spread and commission cost: The data on the end of month bid and ask quotes were collected from Datastream. The bid-ask spread is calculated using the average of the available monthly quotes and incorporates at a minimum a single month's quote for that month. The average bid-ask spread spanning the quarter is used for the estimate of the spread. This procedure minimizes outlier problems and averages out the recording of either highs or lows in quotes resulting from monthly sampling. Following Lesmond (2005) bid-ask spreads that exceed $80 \%$ are trimmed as these are potentially errors. The monthly quoted spread is defined as:

Quoted spread $_{M}=1 / 2\left[\left(\frac{\mathbf{A s k}_{M}-\mathrm{Bid}_{M}-}{\mathbf{A} s k_{M}+B i d_{M} \searrow 2}\right)+\left(\frac{\boldsymbol{A} s k_{M-1}-B i d_{M-1}-}{\mathbf{A} s k_{M-1}+B i d_{M-1} \searrow 2}\right)\right]$

In order to estimate the total trading transaction costs, the costs associated with a round trade (both buy and sell legs) are added to the quoted spread for each month. Brokerage and Exchange fees are calculated from the fee schedules detailed in Appendix Table 1. When a percentage commission fee is not provided the maximum fixed cost is applied to the aggregate daily traded value data. 


\section{Turnover}

Daily trading volume data and shares outstanding data was obtained from Datastream. At first glance it is apparent that there is considerable variation in this measure on an intramarket basis. This reflects the substantial differences in both liquidity and turnover for many of the companies within each market. Any turnover statistics that exceed $100 \%$ of the shares outstanding in any month are trimmed from the sample. The shares-outstanding is determined at the start of the year and remains constant for the 12 months thereafter. The daily turnover measure is defined as:

$1 / D_{M} \sum_{t=1}^{M}$ rolume $_{t} /$ shares - outstanding_-

where $D_{M}$ is the number of days in the month, M.

\section{Amihud (2002) measure}

Daily price and volume data are sourced from Datastream. The daily security prices are scanned for data errors, omissions and delistings. Following the procedure outlined in Lesmond (2005) the prices are used calculate daily returns. To control for return outliers, a data error filter eliminates daily prices that are $+/-50 \%$ of the prior day's price and that day's price as well as previous day's price are deleted from sample. Equally if zero volume occurs on day $t$, then that day is deleted from average. Finally the measure is multiplied by $10^{6}$ as undertaken in Amihud (2002) in order to provide a common representation of measures and facilitate comparison. The Amihud measure is defined as:

$1 / D_{M} \sum_{t=1}^{M} R_{t} \mid /$ Price $_{t} \times$ Volume $_{t}^{-}$

\section{Liu (2006) measure}

Daily price and volume data are collected from Datastream. The measure is derived from the recent work of Liu (2006) and is defined as $\mathrm{LM}_{\mathrm{x}}$ which is the standardized turnover-adjusted number of zero daily trading volumes over the prior $\mathrm{x}$ months $(\mathrm{x}=1,6,12)$ i.e.

$L M_{x}=\left[\right.$ Number of zero daily volumes in prior x months $\left.\_\frac{1 / \mathrm{x} \text { month turnover }}{\text { Deflator }}\right] * \frac{21 x}{\text { NoTD }}$

where $\mathrm{x}$ month turnover is the turnover over the prior $\mathrm{x}$ months, calculated as the sum of the daily turnover over the prior $\mathrm{x}$ months, daily turnover is the ratio of the number of shares traded on a day to the number of shares outstanding at the end of the day, NoTD is the total number of trading days in the market over the prior x months, and Deflator is chosen such that, 
$0<\frac{1 / \text { month turnover }_{-}^{-}}{\text {Deflator }}<1$

for all sample stocks ${ }^{1}$. Given the turnover adjustment (the second term in brackets in first expression), two stocks with the same integer number of zero daily trading volumes can be distinguished: the one with the larger turnover is more liquid. As such the turnover adjustment acts as a tie-breaker when sorting stocks based on the number of zero daily trading volumes over the prior $\mathrm{x}$ months. Because the number of trading days can vary from 15 to 23 , multiplication by the factor (21x/ NoTD) standardizes the number of trading days in a month to 21 which makes the liquidity measure comparable over time. LM1 can be interpreted as the turnover-adjusted number of zero daily trading volumes over the prior 21 trading days, which is the approximate average number of trading days in a month. The liquidity measure, $\mathrm{LM}_{\mathrm{x}}$ is calculated at the end of each month for each individual stock based on daily data. Daily data is available for all markets across entire sample period.

\subsection{Data: Sources}

Daily stock closing, bid and ask prices, total number of shares outstanding, traded volumes, dividend per share in local currency and converted into UK£ were obtained for all markets from Datastream. These data were supplemented by bid and ask price data sourced from Bloomberg for the markets of Bulgaria, Jamaica and Russia where data was unavailable from Datastream. These data formed the basis of calculation of the daily return variance, or volatility, market capitalization, defined as total number of shares outstanding multiplied by daily closing price, and various liquidity constructs. The total returns series for each stock were sourced direct from Datastream for all markets. Exchange rate and UK- Gilt/Treasury yield data are sourced from Datastream. The one-month UK-Gilt/Treasury Bill yield rate represents the risk free rate although this is adjusted to take account of monthly excess returns as opposed to the quoted equivalent annualised rates. The conversion of the total returns series and prices into sterling and the use of UK - Gilt/Treasury yield rate assumes long term parity between the local currency and sterling. This is reasonable given many of the developing countries within the sample have experienced periods of severe macroeconomic instability and hyperinflation during the sample period. The conversion to UK£ sterling also facilitates international comparison given the sizeable role of the London financial market in emerging market investment (Froot et al, 2001).

\subsection{Data: Summary statistics relating to liquidity measures}

\footnotetext{
${ }^{1}$ In line with Liu (2006) a deflator of 1,000 is used in constructing estimates for LM1
} 
The descriptive statistics across the constituent stocks of the top tier blue chip indices of each of the world markets considered in this paper are given in Table 1. The most striking difference is between developed and emerging markets with the former having much less price-rigidity (lower percentage daily zero returns), lower bid-ask spreads and larger traded volumes. This is exemplified by the markets within the Developed Europe category having percentage daily zero returns values generally between 10 and $20 \%$, with prominent exceptions of Ireland and Iceland, while those within the Emerging Europe category are as high as $80.44 \%$ (Russia) and $77.51 \%$ (Slovakia). Similarly the emerging market regions of Africa, where the very top tier elite indices have been used given the well documented extremes of illiquidity in this region (Hearn and Piesse (2009); Hearn (2009) and Hearn and Piesse (2010)), and Latin America have very high bid-ask spreads and daily percentage zero returns. The very recently established markets within the Middle Eastern region also have very high illiquidity although prominent exceptions are Israel and Saudi Arabia where liquidity is considerably higher than the surrounding region. Overall the evidence would infer that civil code markets are smaller and more illiquid than their common law counterparts.

\section{Table 1}

\section{LIQUIDITY MEASURE CONTRASTS AND FACTOR GENERATION}

This section contrasts the three Liquidity metrics in their relative ability to explain the total trading costs (bid-ask spread plus commissions) dependent variable through regressions. This is further extended by an application of maximum likelihood analysis techniques in their comparative ability to explain the total trading costs data generating process. Following this justification of the measure that is to be used to sort stocks into portfolios based on their relative illiquidity the returns-based valuation factors are constructed.

\subsection{Spearman's rank correlation}

Given the variables have different measurement scales a non-probability distribution limited correlation, the Spearman's rank, is used for the measurement of association. In addition to the bid-ask spread and the three liquidity measurement constructs, turnover, Liu and Amihud variables, price, daily price return volatility, traded volume, and market capitalization measures are introduced as control variables in line with those used in the investigations for price of trading immediacy undertaken by Stoll (2000). The rationale for the inclusion of these variables is based on order processing and inventory considerations of traders, albeit in a study undertaken by Stoll (2000) in the US equity market. Increases in volume and firm size increase the probability of locating a counterparty, thereby mitigating the risk of accepting and holding inventory risk. The stock price volatility, in local currency terms, provides an indication of the risk of adverse price changes of a stock placed on a trader's 
inventory, while the price measure itself controls for the effect of discreteness and is an additional proxy for risk in that low price stocks tend to be riskier.

The Spearman's Rank correlations were estimated between variables across individual universes for each separate market in turn (results reported in Appendix 2) and in aggregate for the World civil code and World common law universes (results in Table 2). The evidence from Table 2 reveals that there are subtle differences in the inter-relationships between the variables between the civil and common law universes. While the size, sign and direction of relationships between variables tends to be similar those in the common law universe are noticeably larger in absolute size. An additional significant difference is the size and significance of the large negative relationship between bid-ask spread and price as well as the Liu measure which while being in line with theory is discernable in the common law but not civil code universe. Similarly the minimal relationship between firm size and bid-ask spread in civil code as opposed to common law universe is counter-intuitive. Equally the markedly lower level of relationship between bid-ask spread and all the other variables in the civil code as opposed to common law universe is likely indicative of very different structure of the two types of markets and different data generating processes for aggregate liquidity.

\section{Table 2}

\subsection{An assessment of liquidity measures ability in explaining total trading costs}

A direct measure of association between the total trading costs and the market control variables, which Stoll (2000) defines as price, traded volume, volatility, and firm size or market capitalization, as well as the various liquidity measures are provided in this section. Following Stoll (2000) price is defined as a proxy for risk, as lower priced stocks tend to be riskier, and controls for the effects of price discreteness. Volume and firm size proxy for order processing and inventory considerations. Increases in volume and firm size increase the probability of locating a trade counter party, which reduces risk. Volatility measures the risk of adverse price changes because of stock put into inventory (see Lesmond, 2005). Three sets of multivariate fixed effects regressions were estimated for each of the individual markets in turn as well as for the aggregated World Civil, Common and overall universe with the dependent variable in each cases being the stock's total trading costs. The first set concerns the relationship between total trading costs and the Stoll market control variables only. The second set concerns total costs and the Stoll control group as well as each of the three individual liquidity measurement variables (turnover, Amihud and Liu) in turn so as to differentiate between the liquidity variables through any incremental benefits with the inclusion of one as opposed to another. The third set of regressions (or grand regression) concerns the relationship between total costs and the combination of the Stoll control variables in addition to all three liquidity measures. Differentiation can be made through the 
level of explanatory power, or $\mathrm{R}^{2}$ terms, and to a lesser extent the statistical significance of variables assessed by confidence levels of t-statistics. In every case price, volume and firm size are natural-log scaled in line with Stoll (2000).

\subsection{Vuong likelihood ratio test}

The maximum likelihood method of Vuong (1989) (see Appendix 3 for description of empirical methods employed) offers a robust alternative to regression-based methods that merely test the level of association between the Stoll market control variables and the various liquidity constructs against the total trading costs with relative strength being assessed on the basis of statistical significance and explanatory power. The tests proposed in Vuong (1989) use a one-sided Z-probability distribution to assess the confidence levels arising from the study of which liquidity measures better explain the data generating process behind the total trading costs.

\subsection{Valuation Factor construction}

Four returns-based valuation factors are constructed which form the inputs to the CAPM modelling methodology. The first, the market premium or factor, is simply the average excess returns across all stocks within the world sample group universe. I have used the simple equally weighted mean as opposed to a market capitalization weighted average as this would induce a source of potential bias through the market factor being dominated towards the larger markets such as US, Singapore, Japan and Australia as well as Europe thus failing to truly represent the effects of the smaller emerging markets such as Chile, Jamaica, Argentina, Morocco and South Africa.

The size and liquidity factors are formed through the sequential sorting of stocks first into three capitalization portfolios, namely "small", "medium" and "big", which are then sorted in turn into three illiquidity portfolios, namely "high", "medium", "low". Portfolio rebalancing is undertaken each December for the duration of the sample period, i.e. January 2000 to June 2009. The size ("SMB") valuation factor is formed from the equally weighted mean excess returns from the three small size portfolios minus the mean of the three big size portfolios. The liquidity ("ILLIQ") valuation factor is formed from the equally weighted mean of the three high illiquidity portfolios, one being in each of the original size sorted portfolios, minus the mean of the three low illiquidity portfolios. Stocks are ranked in accordance to their annual mean of liquidity measure, which has been chosen on the basis of the results from the regression-based and maximum likelihood tests. Stocks ranked and sorted into portfolios each December are held for a further twelve months until portfolio rebalancing the following year. 
The third valuation factor, attributed to differences between the legal origins of the various individual markets regulatory regimes, i.e. "LEGAL REGIME", is simply the equally weighted mean of excess returns across common law countries minus the mean of returns from across civil law markets. As such it captures the simple differences in effects caused by the very different nature of institutions within civil and common law markets. It is calculated on a monthly basis across the entire sample group market universe.

\section{EMPIRICAL VALUATION MODELS}

The size and liquidity augmented three factor CAPM model of Pastor and Stambaugh (2003) follows in the spirit of Fama and French (1993) in augmenting the traditional CAPM with extra size and liquidity returns-based factors which mimic the effects of the hypothesized underlying state variables. I extend this by adding the extra returns-based factor to the sizeilliquidity CAPM and form a four factor (size - illiquidity - legal regime) augmented CAPM. Therefore, the expected excess returns on a portfolio $\mathrm{p}$ of emerging market stocks can be written as

$$
E \boldsymbol{C}_{p t} \supset r_{f t}=\beta_{p} \mathbf{E} \boldsymbol{C}_{n t} \mathcal{-} r_{f t}+s_{i} E \mathbf{Q} M B \ni h_{i}\left(L L I Q \ni z_{i}(L E G A L)\right.
$$

In line with the above this can be transformed in order to test historical data into the following equation:

$$
r_{i t}-r_{f t}=\alpha_{i}+\beta_{i}\left(r_{m t}-r_{f t}\right)+s_{i} S M B_{t}+h_{i} I L L I Q_{t}+z_{i} L E G A L+\varepsilon_{i t}
$$

where the variables are described above and $\varepsilon_{i t}$ is an independently identically distributed (iid) disturbance term. The model is estimated on a time series basis using standard Ordinary Least Squares (OLS) techniques, as opposed to the Fama and Macbeth (1973) rolling cross section approach, with the expectation that the Jensen alpha, or regression intercept, should not be statistically different from zero given the theoretical relationship between an individual portfolios expected returns and those of the market (Markowitz (1959); Sharpe (1964) and Lintner (1965)). However Scholes and Williams (1977) provide evidence against the employment of standard OLS techniques with findings that beta estimations are biased downwards for securities infrequently trading and upwards for those traded more often. Dimson (1979) builds on this evidence in the inefficiency of beta estimation in thinly traded stocks and proposes a correction technique based on the aggregation of betas from lagged and leading regression coefficients. Dimson and Marsh (1983) propose a second correction technique which uses a trade-to-trade method measuring and matching returns between individual stocks or portfolios and the market index between the times of the last trades in successive months. I justify the use of standard OLS techniques here in order to closely follow the literature of Pastor and Stambaugh (2003), Liu (2006) and Martinez (2005) who use these techniques extensively in their studies involving multifactor CAPM models 
capturing liquidity effects. The limitations of standard OLS techniques should be taken into account particularly when they are applied to a very diverse universe of stocks, such as this, as is necessarily used in cross-country comparative studies.

\section{RESULTS}

Essential to the understanding that the differences between common and civil law institutions are both pervasive across and between markets I apply the regression based and maximum likelihood techniques both on an individual market-by-market basis as well as to aggregate World common, civil and overall universes.

\subsection{An assessment of liquidity measures ability in explaining total costs}

The evidence from Table 3 reveals that the explanatory power of the Stoll market control variables and liquidity measurement metrics across individual markets is generally high, although this is subject to considerable variation from adjusted $\mathrm{R}^{2}$ ranging from just over $15 \%$ for Taiwan to over $89 \%$ in Qatar. However there are some significant outliers, where explanatory power is negligible, such as China's Shenzen and Shanghai exchanges and Colombia, where only a very limited period of bid and ask data was available. There is also considerable variation between individual markets in the amount of statistical significance of the various liquidity measures. However these issues notwithstanding the evidence from the three World regressions, namely common law, civil law and overall in the final section of the Table reveals that while the addition of the Liu metric to the regression containing the Stoll control variables causes the largest increase in explanatory power for the World common law market the addition of the turnover measure on it's own is sufficient for the World civil law market. The results for the aggregated World overall universe are similar to those of the World civil law universe where the turnover measure on its own is both significant and causes greatest increase in explanatory power.

\section{Table 3}

\subsection{Vuong maximum likelihood ratio test}

The evidence from the Vuong maximum likelihood ratio analysis in Table 4 provide statistics that are one-sided and directional in their nature. As such a positive statistic that is larger in absolute terms to the test statistic (at a given confidence level) of the one-sided Z-probability distribution indicates that the liquidity measure in the first row of the table is preferable to the variable or measure (shown in second row) for each individual market. Generally the results in this table favour the choice of the Liu estimator as opposed to the price-impact measure of Amihud. Both outperform the ubiquitous turnover measure. This evidence is strengthened from the final World section, where Liu outperforms the Amihud measure, where the latter 
actually performs poorly in contrast to the turnover measure. The turnover metric is especially strong over and above the Amihud and Liu measures for World civil law universe while it is less strong in the case of the World common law universe. At this stage the Liu estimator is preferable in contrast to either turnover or Amihud measures although the evidence is not so clear cut.

\section{Table 4}

\subsection{Summary statistics relating to size-liquidity sorted portfolios and factors}

There is generally little distinguishable difference in either mean returns or standard deviations between size-illiquidity sorted portfolios in Table 5. However there is a slight increase in mean returns of the three small size in contrast to the three large size portfolios indicating the possible presence of a reverse size effect where returns increase as firm size decreases although this is not pronounced. The levels of skewness and kurtosis are minimal across portfolios indicating portfolio returns have little deviation from Normality in their probability distributions lending support to the later application of regression techniques in their modelling. Overall there is little difference in numbers of stocks between the nine sizeilliquidity sorted portfolios with each portfolio having approximately between 470 and 485 stocks each. The differences are most prevalent on the study of the regional makeup of the stocks falling within the portfolios. While there is little difference between world regions in terms of size sorted portfolios it is noticeable that developed markets dominate the low illiquidity portfolios while their emerging counterparts fall largely within the high illiquidity portfolios in each of the three size categories.

\section{Table 5}

The evidence from Table 6 reveals that while mean returns of the market portfolio are positive they are negative for the size (SMB), Illiquidity (HML) and Legal Regime (LEGAL) valuation factors. This provides stronger support for the presence of a reverse size effect where returns decrease as firm size increases. However the negative value of the illiquidity factor is in accordance to theory where returns decrease as illiquidity increases. Equally the very high skewness statistic for the illiquidity factor is rationalised as small highly illiquid stocks are also commonly skewed and leptokurtic (fat tailed probability distributions). The negative sign of the LEGAL factor is harder to interpret. Given the nature of the factor's design, where the returns attributable to civil law markets are deducted from common law markets, the sign would be expected to be positive if indicating a general trend in legal and regulatory regimes towards the markets-orientated common law regulatory system. However the negative sign would indicate that there is a general trend towards the civil law regulatory regimes. Levels of skewness and kurtosis indicate that returns attributable to this factor fit 
within a Normal probability distribution while levels of standard deviation are extremely high in contrast to all other factors.

Correlations between factors are very low, as seen in second section of Table 6, indicating that the factors properly proxy underlying state variables present within the sample market universe. This is further confirmed with the study of the significance in the difference of the time series of mean returns for each factor, denoted by a high and statistically significant t-statistic in the lower portion of Table 6. This provides strong evidence that factors genuinely proxy or mimic underlying state variables and are properly constructed zero cost portfolios. The returns-based factors are shown in Figures 1 to 4.

\section{Table 6}

\section{Figures 1 - 4}

The evidence from the individual market, or country, portfolios in Table 7 reveals that as would be expected standard deviations are generally higher for emerging market regions as compared to developed markets. However the most striking feature is that the most highly illiquid markets have by far the highest values of skewness and to a lesser extent kurtosis. Russia is one such example with skewness of 23.492 and Venezuela is another example in Latin America with value of 33.328. This reflects the evidence from the descriptive statistics earlier in Table 1 where these two individual markets had excessively high percentage zero daily returns and minimal trading volumes.

\section{Table 7}

\subsection{Performance of traditional CAPM against three-factor CAPM}

There is mixed evidence from Table 8 regarding the benefits arising from the inclusion of the additional legal regime factor in CAPM models that already are augmented with size and liquidity factors. The results for the traditional CAPM model indicate that the Jensen alpha term is not significant in the majority of cases. Explanatory power of the one-factor model is also generally high at over $85 \%$. The addition of the size and liquidity factors does increase explanatory power, i.e. the adjusted $\mathrm{R}^{2}$, with the largest of the minimal increases arising from the small size portfolios and the high illiquidity portfolios where the size and liquidity factors have increased statistical significance in relation to the increases in explanatory power. The results from panel $\mathrm{C}$ indicate that the LEGAL factor is only statistically significant for small size - high illiquidity and medium size - high illiquidity portfolios with little general increase in explanatory power over and above that obtained in the three factor size - illiquidity augmented model from panel B. However despite the less than expected performance associated from the inclusion of the legal regime factor the importance of capturing the 
effects of comparative civil versus common law institutions on returns merits its inclusion in the next step of modelling individual market, or country, returns.

\section{Table 8}

\subsection{Modelling market portfolios}

Portfolios representing individual markets were formed on an equally weighted basis and modelled using the traditional CAPM, size-liquidity three-factor CAPM and finally the sizeliquidity-legal regime four factor CAPM with results in Table 9. In contrast to the previous section, where simple size-illiquidity sorted portfolios were used, the evidence supports the inclusion of the LEGAL factor in valuation. Across all 60 individual market portfolios the Jensen alpha terms are generally not statistically significant following the addition of the LEGAL factor and explanatory power is increased, albeit marginally. Prominent exceptions where only the three-factor size and liquidity model is only sufficient are Denmark, Germany and Iceland where explanatory power between three factor model and its four factor counterpart, fractionally decreases. However the sign (or direction) of the LEGAL factor is generally as expected across the individual market portfolios where it is statistically significant. The negative and significant value for the Italian portfolio, in panel 9, is as expected given the civil code nature of this country's legal origins and the poor levels of external shareholder protection leading to dominant block-holders (see La Porta et al (2008) for detail concerning this country). Equally the strong civil code regimes (with substantially weaker shareholder protection and dominant block-holders) of Russia, Bulgaria, Cyprus, Portugal, Egypt, Peru, Venezuela, Philippines and in both Chinese exchanges (Shenzen and Shanghai) have large negative values of LEGAL beta. This is reflective of particularly poor investor protection and regimes where civil code origins are strongly evident. Contrastingly the evidence for individual markets with very strong investor protection and strong common law origins are shown from large positive LEGAL betas. These include US, Canada, New Zealand, Malaysia, Hong Kong, and Thailand with the UK only being significant at a lower level. The contrasting results (in terms of direction and sign) between Hong Kong and the two Chinese markets of Shenzen and Shanghai is interesting and reflects the anomaly that exists in China, where following the demise of British rule in the Hong Kong protectorate in 1997, a "one country two economic systems" regime was installed and the territory's markets continued largely unhindered from central government interference which dominates in Shenzen and Shanghai (da Veiga et al (2008); Tan et al (2008)).

Table 9

\subsection{Cost of equity estimation}


The evidence regarding estimates of costs of equity (see Jagannathan et al (2002) and Harris et al (2003) for full review of estimation techniques) in Table 10 are largely as expected with developed markets generally having the lowest discount rates and emerging markets having the highest. However the extremely low explanatory power of the model when applied to the very small, highly illiquid individual market portfolios of Pakistan, Bangladesh and Jamaica questions the effectiveness of this methodology in such small and illiquid markets where there is considerable segmentation with world markets and world universe. As such these markets were omitted from costs of equity estimation. Russia has the highest cost of equity in Emerging Europe (68.15\%) while Turkey has the highest in the Middle East region (50.49\%).

\section{Table 10}

\section{CONCLUSIONS}

In this paper I contrast the performance of three liquidity measures, namely the turnover ratio, the Amihud price-impact measure, and the trading speed measure of Liu in explaining total trading costs (bid-ask spread plus trading commissions) across a sample of sixty two countries worldwide. The difference between civil and common law countries is particularly evidence with the Liu estimator prevailing in common law and turnover ratio sufficing in civil law markets.

While the differences in liquidity are largely reflective of institutional development between common and civil law countries the more pervasive differences on returns generating processes between each bracket of countries is assessed through the construction of a unique Legal Regime variable accounting for the differences between markets on the basis of their legal origins. This is found to be statistically significant and increasing explanatory power for markets with either very strong common law qualities, such as stringent investor protection laws and rigorously enforced financial reporting and corporate governance standards, such as US, Canada and Hong Kong or very strong civil law characteristics, namely weakened financial reporting and the dominance of block-shareholders, such as China, Bulgaria, Russia and Italy. As such I find evidence that differences between civil and common law regulatory and legal regimes and pervasive across and within markets and their contrasting institutions and institutional development is reflective of these differences in the returns generating process of stocks. Returns are likely to be greater in common law as opposed to civil law markets where there is less uncertainty and greater share-holder activism inferring potentially higher levels of turnover and activity. 


\section{REFERENCES}

Amihud, Y., 2002, "Illiquidity and stock returns: cross section and time series effects," Journal of Financial Markets 5, 31-56.

Amihud, Y. and Mendelson, H. (1986). Asset pricing and the bid-ask spread. Journal of Financial Economics, 17, 223-249.

da-Veiga, B., Chan, F., and McAleer, M. (2008) Evaluating the impact of market reforms on Value-at-Risk forecasts of Chinese A and B shares, Pacific-Basin Finance Journal, 16, 453-475

Datar, V. T., Naik, N. Y. and Radcliffe, R. (1998). Liquidity and stock returns: An alternative test. Journal of Financial Markets, 1, 203-219.

Dimson, E. (1979). Risk measurement when shares are subject to infrequent trading. Journal of Financial Economics, 7, 197-226.

Dimson, E. and Marsh, P. (1983). The stability of UK risk measures and the problem of thin trading. Journal of Finance, 38, 753-783.

Fama, E., and K. French, 1993, "Common risk factors in the returns on stocks and bonds," Journal of Financial Economics 33, 3-56

Fama, E. F. and Macbeth, J. D. (1973). Risk, return and equilibrium: empirical tests. Journal of Political Economy, 81, 607-636.

Froot, K., P. O'Connell, and M. Seasholes, 2001, "The portfolio flows of international investors," Journal of Financial Economics 59, 151-194

Harris, R.S., F.C. Marston, D.R. Mishra, and T.J. O’Brien, 2003, “Ex Ante Cost of Equity Estimates of S\&P 500 Firms: The Choice Between Global and Domestic CAPM," Financial Management, 51-66

Hearn, B., and Piesse, J. (2010). Performance of Size and Liquidity portfolios in West Africa. Applied Financial Economics, forthcoming

Hearn, B. and Piesse, J. (2009) Sector level cost of equity in African financial markets. Emerging Market Review, 10, 257-278

Hearn, B. (2009). Liquidity and valuation in East African Securities Markets. South African Journal of Economics, 77(4), 1-24

Jagannathan, R., and I. Meler, 2002, "Do We Need CAPM for Capital Budgeting?," Financial Management, 55-77

La Porta, R., F. Lopez-de-Silanes, A. Shleifer, and R. Vishny, 2002, "Investor protection and corporate valuation," Journal of Finance 57, 1147-1170

La Porta, R., F. Lopez-de-Silanes, and A. Shleifer, 2008, "The Economic Consequences of Legal Origins," Journal of Economic Literature 46, 285-332

Lee, C. (1993). Market fragmentation and price-execution in NYSE-listed securities. Journal of Finance, 48, 1009-1038. 
Lesmond, D.A., 2005, "Liquidity of emerging markets," Journal of Financial Economics 77, $411-452$

Lintner, J. (1965). The valuation of risk assets and the selection of risky investments in stock portfolios and capital budgets. Review of Economics and Statistics, 17, 13-37.

Liu, W., 2006, “A Liquidity-augmented capital asset pricing model," Journal of Financial Economics 82, 631-671

Markowitz, H. (1959). Portfolio Selection: Efficient Diversification of Investments. New York: John Wiley and Sons; London: Chapman and Hall.

Martinez, M.A., B. Nieto, G. Rubio, and M. Tapia, 2005, “Asset pricing and systematic liquidity risk: An empirical investigation of the Spanish stock market," International Review of Economics and Finance, 14, 81-103

O'Hara, M. (2003) Presidential address: liquidity and price discovery. Journal of Finance, 58, $1335-1354$

Pastor, L. and Stambaugh, R. (2003) Liquidity risk and expected stock returns. Journal of Political Economy, 111, 642-685.

Stoll, H.R., 2000, “Friction,” The Journal of Finance 55, 1480-1514

Sharpe, W. F. (1964). Capital asset prices: A theory of market equilibrium under conditions of risk. Journal of Finance, 19, 425-442.

Scholes, M. and Williams, J. T. (1977). Estimating betas from nonsynchronous data. Journal of Financial Economics, 5: 309-327.

Tan, L., Chiang, T. C., Mason, J. R., and Nelling, E. (2008) Herding behaviour in Chinese stock markets: An examination of A and B shares. Pacific-Basin Finance Journal, 16, 61-77

Vuong, Q., 1989, "Likelihood ratio tests for model selection and non-nested hypotheses," Econometrica, 307-333 
Table 1 Summary Statistics

This table presents summary statistics for the sample group markets. Datastream provides the daily prices, volume and market capitalization information. Start refers to the beginning date of the daily security return data up to the final year 2009. Price is the average of daily prices over each month and is stated in domestic currency and converted to $£$ UK using the average exchange rate for each month and country. Volume is the average of the daily trading volume over each month and is stated in thousands. Market capitalization is measured as of 1 January for each country and is equity market value for each firm expressed in millions of local currency or £UK. The £UK market capitalization is derived using the end of month exchange rate for each country and month. Square parentheses indicate median values for each variable.

\begin{tabular}{|c|c|c|c|c|c|c|c|c|c|}
\hline \multirow[b]{2}{*}{ Country } & \multirow[b]{2}{*}{ Start } & \multirow[b]{2}{*}{$\begin{array}{l}\text { Zero Return } \\
(\%)\end{array}$} & \multicolumn{4}{|c|}{ Local market } & \multicolumn{3}{|c|}{ £UK equivalent } \\
\hline & & & Price & $\begin{array}{l}\text { Volume } \\
\text { (thousands) }\end{array}$ & $\begin{array}{l}\text { Std. Dev. } \\
(\%)\end{array}$ & Mkt. Cap. (m) & Price & Mkt. Cap. (m) & $\begin{array}{l}\text { Bid-Ask spread } \\
(\%)\end{array}$ \\
\hline \multicolumn{10}{|c|}{ Europe Developed } \\
\hline Austria & 1994M12 & $32.96[19.05]$ & $\begin{array}{l}40.28 \\
{[17.19]}\end{array}$ & $\begin{array}{l}2586.4 \\
{[320.5]}\end{array}$ & $\begin{array}{l}0.0192 \\
{[0.0146]}\end{array}$ & $753.3[161.58]$ & $27.44[12.16]$ & $524.75[102.89]$ & $0.0559[0.0126]$ \\
\hline Belgium & 1994M12 & $11.77[4.55]$ & $\begin{array}{l}65.51 \\
{[42.4]}\end{array}$ & $\begin{array}{l}8833.49 \\
{[1862.3]}\end{array}$ & $\begin{array}{l}0.018 \\
{[0.0148]}\end{array}$ & $5369.3[2029.66]$ & $45.86[27.19]$ & $\begin{array}{l}3721.33 \\
{[1329.66]}\end{array}$ & $0.0047[0.0039]$ \\
\hline Denmark & 1994M12 & $28.90[19.05]$ & $\begin{array}{l}1980.63 \\
{[117.34]}\end{array}$ & $\begin{array}{l}5276.26 \\
{[1145.1]}\end{array}$ & $\begin{array}{l}0.0202 \\
{[0.016]}\end{array}$ & 11343.69 [1233.87] & $184.51[9.96]$ & $1064.51[104.41]$ & $0.0177[0.0109]$ \\
\hline Finland & 1994M12 & $20.09[15.00]$ & $9.73[7.68]$ & $\begin{array}{l}19609.32 \\
{[2577]}\end{array}$ & $\begin{array}{l}0.0214 \\
{[0.0177]}\end{array}$ & $2436.6[283.63]$ & $6.79[5.22]$ & $1659.66[187.76]$ & $0.0162[0.0075]$ \\
\hline France & 1994M12 & $5.91[4.35]$ & $\begin{array}{l}41.86 \\
{[36.04]}\end{array}$ & $\begin{array}{l}47295.92 \\
{[25613.4]}\end{array}$ & $\begin{array}{l}0.0213 \\
{[0.0184]}\end{array}$ & $\begin{array}{l}15341.07 \\
{[10173.23]}\end{array}$ & $28.76[24.77]$ & $\begin{array}{l}10573.83 \\
{[7190.9]}\end{array}$ & $0.0039[0.0025]$ \\
\hline Germany & 1994M12 & $12.90[4.76]$ & $\begin{array}{l}32.28 \\
{[23.74]}\end{array}$ & $\begin{array}{l}6551.87 \\
{[330.3]}\end{array}$ & $\begin{array}{l}0.0211 \\
{[0.0183]}\end{array}$ & 5731.55 [1115.69] & $22.56[15.81]$ & 3934.15 [706.64] & $0.0103[0.0071]$ \\
\hline Iceland & 2000M12 & $49.49[42.86]$ & $\begin{array}{l}70.18 \\
{[26.93]}\end{array}$ & $\begin{array}{l}35231.66 \\
{[4818.4]}\end{array}$ & $\begin{array}{l}0.0195 \\
{[0.0158]}\end{array}$ & 16591.75 [7168.89] & $4.80[0.21]$ & $139.88[92.21]$ & $0.0449[0.0106]$ \\
\hline Ireland & 1994M12 & $45.98[36.36]$ & $5.61[2.42]$ & $\begin{array}{l}11740.65 \\
{[2836.1]}\end{array}$ & $\begin{array}{l}0.0293 \\
{[0.0184]}\end{array}$ & $1435.02[173.79]$ & $3.28[1.48]$ & 829.28 [116.48] & $0.0532[0.0229]$ \\
\hline Italy & 1994M12 & $14.98[8.7]$ & $7.57[3.78]$ & $\begin{array}{l}2614.81 \\
{[784]}\end{array}$ & $\begin{array}{l}0.0218 \\
{[0.018]}\end{array}$ & 187.41 [104.05] & $5.12[2.61]$ & $129.92[74.42]$ & $0.0168[0.0099]$ \\
\hline Luxembourg & 1994M12 & 28.91 [22.73] & $\begin{array}{l}70.16 \\
{[48.41]}\end{array}$ & $639.63[8.4]$ & $\begin{array}{l}0.0195 \\
{[0.0147]}\end{array}$ & $4787.94[401.36]$ & $48.8[35.66]$ & $3366.06[278.01]$ & $0.0131[0.0106]$ \\
\hline Netherlands & 1994M12 & $19.98[13.04]$ & $\begin{array}{l}56.43 \\
{[13.8]}\end{array}$ & $\begin{array}{l}21320.92 \\
{[1124.9]}\end{array}$ & $\begin{array}{l}0.0229 \\
{[0.0174]}\end{array}$ & 7231.63 [206.02] & $25.64[9.38]$ & 3944.65 [133.41] & $0.0200[0.0083]$ \\
\hline Norway & 1994M12 & 20.64 [14.29] & $\begin{array}{l}70.37 \\
{[36.6]}\end{array}$ & $\begin{array}{l}36182.82 \\
{[6513.3]}\end{array}$ & $\begin{array}{l}0.0289 \\
{[0.0223]}\end{array}$ & $13055.52[2788.61]$ & $5.93[2.98]$ & $1115.41[243.8]$ & $0.0198[0.0096]$ \\
\hline Portugal & 1994M12 & $27.55[20]$ & $4.02[2.76]$ & $\begin{array}{l}33687.49 \\
{[2140.9]}\end{array}$ & $\begin{array}{l}0.0207 \\
{[0.0156]}\end{array}$ & $1630.01[119.75]$ & $2.78[1.92]$ & 1123.29 [81.95] & $0.022[0.0103]$ \\
\hline Slovenia & 1996M04 & $10.74[8.7]$ & $\begin{array}{l}76.73 \\
{[23.3]}\end{array}$ & $\begin{array}{l}1749.73 \\
{[63.2]}\end{array}$ & $\begin{array}{l}0.016 \\
{[0.0125]}\end{array}$ & 306.34 [174.86] & $53.92[18.08]$ & 218.96 [116.66] & $0.0126[0.012]$ \\
\hline
\end{tabular}




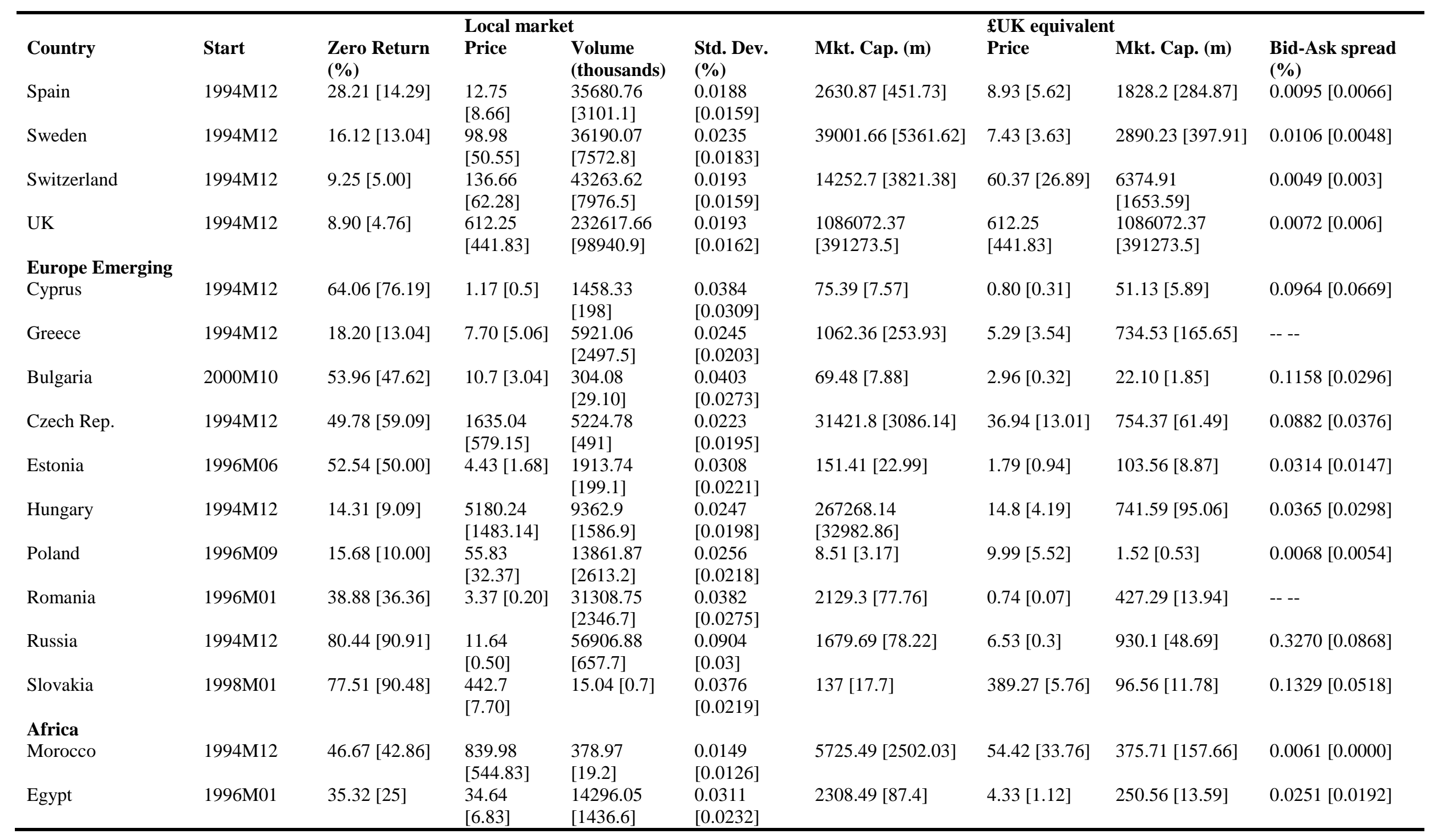




\begin{tabular}{|c|c|c|c|c|c|c|c|c|c|}
\hline \multirow[b]{2}{*}{ Country } & \multirow[b]{2}{*}{ Start } & \multirow[b]{2}{*}{$\begin{array}{l}\text { Zero Return } \\
(\%)\end{array}$} & \multicolumn{2}{|c|}{ Local market } & \multirow[b]{2}{*}{$\begin{array}{l}\text { Std. Dev. } \\
(\%)\end{array}$} & \multirow[b]{2}{*}{ Mkt. Cap. (m) } & \multicolumn{2}{|c|}{ £UK equivalent } & \multirow[b]{2}{*}{$\begin{array}{l}\text { Bid-Ask spread } \\
(\%)\end{array}$} \\
\hline & & & Price & $\begin{array}{l}\text { Volume } \\
\text { (thousands) }\end{array}$ & & & Price & Mkt. Cap. (m) & \\
\hline South Africa & 1994M12 & $16.82[9.52]$ & $\begin{array}{l}62.9 \\
{[30.44]}\end{array}$ & $\begin{array}{l}40466.08 \\
{[19627]}\end{array}$ & $\begin{array}{l}0.0219 \\
{[0.0183]}\end{array}$ & 35025 [13125.28] & $5.44[3.34]$ & $\begin{array}{l}2837.43 \\
{[1009.92]}\end{array}$ & $0.0105[0.0064]$ \\
\hline \multicolumn{10}{|l|}{ North America } \\
\hline Canada & 1994M12 & $15.28[9.52]$ & $\begin{array}{l}17.83 \\
{[10.92]}\end{array}$ & $\begin{array}{l}11420.29 \\
{[2710.9]}\end{array}$ & $\begin{array}{l}0.0275 \\
{[0.0186]}\end{array}$ & $3052.55[579.84]$ & $8.15[4.64]$ & $1419.07[247]$ & $0.0061[0.0046]$ \\
\hline United States & 1994M12 & $11.44[4.76]$ & $\begin{array}{l}36.27 \\
{[29.53]}\end{array}$ & $\begin{array}{l}89424.57 \\
{[38350.05]}\end{array}$ & $\begin{array}{l}0.0224 \\
{[0.0168]}\end{array}$ & $26319.79[7287.91]$ & $21.67[17.92]$ & $\begin{array}{l}15650.34 \\
{[4628.67]}\end{array}$ & $0.0016[0.0012]$ \\
\hline \multicolumn{10}{|l|}{ Australasia } \\
\hline Australia & 1994M12 & $15.32[9.52]$ & $8.00[4.16]$ & $\begin{array}{l}59547.76 \\
{[28767.2]}\end{array}$ & $\begin{array}{l}0.0197 \\
{[0.0153]}\end{array}$ & $6454.47[2004.41]$ & $3.33[1.70]$ & $2675.02[724.07]$ & $0.0092[0.0062]$ \\
\hline New Zealand & 1994M12 & $35.59[28.57]$ & $3.38[2.35]$ & $\begin{array}{l}11504.29 \\
{[3534.4]}\end{array}$ & $\begin{array}{l}0.0162 \\
{[0.0133]}\end{array}$ & $1264.6[313.1]$ & $1.23[0.89]$ & $463.51[96.22]$ & $0.0129[0.0108]$ \\
\hline \multicolumn{10}{|l|}{ Latin America } \\
\hline Argentina & 1994M12 & $74.64[100.00]$ & $\begin{array}{l}834.88 \\
{[2.05]}\end{array}$ & $3144.8[340]$ & $\begin{array}{l}0.0278 \\
{[0.0219]}\end{array}$ & $3314.5[24.98]$ & $275.2[0.61]$ & $766.34[12.32]$ & $0.0990[0.0296]$ \\
\hline Brazil & 1994M12 & $25.44[13.64]$ & $\begin{array}{l}15.66 \\
{[5.26]}\end{array}$ & $\begin{array}{l}14586610.82 \\
{[11112.5]}\end{array}$ & $\begin{array}{l}0.0301 \\
{[0.0243]}\end{array}$ & $4850.06[541.63]$ & $4.97[1.81]$ & $1488.85[158.2]$ & $0.0266[0.0124]$ \\
\hline Chile & 1994M12 & $62.23[72.73]$ & $\begin{array}{l}213105.07 \\
{[294.86]}\end{array}$ & $\begin{array}{l}60301.44 \\
{[931.1]}\end{array}$ & $\begin{array}{l}0.0168 \\
{[0.0133]}\end{array}$ & $\begin{array}{l}354685.71 \\
{[61655.46]}\end{array}$ & 248.69 [0.37] & 386.16 [78.48] & $0.1251[0.0664]$ \\
\hline Colombia & 1994M12 & $69.23[82.61]$ & $\begin{array}{l}5130.05 \\
{[2069.26]}\end{array}$ & $\begin{array}{l}110949.87 \\
{[861.8]}\end{array}$ & $\begin{array}{l}0.0331 \\
{[0.0163]}\end{array}$ & $\begin{array}{l}1154355.62 \\
{[217336.63]}\end{array}$ & $1.55[1.02]$ & 330.69 [96.89] & $0.1012[0.0205]$ \\
\hline Jamaica & 1994M12 & $67.66[68.18]$ & $13.1[4.36]$ & $\begin{array}{l}4334.16 \\
{[780.84]}\end{array}$ & $\begin{array}{l}0.0442 \\
{[0.0235]}\end{array}$ & $5450.06[1476.66]$ & $0.13[0.06]$ & 53.66 [18.99] & $0.125[0.0549]$ \\
\hline Mexico & 1994M12 & 24.07 [13.04] & $\begin{array}{l}19.53 \\
{[10.02]}\end{array}$ & $\begin{array}{l}91601.75 \\
{[14518.6]}\end{array}$ & $\begin{array}{l}0.0236 \\
{[0.0198]}\end{array}$ & $19444.72[5352.81]$ & $1.16[0.69]$ & $1055.16[334.4]$ & $0.0311[0.0148]$ \\
\hline Peru & 1994M12 & $78.48[95.24]$ & $\begin{array}{l}2112.56 \\
{[1.04]}\end{array}$ & $\begin{array}{l}3025.97 \\
{[213.2]}\end{array}$ & $\begin{array}{l}0.0451 \\
{[0.0218]}\end{array}$ & $65361.12[28.32]$ & $520.9[0.22]$ & $17859.35[5.42]$ & $0.2746[0.0874]$ \\
\hline Venezuela & 1994M12 & $84.65[100.00]$ & $\begin{array}{l}299.94 \\
{[0.33]}\end{array}$ & $\begin{array}{l}7239.52 \\
{[115.5]}\end{array}$ & $\begin{array}{l}0.0434 \\
{[0.0265]}\end{array}$ & $26771.6[46]$ & $76.87[0.20]$ & 8384.93 [15.01] & -- -- \\
\hline $\begin{array}{l}\text { Asia Developed } \\
\text { Japan }\end{array}$ & 1994M12 & $10.02[9.09]$ & $\begin{array}{l}40141.48 \\
{[1930.25]}\end{array}$ & $\begin{array}{l}121.87 \\
{[46.07]}\end{array}$ & $\begin{array}{l}0.023 \\
{[0.0212]}\end{array}$ & 20446.51 [993.24] & $\begin{array}{l}209.91 \\
{[10.66]}\end{array}$ & 104.23 [5.39] & $0.1650[0.0979]$ \\
\hline Singapore & 1994M12 & $40.57[38.1]$ & $1.42[0.34]$ & $\begin{array}{l}27842.9 \\
{[4024.5]}\end{array}$ & $\begin{array}{l}0.036 \\
{[0.0273]}\end{array}$ & 697.77 [86.48] & $0.58[0.12]$ & $289.73[30.44]$ & $0.0534[0.0275]$ \\
\hline
\end{tabular}




\begin{tabular}{|c|c|c|c|c|c|c|c|c|c|}
\hline \multirow[b]{2}{*}{ Country } & \multirow[b]{2}{*}{ Start } & \multirow[b]{2}{*}{$\begin{array}{l}\text { Zero Return } \\
(\%)\end{array}$} & \multicolumn{2}{|c|}{ Local market } & \multirow[b]{2}{*}{$\begin{array}{l}\text { Std. Dev. } \\
(\%)\end{array}$} & \multirow[b]{2}{*}{ Mkt. Cap. (m) } & \multicolumn{2}{|c|}{$£$ EK equivalent } & \multirow[b]{2}{*}{$\begin{array}{l}\text { Bid-Ask spread } \\
(\%)\end{array}$} \\
\hline & & & Price & $\begin{array}{l}\text { Volume } \\
\text { (thousands) }\end{array}$ & & & Price & Mkt. Cap. (m) & \\
\hline \multicolumn{10}{|l|}{ Asia Emerging } \\
\hline Bangladesh & 1994M12 & $49.67[43.48]$ & $\begin{array}{l}214.82 \\
{[87.75]}\end{array}$ & $\begin{array}{l}659.26 \\
{[18.80]}\end{array}$ & $\begin{array}{l}0.0305 \\
{[0.0204]}\end{array}$ & $\begin{array}{l}807262.89 \\
{[80180.36]}\end{array}$ & $2.39[0.98]$ & 7347.38 [916.09] & --- \\
\hline China Shenzen & 1994M12 & $16.81[9.09]$ & $6.61[4.60]$ & $\begin{array}{l}182985.16 \\
{[77766.1]}\end{array}$ & $\begin{array}{l}0.0265 \\
{[0.0218]}\end{array}$ & 4901.64 [2071.19] & $0.49[0.33]$ & $367.37[154.08]$ & $0.0058[0.0017]$ \\
\hline China Shanghai & 1994M12 & $15.38[9.09]$ & $7.13[4.92]$ & $\begin{array}{l}228292.23 \\
{[72542.8]}\end{array}$ & $\begin{array}{l}0.0263 \\
{[0.0223]}\end{array}$ & $15952.13[2250.52]$ & $0.53[0.35]$ & $1185.95[154.32]$ & $0.0025[0.0018]$ \\
\hline Hong Kong & 1994M12 & 20.27 [17.39] & $\begin{array}{l}13.66 \\
{[5.16]}\end{array}$ & $\begin{array}{l}241630.95 \\
{[69572.3]}\end{array}$ & $\begin{array}{l}0.0288 \\
{[0.0237]}\end{array}$ & 49376.34 [5310.49] & $1.04[0.4]$ & $3794.01[445.19]$ & $0.0121[0.0086]$ \\
\hline India & 1994M12 & $12.98[4.76]$ & $\begin{array}{l}269.7 \\
{[78.39]}\end{array}$ & $\begin{array}{l}22353.54 \\
{[5506.4]}\end{array}$ & $\begin{array}{l}0.0287 \\
{[0.0246]}\end{array}$ & $\begin{array}{l}95279.18 \\
{[12342.96]}\end{array}$ & $3.57[1.36]$ & $1232.4[174.37]$ & -- -- \\
\hline Indonesia & 1994M12 & $43.79[40.91]$ & $\begin{array}{l}1525.86 \\
{[369.2]}\end{array}$ & $\begin{array}{l}196184 \\
{[42013.4]}\end{array}$ & $\begin{array}{l}0.0398 \\
{[0.0285]}\end{array}$ & $\begin{array}{l}13508863.02 \\
{[232473.85]}\end{array}$ & $0.21[0.03]$ & 1101.5 [27.69] & $0.0737[0.0102]$ \\
\hline Kazakhstan & 2009M03 & $90.67[100]$ & $\begin{array}{l}14552.11 \\
{[1857.08]}\end{array}$ & $\begin{array}{l}1233.97 \\
{[137.7]}\end{array}$ & $\begin{array}{l}0.0826 \\
{[0.0591]}\end{array}$ & 70169.19 [8766.74] & $64.2[8.38]$ & $308.86[39.56]$ & $0.1345[0.0950]$ \\
\hline Malaysia & 1994M12 & $33.00[30.00]$ & $3.90[2.41]$ & $\begin{array}{l}26665.55 \\
{[7146.6]}\end{array}$ & $\begin{array}{l}0.0240 \\
{[0.0182]}\end{array}$ & 3174.79 [1095.7] & $0.70[0.39]$ & $544.62[167.14]$ & 0.0154 [0.0105] \\
\hline Pakistan & 1994M12 & $43.23[31.82]$ & $\begin{array}{l}86.62 \\
{[18.94]}\end{array}$ & $\begin{array}{l}48839.84 \\
{[728.5]}\end{array}$ & $\begin{array}{l}0.0280 \\
{[0.0234]}\end{array}$ & 11420.99 [961.11] & $0.87[0.31]$ & $110.61[15.41]$ & $0.4615[0.0440]$ \\
\hline Philippines & 1994M12 & 67.04 [69.57] & $\begin{array}{l}30.43 \\
{[1.46]}\end{array}$ & $\begin{array}{l}200120.83 \\
{[3985.9]}\end{array}$ & $\begin{array}{l}0.0575 \\
{[0.0305]}\end{array}$ & 22770.46 [844.27] & $0.42[0.02]$ & 318.78 [11.02] & 0.1496 [0.0682] \\
\hline South Korea & 1994M12 & $13.86[9.52]$ & $\begin{array}{l}57331.39 \\
{[13526.19]}\end{array}$ & $\begin{array}{l}10126.69 \\
{[3677.2]}\end{array}$ & $\begin{array}{l}0.0328 \\
{[0.0269]}\end{array}$ & $\begin{array}{l}2368313.65 \\
{[206692.76]}\end{array}$ & $35.30[8.14]$ & 1393.68 [114.17] & 0.0066 [0.0043] \\
\hline Sri Lanka & 1994M12 & 48.83 [42.86] & $\begin{array}{l}106.53 \\
{[31.44]}\end{array}$ & $\begin{array}{l}3345.32 \\
{[604.40]}\end{array}$ & $\begin{array}{l}0.0232 \\
{[0.0175]}\end{array}$ & 6964.17 [1322.34] & $0.71[0.27]$ & 37.95 [10.09] & $0.025[0.0184]$ \\
\hline Taiwan & 1994M12 & $15.74[13.04]$ & $\begin{array}{l}34.25 \\
{[22.18]}\end{array}$ & $\begin{array}{l}312421.86 \\
{[148541.60]}\end{array}$ & $\begin{array}{l}0.0243 \\
{[0.0224]}\end{array}$ & 61650.5 [19667.87] & $0.63[0.42]$ & 1107.46 [392.57] & 0.0029 [0.0024] \\
\hline Thailand & 1994M12 & 30.12 [23.81] & $\begin{array}{l}26.72 \\
{[10.56]}\end{array}$ & $\begin{array}{l}248308.32 \\
{[42030.10]}\end{array}$ & $\begin{array}{l}0.0312 \\
{[0.0241]}\end{array}$ & 24404.83 [2283.63] & $0.49[0.17]$ & $408.55[55.53]$ & $0.0105[0.0074]$ \\
\hline Vietnam & 2009M03 & 17.15 [14.29] & $\begin{array}{l}21683.6 \\
{[16291.93]}\end{array}$ & $\begin{array}{l}3804.56 \\
{[1161.40]}\end{array}$ & $\begin{array}{l}0.0319 \\
{[0.0337]}\end{array}$ & $\begin{array}{l}988143.6 \\
{[200637.5]}\end{array}$ & $0.82[0.62]$ & $37.56[7.66]$ & $0.0195[0.0177]$ \\
\hline $\begin{array}{l}\text { Middle East } \\
\text { Abu Dhabi }\end{array}$ & 2007M11 & 46.07 [33.33] & $8.82[4.58]$ & $\begin{array}{l}65378.40 \\
{[4070.8]}\end{array}$ & $\begin{array}{l}0.0337 \\
{[0.0301]}\end{array}$ & $5359.87[1782.95]$ & $1.41[0.65]$ & 811.38 [270.92] & $0.103[0.0698]$ \\
\hline
\end{tabular}




\begin{tabular}{|c|c|c|c|c|c|c|c|c|c|}
\hline \multirow[b]{2}{*}{ Country } & \multirow[b]{2}{*}{ Start } & \multirow[b]{2}{*}{$\begin{array}{l}\text { Zero Return } \\
(\%)\end{array}$} & \multicolumn{2}{|c|}{ Local market } & \multirow[b]{2}{*}{$\begin{array}{l}\text { Std. Dev. } \\
(\%)\end{array}$} & \multirow[b]{2}{*}{ Mkt. Cap. (m) } & \multicolumn{2}{|c|}{ £UK equivalent } & \multirow[b]{2}{*}{$\begin{array}{l}\text { Bid-Ask spread } \\
(\%)\end{array}$} \\
\hline & & & Price & $\begin{array}{l}\text { Volume } \\
\text { (thousands) }\end{array}$ & & & Price & Mkt. Cap. (m) & \\
\hline Dubai & 2007M12 & $82.18[95.65]$ & $\begin{array}{l}27.18 \\
{[6.64]}\end{array}$ & $\begin{array}{l}107541.53 \\
{[3240.6]}\end{array}$ & $\begin{array}{l}0.0277 \\
{[0.0241]}\end{array}$ & 2724.58 [1152.19] & $4.10[0.89]$ & 406.5 [186.55] & $0.0461[0.0152]$ \\
\hline Israel & 1994M12 & $23.35[13.64]$ & $\begin{array}{l}424.22 \\
{[18.12]}\end{array}$ & $\begin{array}{l}19054.71 \\
{[572.8]}\end{array}$ & $\begin{array}{l}0.0355 \\
{[0.0197]}\end{array}$ & $32912.58[744.88]$ & $79.89[2.84]$ & 6175.16 [120.67] & 0.0168 [0.0069] \\
\hline Jordan & 2009M01 & $27.4[23.81]$ & $2.63[1.52]$ & $\begin{array}{l}4097.45 \\
{[569.4]}\end{array}$ & $\begin{array}{l}0.0236 \\
{[0.0234]}\end{array}$ & 204.98 [28.49] & $2.54[1.48]$ & $197.71[28.38]$ & $0.0342[0.0251]$ \\
\hline Kuwait & 2009M01 & $93.38[100.00]$ & $0.58[0.33]$ & $6968.8[100]$ & $\begin{array}{l}0.0469 \\
{[0.0182]}\end{array}$ & $28.16[0.00]$ & $1.08[0.71]$ & $21.76[0.00]$ & $0.0388[0.0388]$ \\
\hline Oman & 2009M01 & $49.19[50.00]$ & $0.69[0.27]$ & $\begin{array}{l}13892.73 \\
{[9317.9]}\end{array}$ & $\begin{array}{l}0.0433 \\
{[0.0424]}\end{array}$ & $135.42[58.91]$ & $1.23[0.49]$ & $240.45[104.45]$ & $0.0156[0.0099]$ \\
\hline Qatar & 2008M09 & $51.34[75.00]$ & $\begin{array}{l}86.54 \\
{[166.21]}\end{array}$ & $\begin{array}{l}3323.33 \\
{[87.70]}\end{array}$ & $\begin{array}{l}0.0306 \\
{[0.0316]}\end{array}$ & $\begin{array}{l}10218.14 \\
{[20826.22]}\end{array}$ & $12.93[23.98]$ & $1549.46[2753]$ & $0.0870[0.1687]$ \\
\hline Saudi Arabia & 2009M01 & $7.77[4.76]$ & $\begin{array}{l}31.77 \\
{[24.06]}\end{array}$ & $\begin{array}{l}42267.8 \\
{[21145.7]}\end{array}$ & $\begin{array}{l}0.0331 \\
{[0.0313]}\end{array}$ & $7374.88[1444.79]$ & $5.77[4.45]$ & $1340.36[267.31]$ & $0.0054[0.0044]$ \\
\hline Turkey & 1994M12 & 36.65 [26.09] & $2.77[1.17]$ & $\begin{array}{l}165575.86 \\
{[46907.7]}\end{array}$ & $\begin{array}{l}0.0465 \\
{[0.0346]}\end{array}$ & $1025.06[104.52]$ & $1.71[0.73]$ & $429.36[54.28]$ & $0.0083[0.0074]$ \\
\hline
\end{tabular}


Table 2 Spearman's Rank Correlations

This table presents the non-parameterized Spearman rank correlation between the Stoll (2000) market control variables and the liquidity measures on a market by market basis. In line with Stoll (2000) the natural logarithms are taken of the variables for price, market capitalisation and volume, while volatility remains untransformed and is the monthly average of daily price variance. Price is the average of daily prices over each month and is stated in local currency units. Volume is the average of the daily trading volume over each month and is stated in thousands. MV or market capitalization is measured as of 1 January for each country and is equity market value for each firm expressed in millions of local currency units. Four liquidity measurement variables are presented. Amihud is the liquidity measure of Amihud (2002), which is defined as the daily ratio of the absolute return on a day to the UK£ trading volume for that particular day averaged over the past 1 month and provides a measure of the price impact. Liu is the measure of Liu (2006) and represents a standardized turnover-adjusted number of zero returns over the prior month. Turnover is a ratio of the traded volume of shares in relation to total number of shares outstanding and is scaled by the number of trading days in the month of measurement. It provides a measure of trading frequency. The final measure is the Bid Ask spread which is the average daily relative bid ask spread over the prior 1 month, where daily relative spread is the UK£ denominated spread divided by average of Bid and Ask prices. At the end of each month for the maximum period of data availability for each country cross sectional averages for each variable are calculated over the stocks in each respective market. Likewise at the end of each month the cross sectional Spearman's rank correlation are computed and the time series average of those correlations are reported.

\begin{tabular}{|c|c|c|c|c|c|c|c|c|}
\hline & Price & Volatility & Volume & MV & Amihud & Liu & Turnover & Bid Ask Spread \\
\hline \multicolumn{9}{|c|}{ Panel 1: Results for Common Law sub-set of World universe (2009M01 - 2009M05) } \\
\hline Price & 1.00000 & & & & & & & \\
\hline Volatility & -0.35220 & 1.00000 & & & & & & \\
\hline Volume & 0.20891 & 0.08519 & 1.00000 & & & & & \\
\hline MV & 0.76001 & -0.33501 & 0.58414 & 1.00000 & & & & \\
\hline Amihud & -0.73324 & 0.27310 & -0.74805 & -0.86701 & 1.00000 & & & \\
\hline Liu & -0.32886 & -0.02350 & -0.55034 & -0.34103 & 0.52714 & 1.00000 & & \\
\hline Turnover & 0.34061 & 0.16292 & 0.74756 & 0.30684 & -0.65689 & -0.60697 & 1.00000 & \\
\hline Bid Ask Spread & -0.66828 & 0.27636 & -0.63459 & -0.70098 & 0.87382 & 0.53509 & -0.65538 & 1.00000 \\
\hline \multicolumn{9}{|c|}{ Panel 2: Results for Civil Law sub-set of World universe (2009M01 - 2009M05) } \\
\hline Price & 1.00000 & & & & & & & \\
\hline Volatility & -0.14426 & 1.00000 & & & & & & \\
\hline Volume & 0.08630 & 0.09378 & 1.00000 & & & & & \\
\hline MV & 0.76535 & -0.13239 & 0.49792 & 1.00000 & & & & \\
\hline Amihud & -0.69284 & 0.11109 & -0.69317 & -0.87111 & 1.00000 & & & \\
\hline Liu & -0.23967 & -0.12418 & -0.53026 & -0.25492 & 0.51767 & 1.00000 & & \\
\hline Turnover & 0.20606 & 0.18453 & 0.72349 & 0.27633 & -0.63670 & -0.64591 & 1.00000 & \\
\hline Bid Ask Spread & -0.38980 & 0.09714 & -0.58780 & -0.48867 & 0.66984 & 0.48993 & -0.56515 & 1.00000 \\
\hline
\end{tabular}


Table 3 Total costs on liquidity proxies and measures

This table presents the panel cross section regression results on a firm-monthly basis using total trading costs as the dependent variable. Three liquidity measurement variables are presented. Amihud is the liquidity measure of Amihud (2002), which is defined as the daily ratio of the absolute return on a day to the UK£ trading volume for that particular day averaged over the past 1 month and provides a measure of the price impact. Liu is the measure of Liu (2006) and represents a standardized turnover-adjusted number of zero returns over the prior month. Turnover is a ratio of the traded volume of shares in relation to total number of shares outstanding and is scaled by the number of trading days in the month of measurement. It provides a measure of trading frequency. The final measure is the Bid Ask spread which is the average daily relative bid ask spread over the prior 1 month, where daily relative spread is the UK£ denominated spread divided by average of Bid and Ask prices. Firm size is determined from the first day of each month. Volatility is the average daily stock return variance and price and volume measure the average price (local currency units) and trading volume over an annual trading period. Turnover, price, volume, and market capitalisation are all log scaled in line with Stoll (2000). The White (1980) t-statistics are in parentheses.

\begin{tabular}{|c|c|c|c|c|c|c|c|c|c|}
\hline Market & $\mathbf{N}$ & Intercept & Price & Volatility & Size & Amihud & Liu & Turnover & $\overline{A d j-R^{2}}$ \\
\hline \multirow{5}{*}{ Austria } & 2,798 & $0.1160(2.04)$ & $-0.0025(-0.34)$ & $0.7120(4.88)$ & $-0.0048(-1.27)$ & & & & 0.3553 \\
\hline & & $0.1170(2.09)$ & $-0.0006(-0.08)$ & $0.6651(4.77)$ & $-0.0051(-1.37)$ & 8.80E-06 (5.02) & & & 0.3655 \\
\hline & & $0.1011(1.84)$ & $0.0018(0.23)$ & $0.7423(5.08)$ & $-0.0054(-1.46)$ & & $0.0030(3.84)$ & & 0.3770 \\
\hline & & $0.2454(3.56)$ & $0.0021(0.26)$ & $0.7573(4.95)$ & $-0.0098(-2.35)$ & & & $-0.0076(-2.99)$ & 0.3599 \\
\hline & & $0.0750(1.11)$ & $0.0027(0.35)$ & $0.6895(4.87)$ & $-0.0047(-1.23)$ & 8.21E-06 (4.90) & $0.0030(3.25)$ & $0.0015(0.54)$ & 0.3856 \\
\hline \multirow[t]{5}{*}{ Belgium } & 873 & $0.0098(3.71)$ & $-0.0016(-7.63)$ & $0.0028(0.47)$ & $-4.16 \mathrm{E}-05(-0.28)$ & & & & 0.6463 \\
\hline & & $0.0058(2.49)$ & $-0.0011(-5.49)$ & $0.0083(1.51)$ & $3.98 \mathrm{E}-05(0.29)$ & $0.0256(4.65)$ & & & 0.7565 \\
\hline & & $0.0105(3.91)$ & $-0.0016(-6.96)$ & $0.0023(0.39)$ & $-9.00 \mathrm{E}-05(-0.58)$ & & $0.0001(2.30)$ & & 0.6509 \\
\hline & & $0.0299(7.57)$ & $-0.0008(-3.38)$ & $0.0188(2.69)$ & $-0.0008(-4.33)$ & & & $-0.0008(-8.47)$ & 0.6851 \\
\hline & & $0.0191(5.40)$ & $-0.0006(-2.76)$ & $0.0178(2.75)$ & $-0.0004(-2.79)$ & 0.0237 (4.38) & 8.84E-05 (1.48) & $-0.0005(-5.90)$ & 0.7748 \\
\hline \multirow[t]{5}{*}{ Denmark } & 3,916 & $0.0601(2.54)$ & $-0.0083(-4.72)$ & $0.2422(3.42)$ & $-0.0004(-0.27)$ & & & & 0.4988 \\
\hline & & $0.0491(2.11)$ & $-0.0092(-5.30)$ & $0.2173(3.21)$ & $0.0002(0.19)$ & $0.0011(1.40)$ & & & 0.5197 \\
\hline & & $0.0082(0.33)$ & $-0.0074(-4.28)$ & $0.2616(3.91)$ & $0.0014(0.98)$ & & $0.0021(9.24)$ & & 0.5570 \\
\hline & & $0.1804(9.09)$ & $-0.0012(-0.86)$ & $0.2908(4.49)$ & $-0.0051(-4.39)$ & & & $-0.0069(-6.73)$ & 0.5457 \\
\hline & & $0.1012(5.78)$ & $-0.0026(-2.04)$ & $0.2792(4.61)$ & $-0.0020(-1.76)$ & $0.0007(1.24)$ & $0.0018(9.00)$ & $-0.0052(-6.06)$ & 0.5966 \\
\hline \multirow[t]{5}{*}{ Finland } & 336 & $0.0164(0.20)$ & $-0.0074(-1.16)$ & $0.1773(1.91)$ & $0.0002(0.04)$ & & & & 0.8653 \\
\hline & & $0.0165(0.21)$ & $-0.0072(-1.24)$ & $0.1704(2.06)$ & $0.0001(0.04)$ & $0.0002(0.33)$ & & & 0.8658 \\
\hline & & $0.0173(0.22)$ & $-0.0082(-1.15)$ & $0.1773(1.81)$ & $0.0002(0.04)$ & & $0.0003(0.75)$ & & 0.8669 \\
\hline & & $0.0199(0.24)$ & $-0.0077(-1.24)$ & $0.1827(1.93)$ & $0.0004(0.09)$ & & & $-0.0009(-1.17)$ & 0.8660 \\
\hline & & $0.0190(0.23)$ & $-0.0081(-1.35)$ & $0.1773(2.07)$ & $0.0003(0.07)$ & $0.0001(0.15)$ & $0.0002(0.87)$ & $-0.0005(-0.89)$ & 0.8664 \\
\hline
\end{tabular}




\begin{tabular}{|c|c|c|c|c|c|c|c|c|c|}
\hline Market & $\mathbf{N}$ & Intercept & Price & Volatility & Size & Amihud & Liu & Turnover & $\overline{A d j}-R^{2}$ \\
\hline \multirow[t]{5}{*}{ France } & 3,375 & $0.0791(6.79)$ & $-0.0010(-1.11)$ & 0.0239 (1.09) & $-0.0031(-5.18)$ & & & & 0.4584 \\
\hline & & $0.0487(4.69)$ & $-0.0010(-1.36)$ & $0.0229(1.11)$ & $-0.0018(-3.51($ & $0.0020(3.35)$ & & & 0.6111 \\
\hline & & $0.0713(6.74)$ & $-0.0008(-0.88)$ & 0.0317 (1.38) & $-0.0029(-4.94)$ & & 0.0017 (3.90) & & 0.5112 \\
\hline & & $0.1258(9.03)$ & $0.0014(1.72)$ & $0.0941(4.30)$ & $-0.0040(-6.98)$ & & & $-0.0043(-10.04)$ & 0.5694 \\
\hline & & $0.0843(6.97)$ & 0.0007 (1.03) & 0.0757 (3.69) & $-0.0026(-4.97)$ & $0.0015(3.03)$ & $0.0008(3.15)$ & $-0.0030(-9.86)$ & 0.6783 \\
\hline \multirow[t]{5}{*}{ Germany } & 494 & $0.0184(0.13)$ & $-0.0080(-1.08)$ & $0.0132(0.56)$ & $0.0007(0.10)$ & & & & 0.9136 \\
\hline & & $0.0179(0.13)$ & $-0.0074(-0.92)$ & $0.0195(0.60)$ & $0.0006(0.09)$ & $0.0001(0.60)$ & & & 0.9158 \\
\hline & & $0.0154(0.11)$ & $-0.0083(-1.10)$ & $0.0171(0.71)$ & $0.0009(0.12)$ & & 5.01E-05 (2.04) & & 0.9137 \\
\hline & & $0.0449(0.31)$ & $-0.0064(-0.83)$ & $0.0271(0.97)$ & $-0.0005(-0.06)$ & & & $-0.0008(-1.41)$ & 0.9140 \\
\hline & & $0.0313(0.24)$ & $-0.0067(-0.84)$ & $0.0294(0.99)$ & 4.45E-05 (0.01) & $0.0001(0.56)$ & 2.98E-05 (2.01) & $-0.0004(-1.36)$ & 0.9158 \\
\hline \multirow[t]{5}{*}{ Iceland } & 228 & $0.3242(2.44)$ & $0.0049(0.60)$ & $0.4712(2.37)$ & $-0.0144(-2.16)$ & & & & 0.1641 \\
\hline & & $0.1915(2.73)$ & $0.0007(0.11)$ & $0.2049(1.38)$ & $-0.0079(-2.38)$ & $0.0341(3.50)$ & & & 0.4223 \\
\hline & & $-0.2153(-0.84)$ & $-0.0125(-0.74)$ & $0.4652(2.86)$ & $0.0108(0.79)$ & & $0.0040(2.21)$ & & 0.2866 \\
\hline & & $0.3222(2.66)$ & $0.0061(0.50)$ & $0.5040(1.58)$ & $-0.0137(-2.74)$ & & & $-0.0028(-0.23)$ & 0.1637 \\
\hline & & $-0.2171(-0.89)$ & $-0.0169(-0.94)$ & $0.0931(0.38)$ & $0.0089(0.76)$ & $0.0321(2.72)$ & $0.0031(1.68)$ & $0.0104(0.96)$ & 0.4883 \\
\hline \multirow[t]{5}{*}{ Ireland } & 2,423 & $-0.4946(-5.56)$ & $-0.0575(-8.81)$ & $0.2763(2.22)$ & $0.0291(6.14)$ & & & & 0.7224 \\
\hline & & $-0.4961(-5.66)$ & $-0.0571(-8.96)$ & $0.2714(2.23)$ & $0.0292(6.25)$ & 6.59E-06 (3.10) & & & 0.7252 \\
\hline & & $-0.4937(-5.38)$ & $-0.0497(-6.76)$ & $0.3493(2.37)$ & $0.0278(5.78)$ & & 0.0034 (4.95) & & 0.7441 \\
\hline & & $-0.3263(-3.62)$ & $-0.0499(-7.26)$ & $0.2976(2.32)$ & $0.0222(4.65)$ & & & $-0.0054(-6.64)$ & 0.7272 \\
\hline & & $-0.4525(-4.19)$ & $-0.0479(-6.39)$ & $0.3464(2.39)$ & $0.0262(4.87)$ & 5.77E-06 (3.11) & $0.0032(4.30)$ & $-0.0013(-1.32)$ & 0.7464 \\
\hline \multirow[t]{5}{*}{ Italy } & 2,976 & $0.0051(0.35)$ & $-0.0230(-5.72)$ & $0.1218(1.73)$ & $0.0023(2.16)$ & & & & 0.3847 \\
\hline & & $-0.0139(-1.00)$ & $-0.0209(-5.72)$ & $0.1073(1.61)$ & $0.0030(3.03)$ & $0.0042(4.74)$ & & & 0.4350 \\
\hline & & $0.0145(0.96)$ & $-0.0209(-5.85)$ & $0.1507(2.45)$ & $0.0014(1.37)$ & & $0.0029(1.94)$ & & 0.4212 \\
\hline & & $0.1452(3.65)$ & $-0.0113(-4.33)$ & $0.2881(2.94)$ & $-0.0039(-2.47)$ & & & $-0.0058(-3.43)$ & 0.4351 \\
\hline & & $0.1003(3.19)$ & $-0.0115(-5.36)$ & $0.2537(2.91)$ & $-0.0023(-1.85)$ & $0.0029(4.18)$ & $0.0022(1.60)$ & $-0.0042(-2.99)$ & 0.4832 \\
\hline \multirow[t]{5}{*}{ Luxembourg } & 161 & $0.0174(1.41)$ & $-0.0002(-0.27)$ & $0.3146(3.16)$ & $-0.0004(-0.74)$ & & & & 0.1083 \\
\hline & & $0.0234(1.92)$ & $-0.0002(-0.33)$ & $0.2493(2.56)$ & $-0.0007(-1.25)$ & 0.0014 (3.09) & & & 0.1410 \\
\hline & & $0.0194(1.33)$ & $-0.0001(-0.23)$ & $0.3139(3.15)$ & $-0.0005(-0.75)$ & & $1.90 \mathrm{E}-06(0.29)$ & & 0.1029 \\
\hline & & $0.0436(2.35)$ & $-0.0002(-0.34)$ & $0.3053(2.96)$ & $-0.0015(-1.89)$ & & & $-0.0009(-1.55)$ & 0.1176 \\
\hline & & $0.0328(1.72)$ & $-0.0003(-0.42)$ & $0.2531(2.49)$ & $-0.0010(-1.33)$ & $0.0013(2.11)$ & $-3.34 \mathrm{E}-06(-0.41)$ & $-0.0004(-0.55)$ & 0.1322 \\
\hline
\end{tabular}




\begin{tabular}{|c|c|c|c|c|c|c|c|c|c|}
\hline Market & $\mathbf{N}$ & Intercept & Price & Volatility & Size & Amihud & Liu & Turnover & $\overline{A d j-R^{2}}$ \\
\hline \multirow[t]{5}{*}{ Netherlands } & 5,766 & $-0.2017(-7.11)$ & $-0.0212(-7.31)$ & $0.2677(4.71)$ & $0.0133(7.52)$ & & & & 0.4311 \\
\hline & & $-0.1952(-6.91)$ & $-0.0174(-5.97)$ & $0.2477(4.86)$ & $0.0124(7.22)$ & $0.0006(2.56)$ & & & 0.4561 \\
\hline & & $-0.1501(-6.51)$ & $-0.0164(-7.05)$ & $0.2819(5.29)$ & $0.0097(7.01)$ & & $0.0032(5.12)$ & & 0.4724 \\
\hline & & $0.0286(1.20)$ & $-0.0098(-3.63)$ & $0.3864(6.08)$ & $0.0041(3.01)$ & & & $-0.0103(-7.49)$ & 0.4639 \\
\hline & & $-0.0226(-0.98)$ & $-0.0079(-2.89)$ & $0.3323(6.10)$ & $0.0046(3.36)$ & $0.0004(2.24)$ & $0.0023(4.51)$ & $-0.0061(-8.45)$ & 0.5005 \\
\hline \multirow[t]{5}{*}{ Norway } & 2,647 & $0.0385(4.60)$ & $-0.0037(-5.35)$ & $0.0622(5.96)$ & $-0.0007(-1.70)$ & & & & 0.4795 \\
\hline & & $0.0338(4.87)$ & $-0.0031(-5.74)$ & $0.0540(6.41)$ & $-0.0006(-1.85)$ & $0.0167(5.27)$ & & & 0.5500 \\
\hline & & $0.0356(3.64)$ & $-0.0031(-3.96)$ & $0.0740(5.64)$ & $-0.0007(-1.56)$ & & $0.0014(5.68)$ & & 0.5141 \\
\hline & & $0.1536(8.05)$ & $0.0012(1.48)$ & $0.1140(8.77)$ & $-0.0050(-6.38)$ & & & $-0.0045(-9.62)$ & 0.5280 \\
\hline & & $0.1216(7.22)$ & $0.0007(1.26)$ & $0.1013(9.89)$ & $-0.0039(-5.92)$ & $0.0128(5.25)$ & $0.0007(3.36)$ & $-0.0034(-8.12)$ & 0.5874 \\
\hline \multirow[t]{5}{*}{ Portugal } & 2,437 & $0.0172(0.34)$ & $-0.0084(-1.59)$ & $0.6997(3.46)$ & $3.96 \mathrm{E}-06(0.01)$ & & & & 0.3462 \\
\hline & & $0.0121(0.23)$ & $-0.0083(-1.59)$ & $0.6856(3.36)$ & $0.0002(0.08)$ & $6.71 \mathrm{E}-06(2.21)$ & & & 0.3484 \\
\hline & & $0.0068(0.13)$ & $-0.0037(-0.75)$ & $0.5756(3.15)$ & $-0.0002(-0.09)$ & & $0.0050(7.30)$ & & 0.4321 \\
\hline & & $0.2618(4.45)$ & $0.0044(0.80)$ & $0.8629(4.29)$ & $-0.0087(-2.93)$ & & & $-0.0127(-5.92)$ & 0.3816 \\
\hline & & $0.0857(1.41)$ & $-0.0001(-0.01)$ & $0.6392(3.36)$ & $-0.0030(-0.96)$ & $6.61 \mathrm{E}-07(0.26)$ & $0.0045(6.14)$ & $-0.0040(-2.59)$ & 0.4345 \\
\hline \multirow[t]{5}{*}{ Slovenia } & 522 & $0.0236(5.28)$ & $-0.0035(-5.07)$ & $0.2261(6.29)$ & 3.32E-05 (0.11) & & & & 0.6153 \\
\hline & & $0.0155(4.86)$ & $-0.0016(-2.52)$ & $0.1662(5.91)$ & $2.99 \mathrm{E}-06(0.01)$ & $0.0028(6.31)$ & & & 0.6856 \\
\hline & & $0.0238(5.25)$ & $-0.0021(-4.83)$ & $0.2381(7.40)$ & $-0.0002(-0.86)$ & & $0.0010(9.90)$ & & 0.6964 \\
\hline & & $0.0923(12.83$ & $0.0010(1.72)$ & $0.2261(7.71)$ & $-0.0035(-9.31)$ & & & $-0.0029(-12.03)$ & 0.7311 \\
\hline & & $0.0641(10.42)$ & $0.0008(1.48)$ & $0.1997(7.45)$ & $-0.0024(-8.00)$ & $0.0015(3.62)$ & $0.0005(5.92)$ & $-0.0019(-9.06)$ & 0.7686 \\
\hline \multirow[t]{5}{*}{ Spain } & 464 & $-0.1623(-0.84)$ & $0.0089(0.67)$ & $0.2872(1.67)$ & $0.0076(0.74)$ & & & & 0.6186 \\
\hline & & $-0.1628(-0.83)$ & $0.0088(0.62)$ & $0.2901(2.65)$ & $0.0077(0.73)$ & $-0.0002(-0.03)$ & & & 0.6176 \\
\hline & & $-0.1720(-0.60)$ & $0.0083(0.50)$ & $0.2926(1.50)$ & $0.0081(0.58)$ & & $0.0001(0.12)$ & & 0.6177 \\
\hline & & $-0.1618(-0.82)$ & $0.0089(0.65)$ & $0.2843(1.90)$ & $0.0075(0.60)$ & & & $0.0003(0.03)$ & 0.6176 \\
\hline & & $-0.1711(-0.63)$ & $0.0083(0.50)$ & $0.2905(2.17)$ & $0.0080(0.52)$ & $-0.0001(-0.02)$ & $0.0001(0.08)$ & $0.0004(0.04)$ & 0.6157 \\
\hline \multirow[t]{5}{*}{ Sweden } & 7,023 & $0.1213(7.22)$ & $-0.0033(-3.10)$ & $0.4363(5.84)$ & $-0.0047(-5.34)$ & & & & 0.3563 \\
\hline & & $0.0993(5.83)$ & $-0.0036(-3.46)$ & $0.2670(5.09)$ & $-0.0035(-4.35)$ & $0.0069(2.92)$ & & & 0.5123 \\
\hline & & $0.0850(6.27)$ & $-0.0013(-1.08)$ & $0.3953(6.96)$ & $-0.0037(-4.87)$ & & 0.0057 (6.28) & & 0.5019 \\
\hline & & $0.2991(10.10)$ & $0.0052(3.72)$ & $0.4513(6.98)$ & $-0.0105(-8.63)$ & & & $-0.0098(-10.61)$ & 0.4240 \\
\hline & & $0.1735(8.99)$ & $0.0021(1.89)$ & $0.3058(6.82)$ & $-0.0063(-7.34)$ & $0.0046(2.12)$ & $0.0036(4.45)$ & $-0.0049(-8.82)$ & 0.5871 \\
\hline
\end{tabular}




\begin{tabular}{|c|c|c|c|c|c|c|c|c|c|}
\hline Market & $\mathbf{N}$ & Intercept & Price & Volatility & Size & Amihud & Liu & Turnover & $\overline{A d j}-R^{2}$ \\
\hline \multirow[t]{5}{*}{ Switzerland } & 900 & $0.0084(9.76)$ & $-1.58 \mathrm{E}-05(-0.15)$ & $0.0038(1.68)$ & $-0.0003(-8.42)$ & & & & 0.5172 \\
\hline & & $0.0072(7.87)$ & $0.0001(1.17)$ & $0.0046(1.94)$ & $-0.0002(-7.79)$ & 0.0758 (4.62) & & & 0.5228 \\
\hline & & $0.0081(8.73)$ & 7.07E-06 (0.07) & $0.0048(2.10)$ & $-0.0002(-7.69)$ & & $3.24 \mathrm{E}-05(1.27)$ & & 0.5185 \\
\hline & & $0.0201(9.36)$ & $0.0005(4.97)$ & $0.0101(4.70)$ & $-0.0007(-9.74)$ & & & $-0.0004(-6.30)$ & 0.5341 \\
\hline & & $0.0178(7.44)$ & $0.0005(5.55)$ & $0.0108(5.16)$ & $-0.0006(-8.12)$ & 0.0593 (4.35) & 2.37E-05 (1.10) & $-0.0004(-5.34)$ & 0.5373 \\
\hline \multirow[t]{5}{*}{ UK } & 6,714 & $0.0231(2.84)$ & $-0.0050(-8.95)$ & $-0.0439(-6.37)$ & 0.0004 (1.19) & & & & 0.4262 \\
\hline & & $0.0216(2.64)$ & $-0.0050(-8.86)$ & $-0.0441(-6.47)$ & 0.0005 (1.33) & $0.7650(1.84)$ & & & 0.4321 \\
\hline & & $0.0231(2.85)$ & $-0.0050(-8.96)$ & $-0.0441(-6.37)$ & $0.0004(1.21)$ & & $-3.87 \mathrm{E}-05(-0.36)$ & & 0.4262 \\
\hline & & $0.0531(5.60)$ & $-0.0039(-7.27)$ & $-0.0371(-5.27)$ & $-0.0005(-1.23)$ & & & $-0.0012(-7.53)$ & 0.4379 \\
\hline & & $0.0504(5.07)$ & $-0.0039(-7.29)$ & $-0.0383(-5.52)$ & $-0.0004(-0.98)$ & $0.6569(1.72)$ & $-0.0001(-0.95)$ & $-0.0011(-6.86)$ & 0.4425 \\
\hline \multirow[t]{5}{*}{ Cyprus } & 4,450 & $-0.3897(-7.97)$ & $-0.0508(-7.21)$ & $0.8289(8.73)$ & $0.0228(9.05)$ & & & & 0.5405 \\
\hline & & $-0.3906(-7.94)$ & $-0.0514(-7.31)$ & $0.7916(7.63)$ & $0.0229(9.06)$ & $3.05 \mathrm{E}-07$ (1.26) & & & 0.5423 \\
\hline & & $-0.3455(-7.80)$ & $-0.0397(-6.51)$ & $0.8756(9.34)$ & $0.0188(8.34)$ & & $0.0036(9.07)$ & & 0.5788 \\
\hline & & $-0.1382(-2.70)$ & $-0.0330(-4.93)$ & $0.8680(9.19)$ & $0.0123(4.72)$ & & & $-0.0096(-8.32)$ & 0.5534 \\
\hline & & $-0.3295(-6.28)$ & $-0.0389(-6.35)$ & $0.8690(8.38)$ & $0.0182(7.26)$ & $6.49 \mathrm{E}-08(0.30)$ & $0.0035(7.44)$ & $-0.0006(-0.59)$ & 0.5788 \\
\hline \multirow[t]{5}{*}{ Bulgaria } & 682 & $-0.0075(-0.36)$ & $-0.0233(-3.64)$ & 0.3905 (1.82) & $0.0076(2.52)$ & & & & 0.3460 \\
\hline & & $-0.0032(-0.13)$ & $-0.0219(-3.42)$ & $0.5483(1.61)$ & $0.0066(1.85)$ & $-2.91 \mathrm{E}-08(-0.71)$ & & & 0.3481 \\
\hline & & $-0.0174(-0.91)$ & $-0.0140(-2.45)$ & $0.3278(1.70)$ & $0.0051(1.87)$ & & 0.0037 (5.85) & & 0.3855 \\
\hline & & $0.0632(1.33)$ & $-0.0170(-3.44)$ & $0.3815(1.76)$ & $0.0024(0.63)$ & & & $-0.0041(-1.86)$ & 0.3486 \\
\hline & & $-0.0675(-1.14)$ & $-0.0162(-2.67)$ & $0.4977(1.62)$ & $0.0076(1.55)$ & $-3.14 \mathrm{E}-08(-0.91)$ & $0.0042(4.92)$ & $0.0031(1.12)$ & 0.3890 \\
\hline \multirow[t]{5}{*}{ Czech Rep. } & 402 & $-7.7994(-1.34)$ & $-0.4496(-1.32)$ & $0.1009(0.43)$ & $0.4530(1.34)$ & & & & 0.1373 \\
\hline & & $-7.7976(-1.34)$ & $-0.4482(-1.32)$ & $0.1101(0.47)$ & $0.4526(1.34)$ & $0.0003(0.35)$ & & & 0.1351 \\
\hline & & $-7.8149(-1.35)$ & $-0.4495(-1.32)$ & $0.1156(0.45)$ & $0.4536(1.34)$ & & $0.0006(0.24)$ & & 0.1352 \\
\hline & & $-8.6992(-1.46)$ & $-0.4967(-1.43)$ & $0.3625(1.57)$ & $0.5093(1.47)$ & & & $-0.0173(-2.72)$ & 0.1439 \\
\hline & & $-8.7497(-1.45)$ & $-0.5004(-1.42)$ & $0.3592(1.52)$ & $0.5132(1.46)$ & $0.0002(0.32)$ & $-0.0015(-0.55)$ & $-0.0190(-2.77)$ & 0.1401 \\
\hline \multirow[t]{5}{*}{ Estonia } & 759 & $0.2054(3.29)$ & $-0.0140(-1.79)$ & $0.7246(2.94)$ & $-0.0098(-2.73)$ & & & & 0.3146 \\
\hline & & $0.1438(3.25)$ & $-0.0082(-1.31)$ & $0.6347(2.56)$ & $-0.0069(-2.62)$ & $0.0002(2.73)$ & & & 0.3865 \\
\hline & & $0.0740(1.35)$ & $-0.0169(-2.57)$ & $0.6161(2.96)$ & $-0.0032(-0.99)$ & & $0.0044(8.52)$ & & 0.3926 \\
\hline & & $0.3423(3.90)$ & $-0.0117(-1.68)$ & $0.7278(3.20)$ & $-0.0136(-3.18)$ & & & $-0.0108(-4.63)$ & 0.3638 \\
\hline & & $0.1496(1.84)$ & $-0.0103(-2.06)$ & $0.5978(2.77)$ & $-0.0057(-1.52)$ & $0.0001(2.04)$ & $0.0025(3.21)$ & $-0.0051(-2.02)$ & 0.4346 \\
\hline
\end{tabular}




\begin{tabular}{|c|c|c|c|c|c|c|c|c|c|}
\hline Market & $\mathbf{N}$ & Intercept & Price & Volatility & Size & Amihud & Liu & Turnover & Adj-R ${ }^{2}$ \\
\hline \multirow{5}{*}{ Hungary } & 1,218 & $0.9719(3.23)$ & $0.0597(2.84)$ & $1.5357(2.71)$ & $-0.0575(-3.11)$ & & & & 0.3418 \\
\hline & & $0.5671(1.95)$ & 0.0306 (1.59) & $1.0232(1.93)$ & $-0.0319(-1.83)$ & 0.1332 (8.53) & & & 0.5510 \\
\hline & & $0.8336(3.56)$ & $0.0555(3.04)$ & $1.4815(2.75)$ & $-0.0512(-3.39)$ & & $0.0090(3.03)$ & & 0.4050 \\
\hline & & $1.3263(3.94)$ & $0.0811(3.47)$ & $1.7970(3.31)$ & $-0.0708(-3.68)$ & & & $-0.0247(-4.73)$ & 0.4434 \\
\hline & & $0.7834(2.56)$ & $0.0460(2.12)$ & $1.2343(2.43)$ & $-0.0412(-2.30)$ & $0.1120(4.91)$ & $0.0033(1.47)$ & $-0.0141(-3.56)$ & 0.5986 \\
\hline \multirow[t]{5}{*}{ Poland } & 1,268 & 0.0147 (5.97) & $-0.0009(-1.58)$ & $0.0738(3.58)$ & $-0.0004(-1.97)$ & & & & 0.2086 \\
\hline & & $0.0142(5.93)$ & $-0.0008(-1.61)$ & $0.0748(3.67)$ & $-0.0004(-1.94)$ & 1.09E-07 (0.43) & & & 0.2109 \\
\hline & & $0.0142(5.85)$ & $-0.0008(-1.42)$ & $0.0788(3.94)$ & $-0.0005(-2.33)$ & & $0.0006(2.22)$ & & 0.2226 \\
\hline & & $0.0482(3.77)$ & $0.0012(2.01)$ & $0.1232(5.83)$ & $-0.0021(-3.55)$ & & & $-0.0021(-2.92)$ & 0.2448 \\
\hline & & $0.0468(4.16)$ & $0.0012(2.08)$ & $0.1255(6.19)$ & $-0.0021(-3.84)$ & $-3.09 \mathrm{E}-08(-0.14)$ & $0.0005(2.18)$ & $-0.0020(-3.25)$ & 0.2541 \\
\hline \multirow[t]{5}{*}{ Russia } & 5,667 & $-0.1928(-2.77)$ & $-0.0623(-4.52)$ & $0.2917(1.41)$ & $0.0165(3.90)$ & & & & 0.3296 \\
\hline & & $-0.1944(-2.81)$ & $-0.0626(-4.63)$ & $0.2861(1.41)$ & $0.0166(3.94)$ & 2.69E-06 (6.26) & & & 0.3570 \\
\hline & & $-0.1407(-1.94)$ & $-0.0551(-4.08)$ & $0.3059(1.52)$ & $0.0106(2.33)$ & & $0.0041(4.10)$ & & 0.3821 \\
\hline & & $0.6832(4.73)$ & $-0.0387(-2.75)$ & $0.3000(1.49)$ & $-0.0191(-2.82)$ & & & $-0.0352(-7.48)$ & 0.3613 \\
\hline & & $0.2919(2.58)$ & $-0.0453(-3.49)$ & $0.3020(1.54)$ & $-0.0060(-1.12)$ & 2.00E-06 (4.31) & $0.0029(3.68)$ & $-0.0180(-4.70)$ & 0.4042 \\
\hline \multirow[t]{5}{*}{ Morocco } & 1,261 & $-0.0050(-0.42)$ & $0.0134(7.23)$ & $0.3927(3.17)$ & $-0.0032(-4.68)$ & & & & 0.4856 \\
\hline & & $-0.0108(-0.93)$ & $0.0138(7.35)$ & $0.3486(3.05)$ & $-0.0030(-4.42)$ & 0.0079 (4.67) & & & 0.4995 \\
\hline & & $-0.0085(-0.70)$ & $0.0136(7.69)$ & $0.4260(3.13)$ & $-0.0033(-4.73)$ & & $0.0006(3.44)$ & & 0.5072 \\
\hline & & $0.0192(0.76)$ & $0.0145(6.70)$ & $0.4161(3.06)$ & $-0.0042(-3.65)$ & & & $-0.0008(-1.02)$ & 0.4877 \\
\hline & & $-0.0307(-1.46)$ & $0.0131(6.27)$ & $0.3744(2.87)$ & $-0.0023(-2.04)$ & $0.0066(3.99)$ & $0.0006(3.23)$ & $0.0006(0.88)$ & 0.5172 \\
\hline \multirow[t]{5}{*}{ Egypt } & 1,620 & $0.0731(1.86)$ & $-0.0130(-2.75)$ & $0.3001(2.83)$ & $-0.0012(-0.50)$ & & & & 0.2625 \\
\hline & & $0.0712(1.81)$ & $-0.0130(-2.73)$ & $0.2992(2.84)$ & $-0.0011(-0.47)$ & $0.0001(0.38)$ & & & 0.2621 \\
\hline & & $0.0524(1.45)$ & $-0.0143(-3.00)$ & $0.2966(2.66)$ & $-0.0001(-0.08)$ & & $0.0015(2.07)$ & & 0.2780 \\
\hline & & $0.1020(2.20)$ & $-0.0116(-2.34)$ & $0.3209(2.79)$ & $-0.0021(-0.84)$ & & & $-0.0015(-1.16)$ & 0.2644 \\
\hline & & $0.0641(1.56)$ & $-0.0139(-2.89)$ & $0.3043(2.61)$ & $-0.0006(-0.28)$ & $-0.0001(-0.55)$ & $0.0014(1.92)$ & $-0.0004(-0.34)$ & 0.2775 \\
\hline \multirow[t]{5}{*}{ South Africa } & 3,070 & $0.0156(1.37)$ & $-0.0044(-6.86)$ & $0.0557(3.03)$ & $0.0003(0.62)$ & & & & 0.4237 \\
\hline & & $0.0134(1.20)$ & $-0.0045(-7.02)$ & $0.0534(3.03)$ & $0.0004(0.81)$ & $0.0018(1.22)$ & & & 0.4306 \\
\hline & & $0.0128(1.18)$ & $-0.0043(-6.56)$ & $0.0749(2.95)$ & $0.0003(0.65)$ & & $0.0018(3.79)$ & & 0.5101 \\
\hline & & $0.0908(5.21)$ & $-0.0016(-1.92)$ & $0.1178(5.41)$ & $-0.0020(-2.81)$ & & & $-0.0038(-6.13)$ & 0.4722 \\
\hline & & $0.0690(4.24)$ & $-0.0022(-2.71)$ & $0.1185(4.98)$ & $-0.0014(-2.06)$ & $0.0005(0.67)$ & $0.0016(3.51)$ & $-0.0028(-6.14)$ & 0.5382 \\
\hline
\end{tabular}




\begin{tabular}{|c|c|c|c|c|c|c|c|c|c|}
\hline Market & $\mathbf{N}$ & Intercept & Price & Volatility & Size & Amihud & Liu & Turnover & Adj-R ${ }^{2}$ \\
\hline \multirow[t]{5}{*}{ Canada } & 832 & $0.0431(16.74)$ & $-0.0006(-1.51)$ & $0.0867(6.93)$ & $-0.0017(-13.50)$ & & & & 0.8801 \\
\hline & & $0.0396(28.97)$ & $-0.0006(-1.73)$ & $0.0829(7.54)$ & $-0.0015(-16.32)$ & $0.0315(1.74)$ & & & 0.8842 \\
\hline & & $0.0431(16.06)$ & $-0.0006(-1.52)$ & $0.0866(6.48)$ & $-0.0017(-12.13)$ & & $-1.94 \mathrm{E}-05(-0.11)$ & & 0.8799 \\
\hline & & $0.0537(8.93)$ & $-0.0007(-1.73)$ & $0.1030(7.88)$ & $-0.0016(-8.24)$ & & & $-0.0014(-6.64)$ & 0.8885 \\
\hline & & $0.0504(14.97)$ & $-0.0007(-1.93)$ & $0.0982(8.00)$ & $-0.0015(-9.03)$ & $0.0165(0.92)$ & $-0.0001(-0.92)$ & $-0.0012(-8.25)$ & 0.8893 \\
\hline \multirow[t]{5}{*}{ United States } & 7,658 & $0.0006(0.12)$ & $-0.0039(-8.45)$ & $0.0163(2.35)$ & $0.0006(2.25)$ & & & & 0.5667 \\
\hline & & $-0.0131(-2.28)$ & $-0.0043(-8.39)$ & $0.0170(2.57)$ & $0.0012(4.11)$ & $0.0384(2.47)$ & & & 0.5913 \\
\hline & & $0.0006(0.11)$ & $-0.0039(-8.44)$ & $0.0165(2.32)$ & $0.0006(2.25)$ & & 4.17E-05 (0.63) & & 0.5668 \\
\hline & & $0.0203(2.10)$ & $-0.0033(-6.31)$ & $0.0289(3.78)$ & $-3.60 \mathrm{E}-06(-0.01)$ & & & $-0.0008(-3.14)$ & 0.5759 \\
\hline & & $0.0032(0.36)$ & $-0.0038(-6.82)$ & $0.0270(3.34)$ & 0.0007 (1.88) & $0.0356(2.29)$ & 7.02E-05 (0.97) & $-0.0006(-2.54)$ & 0.5969 \\
\hline \multirow[t]{5}{*}{ Australia } & 8,734 & $0.1225(8.50)$ & $-0.0028(-3.31)$ & $0.3499(7.75)$ & $-0.0052(-7.31)$ & & & & 0.5391 \\
\hline & & $0.1106(7.44)$ & $-0.0019(-2.37)$ & $0.3309(8.15)$ & $-0.0048(-6.52)$ & $0.0002(2.97)$ & & & 0.5986 \\
\hline & & 0.0934 (7.89) & $-0.0021(-2.57)$ & $0.3501(9.50)$ & $-0.0041(-6.77)$ & & $0.0036(7.38)$ & & 0.6316 \\
\hline & & $0.1389(9.64)$ & $-0.0020(-2.36)$ & $0.3742(7.77)$ & $-0.0055(-7.72)$ & & & $-0.0016(-3.54)$ & 0.5422 \\
\hline & & $0.0939(8.12)$ & $-0.0013(-1.69)$ & $0.3431(9.93)$ & $-0.0040(-6.74)$ & $0.0002(2.57)$ & $0.0030(5.96)$ & $-0.0004(-1.10)$ & 0.6609 \\
\hline \multirow[t]{5}{*}{ New Zealand } & 3,705 & $-0.0306(-2.52)$ & $-0.0092(-9.26)$ & $0.2723(4.49)$ & $0.0023(3.46)$ & & & & 0.4457 \\
\hline & & $-0.0347(-3.09)$ & $-0.0093(-9.78)$ & $0.2530(4.58)$ & $0.0025(4.11)$ & 4.13E-05 (6.67) & & & 0.4728 \\
\hline & & $-0.0396(-3.75)$ & $-0.0087(-8.90)$ & $0.2698(4.66)$ & $0.0026(4.39)$ & & $0.0016(4.68)$ & & 0.4930 \\
\hline & & $0.0535(2.68)$ & $-0.0061(-6.06)$ & 0.3007 (4.92) & $-0.0006(-0.72)$ & & & $-0.0040(-8.21)$ & 0.5067 \\
\hline & & $0.0265(1.74)$ & $-0.0063(-6.84)$ & $0.2762(5.10)$ & $0.0003(0.51)$ & $3.70 \mathrm{E}-05(6.69)$ & $0.0012(4.13)$ & $-0.0032(-8.93)$ & 0.5541 \\
\hline \multirow[t]{5}{*}{ Argentina } & 5,181 & $0.1723(4.49)$ & $-0.0219(-4.60)$ & 0.6029 (4.29) & $-0.0064(-3.18)$ & & & & 0.2952 \\
\hline & & $0.1074(2.18)$ & $-0.0216(-4.01)$ & $0.4954(3.68)$ & $-0.0031(-1.19)$ & $0.0010(2.90)$ & & & 0.3376 \\
\hline & & $0.1771(4.64)$ & $-0.0194(-4.15)$ & $0.6577(4.52)$ & $-0.0071(-3.56)$ & & 0.0008 (5.09) & & 0.3103 \\
\hline & & $0.3400(7.71)$ & $-0.0158(-2.88)$ & $0.7056(4.48)$ & $-0.0128(-5.84)$ & & & $-0.0091(-4.82)$ & 0.3066 \\
\hline & & $0.1793(3.42)$ & $-0.0175(-2.99)$ & $0.5817(3.95)$ & $-0.0063(-2.39)$ & $0.0009(2.69)$ & $0.0005(3.74)$ & $-0.0034(-1.79)$ & 0.3487 \\
\hline \multirow[t]{5}{*}{ Brazil } & 7,271 & $0.0646(1.23)$ & $-0.0091(-1.75)$ & 0.2918 (1.60) & $-0.0014(-0.46)$ & & & & 0.2073 \\
\hline & & $0.0448(0.85)$ & $-0.0073(-1.37)$ & $0.2772(1.54)$ & $-0.0008(-0.25)$ & $0.000945(3.53)$ & & & 0.1976 \\
\hline & & $0.0414(0.79)$ & $-0.0074(-1.40)$ & $0.3627(2.05)$ & $-0.0009(-0.29)$ & & $0.0029(3.42)$ & & 0.2907 \\
\hline & & $0.3416(3.44)$ & $0.0038(0.52)$ & $0.4445(2.51)$ & $-0.0105(-2.25)$ & & & $-0.0147(-5.90)$ & 0.2849 \\
\hline & & $0.1448(1.29)$ & $-0.0020(-0.26)$ & $0.4107(2.33)$ & $-0.0041(-0.81)$ & $8.47 \mathrm{E}-05(0.55)$ & 0.0032 (6.27) & $-0.0062(-2.07)$ & 0.2822 \\
\hline
\end{tabular}




\begin{tabular}{|c|c|c|c|c|c|c|c|c|c|}
\hline Market & $\mathbf{N}$ & Intercept & Price & Volatility & Size & Amihud & Liu & Turnover & $\overline{A d j}-R^{2}$ \\
\hline \multirow[t]{5}{*}{ Chile } & 6,051 & $-0.1234(-1.55)$ & $-0.0034(-0.45)$ & $0.4854(1.92)$ & $0.0086(2.15)$ & & & & 0.3016 \\
\hline & & $-0.1267(-1.58)$ & $-0.0035(-0.46)$ & $0.4811(1.90)$ & $0.0088(2.17)$ & $0.0041(0.55)$ & & & 0.3015 \\
\hline & & $-0.1031(-1.32)$ & $0.0016(0.22)$ & $0.5688(2.29)$ & $0.0063(1.62)$ & & $0.0012(2.72)$ & & 0.3045 \\
\hline & & $-0.0523(-0.61)$ & $0.0007(0.09)$ & $0.5596(2.16)$ & $0.0060(1.41)$ & & & $-0.0046(-2.46)$ & 0.3027 \\
\hline & & $-0.0687(-0.78)$ & $0.0032(0.41)$ & $0.5982(2.35)$ & $0.0052(1.23)$ & $-0.0014(-0.20)$ & $0.0011(2.38)$ & $-0.0023(-1.21)$ & 0.3045 \\
\hline \multirow[t]{5}{*}{ Colombia } & 281 & $-0.2204(-1.07)$ & $0.0461(1.49)$ & $0.0170(1.21)$ & $-5.12 \mathrm{E}-14(-2.02)$ & & & & 0.0541 \\
\hline & & $-0.2834(-1.19)$ & $0.0553(1.53)$ & $-0.1187(-0.85)$ & $-5.54 \mathrm{E}-14(-2.26)$ & $1.8055(0.94)$ & & & 0.0592 \\
\hline & & $-0.4032(-1.41)$ & $0.0596(1.59)$ & $0.0164(1.06)$ & $-1.68 \mathrm{E}-14(-0.29)$ & & $0.0078(1.55)$ & & 0.0263 \\
\hline & & $-0.0536(-0.29)$ & $0.0447(1.45)$ & $0.0048(0.36)$ & $-5.44 \mathrm{E}-14(-2.13)$ & & & $-0.0667(-1.70)$ & 0.0141 \\
\hline & & $-0.2918(-1.06)$ & $0.0581(1.51)$ & $-0.0239(-0.26)$ & $-2.72 \mathrm{E}-14(-0.57)$ & $0.4547(0.36)$ & $0.0061(1.41)$ & $-0.0345(-1.65)$ & 0.0249 \\
\hline \multirow[t]{5}{*}{ Jamaica } & 1,744 & $0.2169(1.56)$ & $-0.0759(-7.71)$ & $-0.0095(-1.92)$ & $0.0021(0.31)$ & & & & 0.5410 \\
\hline & & $0.2123(1.53)$ & $-0.0761(-7.70)$ & $0.0040(0.27)$ & $0.0023(0.34)$ & $-2.12 \mathrm{E}-07(-0.95)$ & & & 0.5409 \\
\hline & & $0.4867(3.48)$ & $-0.0467(-5.59)$ & $-0.0083(-1.67)$ & $-0.0144(-2.17)$ & & $0.0042(14.26)$ & & 0.6501 \\
\hline & & $0.5718(3.84)$ & $-0.0576(-7.85)$ & $-0.0117(-2.24)$ & $-0.0119(-1.84)$ & & & $-0.0162(-3.71)$ & 0.5683 \\
\hline & & $0.5222(3.81)$ & $-0.0452(-5.88)$ & $0.0120(0.72)$ & $-0.0155(-2.46)$ & $-3.25 \mathrm{E}-07(-1.31)$ & $0.0041(13.13)$ & $-0.0023(-0.85)$ & 0.6505 \\
\hline \multirow[t]{5}{*}{ Mexico } & 3,885 & $0.1523(5.82)$ & $-0.0060(-2.17)$ & $0.1967(3.58)$ & $-0.0049(-3.59)$ & & & & 0.3973 \\
\hline & & $0.1509(5.72)$ & $-0.0056(-2.05)$ & $0.1990(3.68)$ & $-0.0050(-3.57)$ & $0.0004(2.41)$ & & & 0.4101 \\
\hline & & $0.1250(4.74)$ & $-0.0007(-0.38)$ & $0.2678(5.05)$ & $-0.0049(-3.70)$ & & $0.0044(9.91)$ & & 0.5431 \\
\hline & & $0.4163(11.12)$ & $0.0057(2.19)$ & $0.3112(6.27)$ & $-0.0143(-8.72)$ & & & $-0.0120(-12.53)$ & 0.4636 \\
\hline & & $0.2206(6.25)$ & $0.0027(1.14)$ & $0.2991(5.84)$ & $-0.0082(-5.21)$ & $0.0001(0.77)$ & 0.0038 (7.79) & $-0.0042(-4.11)$ & 0.5512 \\
\hline \multirow[t]{5}{*}{ Peru } & 772 & $0.1158(2.36)$ & $-0.0311(-2.89)$ & $1.2215(3.45)$ & $-0.0016(-0.62)$ & & & & 0.4954 \\
\hline & & $0.1257(2.37)$ & $-0.0286(-2.68)$ & $0.9972(3.04)$ & $-0.0022(-0.80)$ & $0.0011(2.14)$ & & & 0.5037 \\
\hline & & $0.1929(2.13)$ & $-0.0271(-2.41)$ & $1.2278(3.48)$ & $-0.0058(-1.21)$ & & 3.43E-05 (1.99) & & 0.4950 \\
\hline & & $0.2247(2.57)$ & $-0.0262(-2.13)$ & $1.2694(3.42)$ & $-0.0060(-1.47)$ & & & $-0.0049(-1.25)$ & 0.4964 \\
\hline & & $0.2212(1.91)$ & $-0.0238(-2.02)$ & $1.0160(2.94)$ & $-0.0073(-1.25)$ & $0.0011(2.05)$ & 3.49E-05 (1.65) & $-0.0007(-0.20)$ & 0.5027 \\
\hline \multirow[t]{5}{*}{ Japan } & 44,928 & $-0.3649(-10.72)$ & $-0.0156(-2.06)$ & $-0.0013(-0.02)$ & $0.0397(9.03)$ & & & & 0.9312 \\
\hline & & $-0.3606(-10.35)$ & $-0.0158(-2.11)$ & $0.0034(0.05)$ & $0.0397(9.01)$ & $-0.8376(-0.71)$ & & & 0.9312 \\
\hline & & $-0.3669(-10.63)$ & $-0.0153(-2.02)$ & $-0.0083(-0.14)$ & 0.0395 (8.99) & & $0.0009(2.10)$ & & 0.9312 \\
\hline & & $-0.3769(-11.24)$ & $-0.0173(-2.31)$ & $-0.0021(-0.03)$ & $0.0415(9.70)$ & & & $0.0040(4.50)$ & 0.9312 \\
\hline & & $-0.3740(-10.94)$ & $-0.0172(-2.33)$ & $-0.0036(-0.06)$ & $0.0413(9.64)$ & $-0.9405(-0.79)$ & $0.0009(2.14)$ & $0.0039(4.49)$ & 0.9312 \\
\hline
\end{tabular}




\begin{tabular}{|c|c|c|c|c|c|c|c|c|c|}
\hline Market & $\mathbf{N}$ & Intercept & Price & Volatility & Size & Amihud & Liu & Turnover & $\overline{A d j-R^{2}}$ \\
\hline \multirow{5}{*}{ Singapore } & 14,217 & $-0.2030(-3.39)$ & $-0.0616(-10.56)$ & $1.1058(17.76)$ & $0.0086(2.94)$ & & & & 0.6546 \\
\hline & & $-0.2431(-4.01)$ & $-0.0638(-10.84)$ & $1.0330(16.80)$ & $0.0107(3.62)$ & 1.32E-05 (3.09) & & & 0.6593 \\
\hline & & $-0.2410(-5.61)$ & $-0.0405(-12.83)$ & $1.0262(19.68)$ & $0.0101(4.86)$ & & $0.0080(12.81)$ & & 0.7521 \\
\hline & & $0.0969(2.13)$ & $-0.0389(-8.46)$ & 1.1637 (18.99) & $-0.0001(-0.04)$ & & & $-0.0169(-14.81)$ & 0.6854 \\
\hline & & $-0.2386(-4.31)$ & $-0.0407(-10.81)$ & 0.9865 (19.04) & $0.0106(4.42)$ & 8.70E-06 (3.15) & 0.0077 (10.82) & $-0.0015(-1.58)$ & 0.7544 \\
\hline \multirow[t]{5}{*}{ China Shenzen } & 5,958 & $-0.0052(-1.63)$ & $-0.0011(-4.76)$ & $0.0401(4.38)$ & $0.0003(2.34)$ & & & & 0.0560 \\
\hline & & $-0.0080(-2.53)$ & $-0.0010(-4.36)$ & $0.0442(4.44)$ & $0.0004(3.01)$ & $0.1333(2.64)$ & & & 0.0712 \\
\hline & & $-0.0051(-1.56)$ & $-0.0011(-4.57)$ & $0.0415(4.47)$ & $0.0003(2.17)$ & & 7.27E-05 (1.95) & & 0.0601 \\
\hline & & $-0.0016(-0.49)$ & $-0.0010(-4.31)$ & $0.0507(5.75)$ & $0.0002(1.72)$ & & & $-0.0002(-2.99)$ & 0.0578 \\
\hline & & $-0.0096(-1.75)$ & $-0.0010(-4.32)$ & $0.0422(4.08)$ & $0.0005(2.46)$ & $0.1482(2.09)$ & 8.08E-05 (2.15) & 7.48E-05 (0.40) & 0.0766 \\
\hline \multirow[t]{5}{*}{ China Shanghai } & 2,444 & $0.0381(2.56)$ & 7.64E-05 (0.12) & $0.0568(4.10)$ & $-0.0016(-2.37)$ & & & & 0.0794 \\
\hline & & $0.0361(2.40)$ & $0.0001(0.20)$ & $0.0539(3.53)$ & $-0.0015(-2.23)$ & $0.3018(0.90)$ & & & 0.0800 \\
\hline & & $0.0365(2.48)$ & $0.0001(0.18)$ & $0.0653(4.55)$ & $-0.0015(-2.36)$ & & $0.0001(1.36)$ & & 0.0823 \\
\hline & & $0.0329(2.37)$ & $0.0001(0.23)$ & $0.0551(4.14)$ & $-0.0016(-2.35)$ & & & $0.0005(1.93)$ & 0.0839 \\
\hline & & $0.0214(1.67)$ & $0.0003(0.63)$ & $0.0527(3.60)$ & $-0.0013(-2.07)$ & $0.9240(2.52)$ & $0.0001(1.30)$ & $0.0010(2.74)$ & 0.0926 \\
\hline \multirow[t]{5}{*}{ Hong Kong } & 4,144 & $0.0135(8.65)$ & $-0.0030(-6.71)$ & $0.0315(4.65)$ & $-6.87 \mathrm{E}-05(-0.91)$ & & & & 0.6612 \\
\hline & & $0.0125(7.80)$ & $-0.0027(-6.57)$ & $0.0295(4.53)$ & $-5.07 \mathrm{E}-05(-0.66)$ & $0.0129(4.53)$ & & & 0.7251 \\
\hline & & $0.0132(8.06)$ & $-0.0030(-6.52)$ & $0.0316(4.38)$ & $-7.06 \mathrm{E}-05(-0.94)$ & & $0.0002(1.60)$ & & 0.6630 \\
\hline & & $0.0552(5.50)$ & $-0.0015(-3.14)$ & $0.0486(5.12)$ & $-0.0014(-4.20)$ & & & $-0.0014(-5.03)$ & 0.6776 \\
\hline & & $0.0474(5.39)$ & $-0.0015(-3.25)$ & $0.0440(5.18)$ & $-0.0012(-3.94)$ & $0.0124(4.23)$ & $6.41 \mathrm{E}-05(0.44)$ & $-0.0012(-4.87)$ & 0.7366 \\
\hline \multirow[t]{5}{*}{ Indonesia } & 1,743 & $0.5941(3.34)$ & $-0.0426(-4.51)$ & $0.0608(0.42)$ & $-0.0096(-1.71)$ & & & & 0.2340 \\
\hline & & $0.5038(3.30)$ & $-0.0389(-4.57)$ & $-0.1764(-0.83)$ & $-0.0072(-1.42)$ & 0.7853 (3.49) & & & 0.2849 \\
\hline & & $0.5676(3.25)$ & $-0.0386(-4.22)$ & $-0.0272(-0.16)$ & $-0.0098(-1.72)$ & & $0.0030(3.08)$ & & 0.2510 \\
\hline & & $0.8960(4.08)$ & $-0.0179(-2.40)$ & $0.2525(1.73)$ & $-0.0230(-3.17)$ & & & $-0.0126(-6.32)$ & 0.2474 \\
\hline & & $0.5912(3.83)$ & $-0.0316(-4.00)$ & $-0.1169(-0.51)$ & $-0.0112(-2.35)$ & $0.7041(2.65)$ & $0.0006(0.48)$ & $-0.0035(-1.35)$ & 0.2861 \\
\hline \multirow[t]{5}{*}{ Malaysia } & 7,456 & $0.0040(0.17)$ & $-0.0155(-2.91)$ & $0.6964(2.24)$ & $0.0004(0.40)$ & & & & 0.2393 \\
\hline & & $-0.0124(-0.76)$ & $-0.0093(-5.37)$ & $0.2262(1.63)$ & $0.0014(1.64)$ & $0.0002(53.06)$ & & & 0.6893 \\
\hline & & $0.0046(0.19)$ & $-0.0130(-2.84)$ & $0.6796(2.22)$ & 9.53E-05 (0.07) & & 0.0027 (5.07) & & 0.2804 \\
\hline & & $0.1314(3.94)$ & $-0.0048(-1.07)$ & $0.7981(2.48)$ & $-0.0043(-2.82)$ & & & $-0.0056(-6.28)$ & 0.2610 \\
\hline & & $0.0495(2.54)$ & $-0.0026(-1.58)$ & $0.2700(1.93)$ & $-0.0012(-1.28)$ & 0.0002 (48.69) & $0.0019(4.55)$ & $-0.0027(-6.27)$ & 0.7225 \\
\hline
\end{tabular}




\begin{tabular}{|c|c|c|c|c|c|c|c|c|c|}
\hline Market & $\mathbf{N}$ & Intercept & Price & Volatility & Size & Amihud & Liu & Turnover & $\overline{A d j}-R^{2}$ \\
\hline \multirow[t]{5}{*}{ Pakistan } & 1,036 & $-4.8256(-3.61)$ & $-0.6765(-3.20)$ & $0.1350(0.30)$ & $0.3447(4.15)$ & & & & 0.2208 \\
\hline & & $-4.0054(-3.08)$ & $-0.5711(-2.87)$ & $-0.0078(-0.02)$ & $0.2886(3.60)$ & $0.0887(9.96)$ & & & 0.3536 \\
\hline & & $-4.1554(-3.14)$ & $-0.6290(-3.07)$ & $0.2124(0.52)$ & 0.3057 (3.79) & & 0.0049 (4.69) & & 0.3008 \\
\hline & & $0.3059(0.17)$ & $-0.3225(-1.53)$ & $0.9398(1.53)$ & $0.1021(1.05)$ & & & $-0.1625(-6.61)$ & 0.3912 \\
\hline & & $-0.2450(-0.14)$ & $-0.3271(-1.53)$ & 0.6653 (1.49) & $0.1156(1.16)$ & $0.0604(6.52)$ & $0.0005(0.49)$ & $-0.1251(-5.63)$ & 0.4502 \\
\hline \multirow[t]{5}{*}{ Philippines } & 3,169 & $0.4408(3.84)$ & $-0.0418(-7.13)$ & $0.3433(4.15)$ & $-0.0143(-2.63)$ & & & & 0.6460 \\
\hline & & $0.4378(3.86)$ & $-0.0405(-7.38)$ & $0.2390(3.05)$ & $-0.0141(-2.63)$ & 0.0005 (3.31) & & & 0.6499 \\
\hline & & $0.4532(3.95)$ & $-0.0401(-6.78)$ & $0.3408(4.16)$ & $-0.0150(-2.76)$ & & $6.86 \mathrm{E}-05(2.21)$ & & 0.6468 \\
\hline & & $0.8027(6.88)$ & $-0.0191(-3.91)$ & $0.4320(4.99)$ & $-0.0286(-5.38)$ & & & $-0.0135(-8.64)$ & 0.6576 \\
\hline & & $0.7700(6.38)$ & $-0.0195(-4.02)$ & $0.3508(4.51)$ & $-0.0275(-5.01)$ & $0.0004(2.21)$ & 4.09E-05 (1.97) & $-0.0121(-7.14)$ & 0.6594 \\
\hline \multirow{5}{*}{$\begin{array}{l}\text { South } \\
\text { Korea }\end{array}$} & 14,656 & $0.0317(11.55)$ & $-6.40 \mathrm{E}-05(-0.31)$ & $0.0229(3.64)$ & $-0.0009(-6.02$ & & & & 0.4893 \\
\hline & & 0.0279 (9.39) & $-8.16 \mathrm{E}-05(-0.41)$ & $0.0224(3.62)$ & $-0.0008(-5.08)$ & $0.9145(2.02)$ & & & 0.5162 \\
\hline & & $0.0317(9.34)$ & $-1.75 E-05(-0.08)$ & $0.0283(4.00)$ & $-0.0010(-6.11)$ & & $0.0007(5.80)$ & & 0.5262 \\
\hline & & $0.0647(17.51)$ & $0.0011(5.29)$ & $0.0650(7.63)$ & $-0.0021(-12.20)$ & & & $-0.0016(-12.32)$ & 0.5243 \\
\hline & & $0.0584(12.03)$ & $0.0010(4.64)$ & $0.0653(7.37)$ & $-0.0019(-9.79)$ & $0.6976(1.92)$ & $0.0006(5.80)$ & $-0.0014(-9.78)$ & 0.5754 \\
\hline \multirow[t]{5}{*}{ Sri Lanka } & 365 & $0.3484(7.55)$ & $0.0015(0.33)$ & $0.3748(2.86)$ & $-0.0148(-7.13)$ & & & & 0.6217 \\
\hline & & $0.3439(7.44)$ & $0.0022(0.51)$ & $0.3707(2.79)$ & $-0.0147(-7.05)$ & $0.0033(0.98)$ & & & 0.6217 \\
\hline & & $0.2342(4.17)$ & $0.0014(0.36)$ & $0.3880(2.78)$ & $-0.0101(-4.05)$ & & 0.0014 (4.57) & & 0.6502 \\
\hline & & $0.3772(7.88)$ & $0.0035(0.76)$ & $0.3954(3.23)$ & $-0.0161(-7.27)$ & & & $-0.0013(-1.92)$ & 0.6260 \\
\hline & & $0.2294(3.42)$ & $0.0010(0.24)$ & $0.3882(2.73)$ & $-0.0098(-3.29)$ & $-0.0011(-0.41)$ & 0.0015 (4.01) & $0.0001(0.11)$ & 0.6484 \\
\hline \multirow[t]{5}{*}{ Taiwan } & 2,668 & $0.0313(3.11)$ & $0.0005(1.31)$ & 0.0229 (2.99) & $-0.0012(-2.70)$ & & & & 0.1563 \\
\hline & & $0.0264(2.62)$ & $0.0005(1.25)$ & $0.0221(2.91)$ & $-0.0010(-2.26)$ & $0.2240(4.33)$ & & & 0.1620 \\
\hline & & $0.0321(3.22)$ & $0.0006(1.45)$ & $0.0255(3.22)$ & $-0.0013(-2.83)$ & & 3.62E-05 (1.87) & & 0.1576 \\
\hline & & $0.0393(3.72)$ & $0.0010(2.16)$ & $0.0353(4.56)$ & $-0.0015(-3.16)$ & & & $-0.0003(-4.00)$ & 0.1640 \\
\hline & & $0.0363(3.53)$ & $0.0010(2.12)$ & $0.0362(4.42)$ & $-0.0014(-3.00)$ & $0.1357(2.60)$ & 4.21E-05 (1.90) & $-0.0003(-3.59)$ & 0.1679 \\
\hline \multirow[t]{5}{*}{ Thailand } & 7,746 & $0.0764(3.21)$ & $-0.0031(-1.97)$ & $0.0990(2.13)$ & $-0.0026(-2.26)$ & & & & 0.0637 \\
\hline & & $0.0739(3.08)$ & $-0.0029(-1.87)$ & $0.0983(2.12)$ & $-0.0025(-2.17)$ & $0.0033(1.24)$ & & & 0.0650 \\
\hline & & $0.0727(3.00)$ & $-0.0028(-1.71)$ & $0.1003(2.16)$ & $-0.0025(-2.17)$ & & $0.0009(2.78)$ & & 0.0655 \\
\hline & & $0.1505(6.19)$ & $0.0004(0.33)$ & $0.1875(3.78)$ & $-0.0053(-4.78)$ & & & $-0.0027(-5.00)$ & 0.0687 \\
\hline & & $0.1388(5.41)$ & $0.0004(0.28)$ & $0.1790(3.65)$ & $-0.0049(-4.30)$ & $0.0022(0.90)$ & $0.0006(2.05)$ & $-0.0025(-4.27)$ & 0.0702 \\
\hline
\end{tabular}




\begin{tabular}{|c|c|c|c|c|c|c|c|c|c|}
\hline Market & $\mathbf{N}$ & Intercept & Price & Volatility & Size & Amihud & Liu & Turnover & $\overline{A d j-R^{2}}$ \\
\hline \multirow[t]{5}{*}{ Vietnam } & 265 & $0.0518(2.49)$ & $0.0040(6.24)$ & $0.1847(1.02)$ & $-0.0029(-3.60)$ & & & & 0.0458 \\
\hline & & $0.0328(2.03)$ & $0.0038(9.87)$ & $0.1863(1.16)$ & $-0.0022(-3.79)$ & $3.5233(112.56)$ & & & 0.1014 \\
\hline & & $0.0447(2.68)$ & $0.0041(7.20)$ & $0.2022(1.20)$ & $-0.0028(-3.95)$ & & 0.0009 (1.95) & & 0.0461 \\
\hline & & $0.0802(2.47)$ & $0.0033(11.38)$ & $0.4459(8.23)$ & $-0.0030(-3.50)$ & & & $-0.0032(-2.37)$ & 0.0935 \\
\hline & & $0.0649(1.85)$ & $0.0032(15.55)$ & $0.3619(7.79)$ & $-0.0026(-2.95)$ & $2.6817(5.14)$ & $-0.0008(-3.60)$ & $-0.0024(-1.65)$ & 0.1145 \\
\hline \multirow[t]{5}{*}{ Abu Dhabi } & 832 & $0.7921(1.36)$ & 0.0783 (1.87) & $0.0822(0.57)$ & $-0.0373(-1.29)$ & & & & 0.2846 \\
\hline & & $0.8060(1.44)$ & $0.0822(1.97)$ & $0.0163(0.11)$ & $-0.0382(-1.37)$ & $0.0010(7.62)$ & & & 0.3064 \\
\hline & & $0.7909(1.36)$ & $0.0795(1.91)$ & $0.0892(0.62)$ & $-0.0375(-1.30)$ & & $0.0004(0.79)$ & & 0.2845 \\
\hline & & $0.8813(1.57)$ & $0.0838(2.02)$ & $0.0837(0.58)$ & $-0.0410(-1.46)$ & & & $-0.0024(-0.70)$ & 0.2843 \\
\hline & & $0.8225(1.50)$ & 0.0831 (1.99) & $0.0160(0.11)$ & $-0.0389(-1.42)$ & $0.0010(7.09)$ & $-4.28 \mathrm{E}-05(-0.08)$ & $-0.0004(-0.12)$ & 0.3046 \\
\hline \multirow[t]{5}{*}{ Dubai } & 502 & $-0.0137(-0.31)$ & $-0.0112(-4.53)$ & $0.1964(2.41)$ & $0.0027(1.32)$ & & & & 0.8133 \\
\hline & & $-0.0205(-0.45)$ & $-0.0105(-4.04)$ & $0.2115(2.67)$ & $0.0030(1.39)$ & $0.0002(3.21)$ & & & 0.8217 \\
\hline & & $0.0072(0.16)$ & $-0.0092(-3.47)$ & $0.2067(2.97)$ & $0.0013(0.66)$ & & $0.0010(3.67)$ & & 0.8268 \\
\hline & & $0.2424(4.15)$ & $1.09 \mathrm{E}-06(0.01)$ & $0.2592(3.02)$ & $-0.0069(-2.70)$ & & & $-0.0086(-5.87)$ & 0.8313 \\
\hline & & $0.1783(3.78)$ & $-0.0017(-0.63)$ & $0.2548(3.23)$ & $-0.0048(-2.26)$ & $0.0001(1.59)$ & $0.0004(1.85)$ & $-0.0062(-4.61)$ & 0.8366 \\
\hline \multirow[t]{5}{*}{ Israel } & 4,692 & $0.3247(6.75)$ & $-0.0064(-2.14)$ & 0.0965 (1.59) & $-0.0136(-5.21)$ & & & & 0.3312 \\
\hline & & $0.2266(8.72)$ & $-0.0063(-2.85)$ & $0.0461(1.76)$ & $-0.0090(-6.34)$ & 0.0042 (5.09) & & & 0.5330 \\
\hline & & $0.2171(5.19)$ & $-0.0048(-1.74)$ & $0.1197(1.90)$ & $-0.0092(-3.96)$ & & 0.0038 (4.85) & & 0.4209 \\
\hline & & $0.3681(8.49)$ & $-0.0026(-1.01)$ & $0.1567(2.17)$ & $-0.0128(-6.19)$ & & & $-0.0103(-6.83)$ & 0.3741 \\
\hline & & $0.1921(7.41)$ & $-0.0038(-1.87)$ & $0.0900(2.44)$ & $-0.0067(-5.00)$ & 0.0037 (4.91) & $0.0022(4.16)$ & $-0.0040(-4.37)$ & 0.5776 \\
\hline \multirow[t]{5}{*}{ Jordan } & 384 & $-1.7732(-2.85)$ & $-0.0526(-1.39)$ & 0.7359 (8.17) & $0.1034(2.85)$ & & & & 0.4775 \\
\hline & & $-1.7751(-2.87)$ & $-0.0518(-1.37)$ & $0.7473(8.42)$ & $0.1034(2.87)$ & 4.16E-05 (3.80) & & & 0.4767 \\
\hline & & $-1.7971(-2.86)$ & $-0.0539(-1.41)$ & $0.7821(9.30)$ & $0.1046(2.85)$ & & $0.0004(3.11)$ & & 0.4774 \\
\hline & & $-1.7929(-2.79)$ & $-0.0517(-1.33)$ & $0.7734(22.32)$ & $0.1048(2.80)$ & & & $-0.0009(-0.74)$ & 0.4764 \\
\hline & & $-1.8028(-2.82)$ & $-0.0528(-1.35)$ & $0.7964(17.42)$ & $0.1051(2.81)$ & 1.95E-05 (2.88) & 0.0003 (1.78) & $-0.0005(-0.42)$ & 0.4741 \\
\hline \multirow[t]{5}{*}{ Kuwait } & 537 & $-0.1024(-1.96)$ & $-0.0138(-1.03)$ & $0.2293(6.59)$ & $0.0063(3.24)$ & & & & 0.6462 \\
\hline & & $-0.0854(-2.65)$ & $-0.0065(-0.79)$ & $0.2024(5.09)$ & $0.0060(4.33)$ & $0.0004(1.02)$ & & & 0.6529 \\
\hline & & $-0.1380(-5.28)$ & $-0.0067(-0.60)$ & $0.3427(32.39)$ & $0.0082(7.45)$ & & 0.0017 (3.50) & & 0.6689 \\
\hline & & $-0.0282(-1.03)$ & $-0.0036(-0.46)$ & $0.2623(8.72)$ & $0.0045(3.38)$ & & & $-0.0033(-2.00)$ & 0.6510 \\
\hline & & $-0.1374(-3.15)$ & $-0.0051(-1.04)$ & $0.3203(6.85)$ & $0.0081(4.06)$ & $0.0002(0.50)$ & $0.0016(2.55)$ & $0.0004(0.27)$ & 0.6683 \\
\hline
\end{tabular}




\begin{tabular}{|c|c|c|c|c|c|c|c|c|c|}
\hline Market & $\mathbf{N}$ & Intercept & Price & Volatility & Size & Amihud & Liu & Turnover & $\overline{A d j}-R^{2}$ \\
\hline \multirow[t]{5}{*}{ Oman } & 104 & $0.0485(0.99)$ & $-0.0086(-3.21)$ & $0.0246(0.36)$ & $-0.0025(-0.93)$ & & & & 0.4972 \\
\hline & & $0.0505(0.96)$ & $-0.0084(-2.77)$ & $0.0239(0.36)$ & $-0.0026(-0.90)$ & $3.32 \mathrm{E}-06(0.22)$ & & & 0.4903 \\
\hline & & $0.1524(4.74)$ & $0.0079(2.49)$ & $0.0104(0.72)$ & $-0.0074(-4.44)$ & & $0.0031(4.37)$ & & 0.6968 \\
\hline & & $0.4771(2.68)$ & 0.0215 (1.99) & $0.0365(0.54)$ & $-0.0225(-2.65)$ & & & $-0.0048(-2.08)$ & 0.5451 \\
\hline & & $0.2803(2.21)$ & $0.0165(2.51)$ & $0.0181(1.15)$ & $-0.0134(-2.25)$ & $-1.51 \mathrm{E}-05(-2.75)$ & $0.0030(4.53)$ & $-0.0015(-0.88)$ & 0.6988 \\
\hline \multirow[t]{5}{*}{ Qatar } & 321 & $0.4224(3.09)$ & $0.0115(1.32)$ & $-0.0446(-0.73)$ & $-0.0203(-2.64)$ & & & & 0.8907 \\
\hline & & $0.4203(2.91)$ & $0.0134(1.45)$ & $-0.0523(-0.95)$ & $-0.0206(-2.52)$ & 0.0035 (2.39) & & & 0.8964 \\
\hline & & $0.3753(2.74)$ & $0.0081(0.88)$ & $-0.0102(-0.15)$ & $-0.0178(-2.29)$ & & $0.0005(3.13)$ & & 0.8961 \\
\hline & & $0.5105(4.47)$ & $0.0190(2.81)$ & $-0.0280(-0.47)$ & $-0.0247(-3.79)$ & & & $-0.0030(-2.45)$ & 0.8942 \\
\hline & & $0.4225(3.77)$ & $0.0136(1.91)$ & $-0.0192(-0.32)$ & $-0.0205(-3.14)$ & $0.0027(2.12)$ & $0.0004(3.42)$ & $-0.0012(-1.01)$ & 0.9001 \\
\hline \multirow{5}{*}{$\begin{array}{l}\text { Saudi } \\
\text { Arabia }\end{array}$} & 439 & $-0.0250(-0.21)$ & $0.0045(0.83)$ & $-0.0109(-0.44)$ & $0.0007(0.12)$ & & & & 0.4415 \\
\hline & & $-0.0065(-0.06)$ & $0.0052(0.98)$ & $-0.0048(-0.21)$ & $-0.0002(-0.03)$ & $-0.0248(-2.49)$ & & & 0.4548 \\
\hline & & $0.0063(0.06)$ & $0.0052(1.01)$ & $0.0096(0.47)$ & $-0.0009(-0.18)$ & & $0.0004(3.34)$ & & 0.4559 \\
\hline & & $0.0069(0.08)$ & $0.0059(1.41)$ & $-0.0176(-0.63)$ & $-0.0011(-0.26)$ & & & $0.0004(0.97)$ & 0.4414 \\
\hline & & $0.0245(0.32)$ & $0.0061(1.27)$ & $0.0078(0.36)$ & $-0.0019(-0.47)$ & $-0.0188(-1.95)$ & $0.0003(2.43)$ & $0.0001(0.53)$ & 0.4611 \\
\hline \multirow[t]{5}{*}{ Turkey } & 3,444 & $0.0092(1.61)$ & $-0.0016(-4.83)$ & $-0.0001(-0.44)$ & $2.31 \mathrm{E}-05(0.07)$ & & & & 0.2427 \\
\hline & & $0.0092(1.62)$ & $-0.0016(-4.99)$ & $-0.0001(-0.46)$ & $2.32 \mathrm{E}-05(0.08)$ & $-0.0002(-1.31)$ & & & 0.2433 \\
\hline & & 0.0089 (1.59) & $-0.0016(-4.73)$ & $-0.0001(-0.35)$ & $3.18 \mathrm{E}-05(0.11)$ & & $0.0001(0.84)$ & & 0.2453 \\
\hline & & $0.0266(3.71)$ & $-0.0012(-3.31)$ & $0.0007(1.20)$ & $-0.0005(-1.73)$ & & & $-0.0006(-4.18)$ & 0.2558 \\
\hline & & $0.0282(3.88)$ & $-0.0011(-3.23)$ & $0.0008(1.34)$ & $-0.0006(-1.88)$ & $-0.0004(-2.40)$ & $0.0001(0.94)$ & $-0.0006(-4.42)$ & 0.2612 \\
\hline World & 11,166 & $0.2523(5.83)$ & $0.0003(0.14)$ & $1.1626(22.32)$ & $-0.0116(-6.24)$ & & & & 0.1537 \\
\hline Common & & $0.2498(5.73)$ & $0.0004(0.17)$ & $1.0568(15.47)$ & $-0.0114(-5.96)$ & 5.41E-05 (2.19) & & & 0.1665 \\
\hline \multirow[t]{3}{*}{ Law } & & $0.0568(1.55)$ & $0.0016(0.41)$ & $1.1955(25.22)$ & $-0.0043(-2.30)$ & & $0.0103(10.02)$ & & 0.3154 \\
\hline & & $0.4128(7.18)$ & $0.0075(2.08)$ & $1.5340(19.33)$ & $-0.0096(-6.60)$ & & & $-0.0296(-7.35)$ & 0.2748 \\
\hline & & $0.1921(3.31)$ & $0.0052(1.15)$ & $1.3286(12.37)$ & $-0.0050(-2.98)$ & $3.07 \mathrm{E}-05(1.61)$ & $0.0077(\mathbf{1 0 . 6 3})$ & $-0.0161(-4.29)$ & 0.3471 \\
\hline
\end{tabular}




\begin{tabular}{|c|c|c|c|c|c|c|c|c|c|}
\hline Market & $\mathbf{N}$ & Intercept & Price & Volatility & Size & Amihud & Liu & Turnover & $\overline{A d j}-R^{2}$ \\
\hline World Civil & 11,380 & $0.1328(18.20)$ & $-0.0049(-11.61)$ & $0.2777(1.26)$ & $-0.0038(-22.22)$ & & & & 0.0950 \\
\hline \multirow{4}{*}{ Law } & & $0.1320(17.59)$ & $-0.0049(-11.67)$ & $0.2754(1.25)$ & $-0.0037(-21.78)$ & $1.46 \mathrm{E}-08(1.02)$ & & & 0.0950 \\
\hline & & $0.1316(19.61)$ & $-0.0047(-10.82)$ & $0.2738(1.25)$ & $-0.0038(-23.97)$ & & $0.0003(2.11)$ & & 0.1100 \\
\hline & & $0.2124(75.49)$ & $-0.0037(-8.14)$ & $0.2867(1.22)$ & $-0.0030(-16.24)$ & & & $-0.0138(-10.22)$ & 0.1623 \\
\hline & & $0.2070(69.51)$ & $-0.0037(-7.91)$ & $0.2837(1.21)$ & $-0.0031(-16.48)$ & 2.00E-09 (0.16) & $0.0002(1.84)$ & $-0.0130(-9.04)$ & 0.1664 \\
\hline \multirow[t]{5}{*}{ World } & 22,546 & $0.1776(20.70)$ & $-0.0041(-7.98)$ & $0.4641(1.58)$ & $-0.0063(-45.89)$ & & & & 0.1075 \\
\hline & & $0.1782(20.59)$ & $-0.0040(-7.88)$ & $0.4659(1.58)$ & $-0.0064(-48.48)$ & $-1.47 \mathrm{E}-08(-1.12)$ & & & 0.1075 \\
\hline & & $0.1723(20.80)$ & $-0.0040(-7.41)$ & $0.4589(1.58)$ & $-0.0062(-35.59)$ & & $0.0005(2.02)$ & & 0.1226 \\
\hline & & $0.2730(45.87)$ & $-0.0029(-3.39)$ & $0.5128(1.52)$ & $-0.0046(-23.42)$ & & & $-0.0185(-8.84)$ & 0.1879 \\
\hline & & $0.2671(41.47)$ & $-0.0029(-3.31)$ & $0.5120(1.53)$ & $-0.0047(-26.87)$ & $-3.43 \mathrm{E}-08(-1.87)$ & $0.0002(1.70)$ & $-0.0176(-7.99)$ & 0.1917 \\
\hline
\end{tabular}


Table 4 Likelihood ratio tests

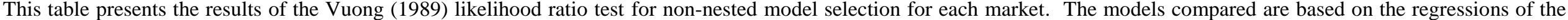

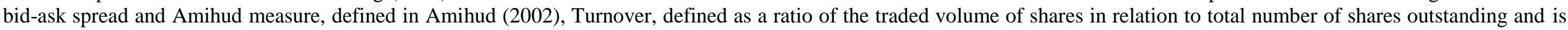

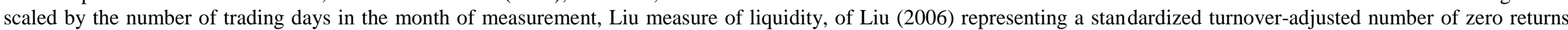

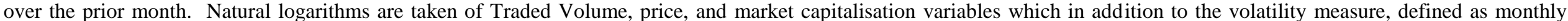

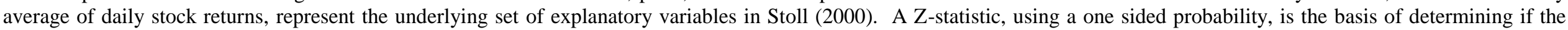

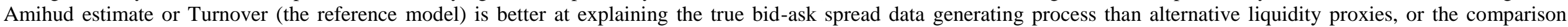

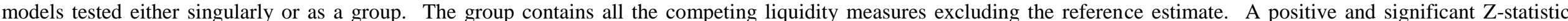

\begin{tabular}{|c|c|c|c|c|c|c|c|c|}
\hline \multirow[t]{2}{*}{ Country } & \multirow[t]{2}{*}{$\mathbf{N}$} & \multicolumn{4}{|c|}{ Liu versus } & \multicolumn{3}{|c|}{ Amihud versus } \\
\hline & & Stoll & Turnover & Amihud & Group & Stoll & Turnover & Group \\
\hline \multicolumn{9}{|c|}{ Europe Developed } \\
\hline Austria & 2,798 & -1.64 & $4.95 *$ & $3.12 *$ & $2.39 *$ & $-3.89 *$ & 0.87 & $-3.11 *$ \\
\hline Belgium & 873 & $-11.15^{*}$ & $-3.29 *$ & $-6.33^{*}$ & $-7.09 *$ & $3.12 *$ & $5.58 *$ & $5.55 *$ \\
\hline Denmark & 3,916 & 0.21 & $9.66 *$ & $7.33^{*}$ & $6.28 *$ & $-6.04 *$ & 0.87 & $-7.43 *$ \\
\hline Finland & 336 & 0.48 & $-7.18^{*}$ & $-2.24 \dagger$ & $-7.97 *$ & $2.53 *$ & -0.98 & $-2.22 \dagger$ \\
\hline France & 3,375 & -1.14 & -0.34 & $-3.12 *$ & $-4.14 *$ & $2.30 \dagger$ & $2.79 *$ & $1.98 \dagger$ \\
\hline Germany & 494 & $-18.23 *$ & $-2.65 *$ & $-5.17 *$ & $-5.11 *$ & 0.45 & $5.16 *$ & $5.04 *$ \\
\hline Iceland & 228 & $2.09 \dagger$ & $3.30 *$ & -0.46 & -0.47 & 0.72 & 1.11 & 0.36 \\
\hline Ireland & 2,423 & $-3.00 *$ & $9.93 *$ & $10.15^{*}$ & $8.44 *$ & $-11.66^{*}$ & $-7.29 *$ & $-10.15^{*}$ \\
\hline Italy & 2,976 & -1.54 & -0.52 & -1.17 & -1.84 & -0.87 & $2.08 \dagger$ & 0.18 \\
\hline Luxembourg & 161 & $-2.21 \dagger$ & -0.82 & $-2.25 \dagger$ & $-2.26 \dagger$ & -0.62 & 1.35 & 0.93 \\
\hline Netherlands & 5,766 & $4.52 *$ & $6.42 *$ & $3.47 *$ & 0.73 & -1.08 & -1.22 & $-3.78^{*}$ \\
\hline Norway & 2,647 & $-2.37 *$ & $-2.11 \dagger$ & $-2.10 \dagger$ & $-6.74 *$ & -0.35 & 0.02 & $-2.36^{*}$ \\
\hline Portugal & 2,437 & $3.25 *$ & $7.11 *$ & $8.45^{*}$ & $7.77 *$ & $-5.14 *$ & $-6.65^{*}$ & $-8.46^{*}$ \\
\hline Slovenia & 522 & $-2.68 *$ & $-3.09 *$ & $-3.66^{*}$ & $-8.16^{*}$ & 0.53 & 1.80 & 0.24 \\
\hline Spain & 464 & $2.46^{*}$ & $2.17 \dagger$ & -0.50 & -0.56 & $1.94 \dagger$ & 1.76 & 0.48 \\
\hline Sweden & 7,023 & 0.70 & $4.90 *$ & -0.23 & -0.45 & 0.68 & $2.88 *$ & 0.11 \\
\hline Switzerland & 900 & $-4.86^{*}$ & -0.39 & $-5.24 *$ & $-5.27 *$ & $-3.19 *$ & $5.11 *$ & $4.98 *$ \\
\hline UK & 6,714 & $-4.66^{*}$ & $-4.56 *$ & $-2.90 *$ & $-4.39 *$ & $-4.10 *$ & -0.32 & -0.33 \\
\hline \multicolumn{9}{|c|}{ Europe Emerging } \\
\hline Cyprus & 4,450 & $-4.56^{*}$ & $9.20 *$ & $7.40 *$ & $7.41 *$ & $-8.81 *$ & $3.02 *$ & $-7.69 *$ \\
\hline Bulgaria & 682 & 1.90 & $2.19 \dagger$ & $2.09 \dagger$ & $2.05 \dagger$ & -1.44 & 0.40 & $-2.11 \dagger$ \\
\hline Czech Rep. & 402 & 1.30 & 0.09 & 1.25 & 0.06 & -1.12 & -1.66 & -1.44 \\
\hline Estonia & 759 & -0.63 & $2.42 *$ & -0.25 & -0.48 & -0.10 & $2.94 *$ & 0.20 \\
\hline Hungary & 1,218 & -1.28 & $2.64 *$ & -1.32 & -1.32 & 1.02 & 1.58 & 1.30 \\
\hline Poland & 1,268 & $-5.51 *$ & 0.82 & -0.05 & -0.24 & $-3.89 *$ & 0.47 & -0.27 \\
\hline Russia & 5,667 & -1.45 & $3.13 *$ & 1.08 & 1.01 & $-2.33^{*}$ & $2.05 \dagger$ & -1.09 \\
\hline
\end{tabular}




\begin{tabular}{|c|c|c|c|c|c|c|c|c|}
\hline \multirow[t]{2}{*}{ Country } & \multirow[t]{2}{*}{$\mathbf{N}$} & \multicolumn{4}{|c|}{ Liu versus } & \multicolumn{3}{|c|}{ Amihud versus } \\
\hline & & Stoll & Turnover & Amihud & Group & Stoll & Turnover & Group \\
\hline \multicolumn{9}{|l|}{ Africa } \\
\hline Morocco & 1,261 & 0.51 & $5.39 *$ & $3.44 *$ & $3.12 *$ & $-3.51 *$ & $3.52 *$ & $-3.52 *$ \\
\hline Egypt & 1,620 & -0.61 & $2.00 \div$ & 1.72 & 1.68 & $-3.39 *$ & 1.84 & -1.68 \\
\hline South Africa & 3,070 & 0.74 & 0.38 & $3.48 *$ & 0.25 & $-5.94 *$ & $-5.77 *$ & $-6.90 *$ \\
\hline \multicolumn{9}{|l|}{ North America } \\
\hline Canada & 832 & $-29.24 *$ & -0.27 & $-8.96^{*}$ & $-8.85^{*}$ & $-6.80^{*}$ & $9.48 *$ & $8.92 *$ \\
\hline United States & 7,658 & $-8.71 *$ & $-3.59 *$ & $-3.49 *$ & $-5.13^{*}$ & $-4.04 *$ & $2.12 \dagger$ & $2.13 \dagger$ \\
\hline \multicolumn{9}{|l|}{ Australasia } \\
\hline Australia & 8,734 & $-2.87 *$ & $4.51 *$ & 1.18 & 1.18 & $-4.50 *$ & $3.50 *$ & -1.19 \\
\hline New Zealand & 3,705 & $-3.76^{*}$ & -0.17 & 1.76 & -1.42 & $-5.14 *$ & -1.58 & $-3.74 *$ \\
\hline \multicolumn{9}{|l|}{ Latin America } \\
\hline Argentina & 5,181 & $-2.33^{*}$ & $5.32 *$ & -1.71 & -1.85 & 0.79 & $2.57 *$ & 1.64 \\
\hline Brazil & 7,276 & $3.18 *$ & $3.82 *$ & 0.87 & 0.56 & 0.32 & 0.61 & -0.94 \\
\hline Chile & 6,051 & 0.94 & $4.61 *$ & $5.98 *$ & $4.60 *$ & $-7.25^{*}$ & $-4.96^{*}$ & $-6.04 *$ \\
\hline Colombia & 281 & 0.47 & 1.67 & 1.13 & 1.10 & -1.18 & 0.79 & -1.14 \\
\hline Jamaica & 1,744 & -0.10 & $6.07 *$ & $5.52 *$ & $6.00 *$ & $-9.08 *$ & -0.41 & $-6.32 *$ \\
\hline Mexico & 3,885 & $7.32 *$ & $9.87 *$ & $10.38 *$ & $8.14^{*}$ & $-6.68 *$ & $-2.98 *$ & $-5.84 *$ \\
\hline Peru & 772 & $-5.12 *$ & -0.51 & $-2.51 *$ & $-2.64 *$ & -1.34 & $2.36 *$ & $2.36 *$ \\
\hline \multicolumn{9}{|l|}{ Asia Developed } \\
\hline Japan & 44,928 & $-45.93 *$ & $-105.39 *$ & $2.46 *$ & -1.38 & $-45.44 *$ & $-105.37 *$ & $-5.19 *$ \\
\hline Singapore & 14,217 & $-4.52 *$ & $13.73 *$ & $11.83 *$ & $8.76^{*}$ & $-19.58 *$ & -2.23 & $-11.87 *$ \\
\hline \multicolumn{9}{|l|}{ Asia Emerging } \\
\hline China Shenzen & 5,958 & $-2.14 \dagger$ & 0.68 & -1.51 & -1.58 & 0.71 & $1.96 \dagger$ & 1.50 \\
\hline China Shanghai & 2,444 & -1.79 & -1.24 & -1.18 & $-2.02 \dagger$ & -1.56 & 0.30 & 0.17 \\
\hline Hong Kong & 4,144 & $-15.71 *$ & $-6.11 *$ & $-9.16^{*}$ & $-5.47 *$ & -0.03 & $2.93 *$ & 2.05 \\
\hline Indonesia & 1,743 & -1.32 & -0.75 & -1.42 & -1.86 & 0.90 & 1.15 & 0.98 \\
\hline Malaysia & 7,456 & -1.91 & $3.28 *$ & -1.61 & -1.71 & 1.46 & 1.75 & 1.60 \\
\hline Pakistan & 1,036 & $2.24 \dagger$ & -0.24 & -1.25 & -1.74 & 1.85 & 1.79 & 0.34 \\
\hline Philippines & 3,169 & $-10.45^{*}$ & $-11.86^{*}$ & $-4.49 *$ & $-7.16^{*}$ & $-3.08^{*}$ & 0.92 & 0.92 \\
\hline South Korea & 14,656 & $-3.43^{*}$ & -0.03 & -0.16 & $-2.13 \dagger$ & $-2.54 *$ & 0.15 & -1.64 \\
\hline Sri Lanka & 365 & 1.10 & $5.00 *$ & $5.16 *$ & $4.63 *$ & $-7.43^{*}$ & 0.59 & $-5.75^{*}$ \\
\hline Taiwan & 2,668 & $-7.97 *$ & -1.70 & $-4.84 *$ & $-4.66^{*}$ & 0.09 & $5.50 *$ & $5.28 *$ \\
\hline Thailand & 7,746 & $-3.17 *$ & 1.19 & 0.36 & 0.23 & $-3.34 *$ & 0.96 & -0.54 \\
\hline Vietnam & 265 & $-2.49 *$ & -0.68 & $-2.02 \dagger$ & -1.58 & 1.55 & $12.53 *$ & $12.85^{*}$ \\
\hline
\end{tabular}




\begin{tabular}{|c|c|c|c|c|c|c|c|c|}
\hline \multirow[t]{2}{*}{ Country } & \multirow[t]{2}{*}{$\mathbf{N}$} & \multicolumn{4}{|c|}{ Liu versus } & \multicolumn{3}{|c|}{ Amihud versus } \\
\hline & & Stoll & Turnover & Amihud & Group & Stoll & Turnover & Group \\
\hline \multicolumn{9}{|l|}{ Middle East } \\
\hline Abu Dhabi & 832 & 0.37 & $2.44 *$ & 0.36 & 0.03 & -0.18 & 1.60 & -0.36 \\
\hline Dubai & 502 & $5.03 *$ & -0.27 & $7.25 *$ & -0.99 & $-3.76^{*}$ & $-12.30 *$ & $-11.05^{*}$ \\
\hline Israel & 4,692 & $2.44 *$ & $2.75 *$ & -1.59 & $-3.11 *$ & $3.21 *$ & $3.14 *$ & 1.06 \\
\hline Jordan & 384 & -1.64 & 0.95 & 0.86 & -0.01 & $-2.18 \dagger$ & 0.92 & $-4.80 *$ \\
\hline Kuwait & 537 & $2.63 *$ & $4.13 *$ & 1.78 & 1.54 & 0.01 & $3.08 *$ & -1.80 \\
\hline Oman & 104 & $4.49 *$ & $4.16 *$ & $5.13 *$ & 4.13* & -1.29 & $-4.92 *$ & $-5.19^{*}$ \\
\hline Qatar & 321 & $4.47 *$ & -0.06 & -0.46 & $-3.22 *$ & $3.20 *$ & 0.42 & -0.46 \\
\hline Saudi Arabia & 439 & -1.64 & $-2.38 *$ & $2.33 *$ & $-2.49 *$ & $-2.85^{*}$ & $-4.50 *$ & $-6.25^{*}$ \\
\hline Turkey & 3,444 & $-5.18 *$ & -1.66 & 0.50 & -1.67 & $-5.62 *$ & $-3.13^{*}$ & $-2.02 \dagger$ \\
\hline \multicolumn{9}{|l|}{ World } \\
\hline World Common Law & 11,166 & $3.83 *$ & $2.19 \dagger$ & $4.43 *$ & $2.59 *$ & $-2.65^{*}$ & $-18.11 *$ & $-4.84 *$ \\
\hline World Civil Code & 11,380 & $-3.98 *$ & $-7.92 *$ & $1.96 \dagger$ & $-7.59 *$ & $-5.64 *$ & $-11.95^{*}$ & $-11.85^{*}$ \\
\hline World Overall & 22,546 & $-2.91 *$ & $-6.19 *$ & $2.08 \dagger$ & $-6.12 *$ & $-3.85^{*}$ & $-9.56^{*}$ & $-9.65 *$ \\
\hline
\end{tabular}

Notes: $(1) *$ Denotes significance at the $1 \%$ level

(2) $\dagger$ Denotes significance at the $5 \%$ level

(3) $\mathrm{Z}$ critical values at $90 \%, 95 \%$ and $99 \%$ confidence levels are 1.28, 1.96 and 2.33 
Table 5 Summary statistics for equally weighted monthly excess returns on 9 portfolios formed on size and illiquidity for period 2000 to 2009

This table presents the summary statistics for each of the nine size-illiquidity sorted portfolios. For each year, $t$, every stock is ranked by its market capitalisation of equity and the end of December in year t. Stocks are then classified into 3 portfolios based on market value, from the smallest to the largest. For each size portfolio, stocks are further sorted into 3 Illiquidity portfolios based on individual stocks Illiquidity ranking in ascending order. Nine size-illiquidity are so formed and rebalanced annually. The equally weighted monthly returns on portfolios are computed each month from January to the following December. Repeating this procedure for every year results in an overall sample set of 143 equally weighted monthly returns from January 2000 to June 2009. The terms B, M, S delineate Big, Medium and Small size and H, M, L delineate High, Medium and Low illiquidity terms. Additionally for each sample time period three zero cost portfolios, SMB (HML) representing long small size (high illiquidity) portfolios and short large size (low illiquidity) portfolios and LEGAL representing differences in value depending on whether stock is traded in a civil or common code legal regulatory regime. Annual rebalancing takes place annually every December.

\begin{tabular}{|c|c|c|c|c|c|c|c|c|c|}
\hline Portfolio & $\mathbf{S} / \mathbf{L}$ & $\mathbf{S} / \mathbf{M}$ & $\mathbf{S} / \mathbf{H}$ & $\mathbf{M} / \mathbf{L}$ & $\mathbf{M} / \mathbf{M}$ & $\mathbf{M} / \mathbf{H}$ & $\mathbf{B} / \mathbf{L}$ & $\mathbf{B} / \mathbf{M}$ & $\mathbf{B} / \mathbf{H}$ \\
\hline \multicolumn{10}{|c|}{ Panel A: Summary Statistics for portfolios during sample period: $01 / 2000-06 / 2009$} \\
\hline Mean & 0.01313 & 0.01539 & 0.01351 & 0.01149 & 0.01273 & 0.01375 & 0.01111 & 0.01240 & 0.01014 \\
\hline Median & 0.01889 & 0.02534 & 0.01805 & 0.01932 & 0.02080 & 0.01691 & 0.01562 & 0.02195 & 0.01941 \\
\hline Standard Deviation & 0.05185 & 0.05228 & 0.05025 & 0.04947 & 0.05188 & 0.05300 & 0.05039 & 0.05215 & 0.05006 \\
\hline Kurtosis & -0.266 & -0.481 & -0.116 & -0.692 & -0.525 & 0.004 & -0.471 & -0.713 & -0.724 \\
\hline Skewness & 4.733 & 4.143 & 4.830 & 4.026 & 4.287 & 6.265 & 4.232 & 4.236 & 4.211 \\
\hline \multicolumn{10}{|c|}{ Panel B: Average number of stocks in each of the 9 size-illiquidity portfolios sorted by region by year in period: $2000-2009$} \\
\hline Europe Developed & 97.655 & 92.566 & 88.770 & 86.425 & 81.080 & 87.212 & 108.805 & 87.212 & 93.088 \\
\hline - UK & 8.319 & 8.850 & 8.469 & 12.009 & 9.434 & 6.681 & 14.699 & 7.850 & 5.920 \\
\hline - France & 4.212 & 4.407 & 4.637 & 3.053 & 3.531 & 3.814 & 3.115 & 2.619 & 3.363 \\
\hline Europe Emerging & 34.646 & 31.310 & 47.947 & 38.770 & 27.912 & 47.770 & 30.327 & 31.000 & 42.850 \\
\hline Africa & 10.292 & 7.743 & 11.894 & 5.717 & 8.407 & 10.372 & 8.354 & 8.416 & 11.018 \\
\hline North America & 75.274 & 72.097 & 62.752 & 69.239 & 65.717 & 60.478 & 79.336 & 70.044 & 60.425 \\
\hline - United States & 54.416 & 51.929 & 47.097 & 51.035 & 47.044 & 41.442 & 55.637 & 47.389 & 47.487 \\
\hline Australasia & 16.991 & 12.319 & 13.195 & 12.274 & 10.584 & 15.354 & 13.133 & 8.965 & 14.903 \\
\hline Latin America & 61.885 & 51.062 & 72.310 & 71.867 & 54.310 & 15.354 & 65.690 & 52.540 & 73.876 \\
\hline Asia Developed & 42.566 & 53.230 & 51.142 & 46.929 & 39.035 & 33.584 & 42.867 & 57.770 & 46.106 \\
\hline - Japan & 8.602 & 10.965 & 9.699 & 9.912 & 13.150 & 7.973 & 9.071 & 8.177 & 6.920 \\
\hline Asia Emerging & 119.336 & 148.035 & 129.619 & 132.274 & 187.690 & 152.885 & 120.159 & 159.796 & 133.080 \\
\hline Middle East & 14.611 & 13.310 & 6.487 & 14.035 & 10.319 & 12.858 & 11.885 & 11.619 & 9.221 \\
\hline Overall & 473.257 & 481.673 & 484.115 & 477.531 & 485.053 & 477.619 & 480.558 & 487.363 & 484.566 \\
\hline
\end{tabular}


Table 6. Summary statistics for equally weighted monthly excess returns on 9 portfolios formed on size and illiquidity for period 2001 to 2009

\begin{tabular}{lllll}
\hline Market & Mean & $\begin{array}{l}\text { Standard } \\
\text { Deviation }\end{array}$ & Kurtosis & Skewness \\
Summary statistics & & 0.04922 & -0.770 & 4.103 \\
MARKET & 0.01280 & 0.01151 & 1.544 & 9.921 \\
SMB & -0.00088 & 0.01297 & 1.058 & 17.725 \\
HML & -0.00312 & 0.02290 & 0.146 & 3.269 \\
LEGAL & -0.00675 & & HML & LEGAL \\
Correlations & MARKET & SMB & & \\
MARKET & 1.00000 & & & \\
SMB & -0.12484 & 1.00000 & & \\
HML & -0.01932 & 0.00539 & 1.00000 & \\
LEGAL & -0.15422 & -0.08521 & -0.16638 & \\
T-Difference in Means & & & & \\
& MARKET & SMB & HML & \\
MARKET & ---- & & & \\
SMB & $2.87602 \dagger$ & --- & -- & \\
HML & $3.32364^{*}$ & 1.37199 & & \\
LEGAL & $3.82752^{*}$ & $2.43450^{* *}$ & 1.46733 & \\
\hline
\end{tabular}

Notes: $(1) *$ Denotes significance at the $1 \%$ level

(2) $\dagger$ Denotes significance at the $5 \%$ level

(3) ** Denotes significance at the $10 \%$ level

(4) t-critical values at $90 \%, 95 \%$ and $99 \%$ confidence levels are $1.65,2.58$ and 3.29 
Table 7 Summary statistics for aggregate market portfolios and equally weighted monthly excess returns on 9 portfolios formed on size and illiquidity for period 2000 to 2009

This table presents the summary descriptive statistics and correlations between the market, size, liquidity and legal regime valuation factors. For each year, t, every company is ranked by its market capitalisation of equity and the end of December in year t. Stocks are then classified into 3 portfolios based on market value, from the smallest to the largest. For each size portfolio, stocks are further sorted into 3 Illiquidity portfolios based on individual stocks Illiquidity ranking in ascending order. Nine size-illiquidity are so formed and rebalanced annually. The equally weighted monthly returns on portfolios are computed each month from January to the following December. Repeating this procedure for every year results in an overall sample set of 143 equally weighted monthly returns from January 2000 to June 2009.

\begin{tabular}{|c|c|c|c|c|}
\hline Market & Mean & $\begin{array}{l}\text { Standard } \\
\text { Deviation }\end{array}$ & Kurtosis & Skewness \\
\hline \multicolumn{5}{|c|}{ Europe Developed } \\
\hline Austria & 0.00558 & 0.04716 & -0.408 & 5.431 \\
\hline Belgium & 0.00384 & 0.05852 & -0.584 & 5.427 \\
\hline Denmark & 0.01151 & 0.06157 & -0.672 & 5.322 \\
\hline Finland & 0.01160 & 0.05897 & -0.317 & 4.349 \\
\hline France & 0.00438 & 0.07056 & -0.408 & 4.437 \\
\hline Germany & 0.00588 & 0.06757 & -0.275 & 5.769 \\
\hline Iceland & 0.00089 & 0.07295 & -0.153 & 3.969 \\
\hline Ireland & 0.00882 & 0.07299 & -0.010 & 7.341 \\
\hline Italy & 0.00062 & 0.06922 & -0.316 & 3.437 \\
\hline Luxembourg & 0.00328 & 0.07698 & -0.896 & 5.877 \\
\hline Netherlands & 0.00356 & 0.06636 & -0.457 & 3.878 \\
\hline Norway & 0.01168 & 0.09051 & -0.708 & 4.646 \\
\hline Portugal & 0.00282 & 0.05385 & -0.420 & 3.787 \\
\hline Slovenia & 0.01249 & 0.06178 & -0.650 & 4.870 \\
\hline Spain & 0.00769 & 0.04629 & -0.050 & 4.146 \\
\hline Sweden & 0.01139 & 0.07848 & -0.155 & 3.639 \\
\hline Switzerland & 0.00456 & 0.06469 & -0.138 & 4.247 \\
\hline UK & 0.00498 & 0.04937 & -0.793 & 4.339 \\
\hline \multicolumn{5}{|c|}{ Europe Emerging } \\
\hline Cyprus & -0.00448 & 0.10023 & -0.200 & 3.711 \\
\hline Greece & 0.00072 & 0.09461 & -0.054 & 3.638 \\
\hline Bulgaria & 0.03851 & 0.13893 & 1.665 & 8.954 \\
\hline Czech Rep. & 0.01590 & 0.05871 & 0.182 & 4.669 \\
\hline Estonia & 0.01226 & 0.06183 & -0.110 & 4.785 \\
\hline Hungary & 0.01161 & 0.08571 & -0.340 & 3.597 \\
\hline Poland & 0.01622 & 0.10354 & -0.017 & 3.398 \\
\hline Romania & 0.02539 & 0.12792 & 0.219 & 5.240 \\
\hline Russia & 0.07720 & 0.22955 & 4.196 & 23.492 \\
\hline Slovakia & 0.00917 & 0.06099 & -0.544 & 4.665 \\
\hline \multicolumn{5}{|l|}{ Africa } \\
\hline Morocco & 0.01049 & 0.05524 & 0.355 & 3.716 \\
\hline Egypt & 0.02562 & 0.10687 & 0.537 & 3.847 \\
\hline South Africa & 0.01346 & 0.07469 & -0.352 & 2.793 \\
\hline \multicolumn{5}{|c|}{ North America } \\
\hline Canada & 0.01933 & 0.06036 & -0.838 & 4.112 \\
\hline United States & 0.00623 & 0.04419 & 0.017 & 3.159 \\
\hline \multicolumn{5}{|l|}{ Australasia } \\
\hline Australia & 0.01386 & 0.06437 & -0.545 & 4.947 \\
\hline New Zealand & 0.00629 & 0.05500 & -0.449 & 3.960 \\
\hline \multicolumn{5}{|c|}{ Latin America } \\
\hline Argentina & -0.00283 & 0.06515 & -1.743 & 10.899 \\
\hline Brazil & 0.02563 & 0.11951 & 0.313 & 6.295 \\
\hline Chile & 0.01028 & 0.05300 & -0.283 & 3.768 \\
\hline Colombia & 0.01731 & 0.06787 & -0.193 & 3.321 \\
\hline Jamaica & 0.03798 & 0.18178 & 6.609 & 58.568 \\
\hline Mexico & 0.01138 & 0.07785 & -0.445 & 3.715 \\
\hline Peru & 0.02678 & 0.05636 & 1.476 & 6.982 \\
\hline Venezuela & 0.02338 & 0.11918 & 4.510 & 33.328 \\
\hline
\end{tabular}




\begin{tabular}{lllll}
\hline Asia Developed & & & & \\
Japan & 0.00062 & 0.05890 & 0.284 & 2.981 \\
Singapore & 0.00602 & 0.04806 & -0.261 & 3.338 \\
Asia Emerging & & & & \\
Bangladesh & 0.01619 & 0.06601 & 1.167 & 6.035 \\
China Shenzen & 0.01917 & 0.09344 & 0.264 & 3.572 \\
China Shanghai & 0.01731 & 0.09247 & 0.374 & 3.684 \\
Hong Kong & 0.01835 & 0.07927 & -0.284 & 4.092 \\
India & 0.03011 & 0.10719 & -0.107 & 3.686 \\
Indonesia & -0.01092 & 0.09967 & -0.873 & 5.126 \\
Malaysia & 0.00464 & 0.06133 & 0.014 & 3.276 \\
Pakistan & 0.02325 & 0.08404 & 0.164 & 4.036 \\
Philippines & 0.02714 & 0.11383 & 4.195 & 32.641 \\
South Korea & 0.02027 & 0.10475 & 0.470 & 3.740 \\
Sri Lanka & 0.01975 & 0.09911 & 2.591 & 16.158 \\
Taiwan & 0.00851 & 0.08731 & 0.220 & 3.211 \\
Thailand & 0.01723 & 0.09840 & 0.144 & 3.787 \\
Middle East & & & & \\
Israel & 0.01158 & 0.07796 & 0.175 & 3.527 \\
Turkey & 0.02606 & 0.22446 & 3.917 & 32.487 \\
\hline
\end{tabular}


Table 8 Time series regressions using equally weighted monthly contemporaneous market excess returns for 9 portfolios formed on size and illiquidity for period: 1996 - 2001, for all sample markets.

This table contrasts the performance of one factor CAPM with the four factor adjusted CAPM in modelling the nine size-illiquidity sorted portfolios. Stocks are sorted in ascending order on monthly basis in accordance to Amihud (2002) illiquidity measure. The size-liquidity portfolios are formed by first sorting stocks into three portfolios, Big, Medium and Small, depending on their market capitalisation and then further sorting stocks within each size portfolio into three further illiquidity portfolios, High, Medium and Low, on an annual basis. The SMB factor is formed through the difference between small and big size portfolios. Correspondingly the HML illiquidity factor portfolio is formed from the sum of the three high illiquidity portfolios less the sum of the three low illiquidity portfolios. The LEGAL factor is formed from the aggregate excess returns of civil code law markets less the aggregate excess returns of common law markets. Panel A presents parameter estimates of the capital asset pricing model, CAPM:

$r_{i t}-r_{f t}=\alpha_{i}+\beta_{i}\left(r_{m t}-r_{f t}\right)+\varepsilon_{i t}$

panel B presents parameter estimates of the three factor adjusted CAPM model:

$r_{i t}-r_{f t}=\alpha_{i}+\beta_{i}\left(r_{m t}-r_{f t}\right)+s_{i} S M B_{t}+h_{i} H M L_{t}+\varepsilon_{i t}$

and panel $\mathrm{C}$ presents parameter estimates of the four factor adjusted CAPM model:

$r_{i t}-r_{f t}=\alpha_{i}+\beta_{i}\left(r_{m t}-r_{f t}\right)+s_{i} S M B_{t}+h_{i} H M L_{t}+z_{i} L E G A L_{t}+\varepsilon_{i t}$

where $r_{i t}$ is the return of portfolio $i$ in month $t, r_{f t}$ is the one month T-bill risk free rate for month $t$, which is taken as the one month UK Gilt rate in this case. Numbers in parentheses are t-statistics.

\begin{tabular}{|c|c|c|c|c|c|c|c|c|c|}
\hline Portfolio & $\mathbf{S} / \mathbf{L}$ & $\mathbf{S} / \mathbf{M}$ & $\mathbf{S} / \mathbf{H}$ & $\mathbf{M} / \mathbf{L}$ & $\mathbf{M} / \mathbf{M}$ & $\mathbf{M} / \mathbf{H}$ & $\mathbf{B} / \mathbf{L}$ & B/M & $\mathbf{B} / \mathbf{H}$ \\
\hline \multicolumn{10}{|c|}{ Panel A: CAPM-adjusted performance } \\
\hline \multirow{2}{*}{$\hat{\alpha}(\%)$} & 0.0003 & 0.0028 & 0.0013 & -0.0011 & -0.0002 & 0.0009 & -0.0015 & -0.0009 & -0.0026 \\
\hline & $(0.22)$ & $(1.35)$ & $(0.88)$ & $(-1.34)$ & $(-0.22)$ & $(0.62)$ & $(-1.65)$ & $(-0.88)$ & $(-4.71)$ \\
\hline \multirow{2}{*}{$\hat{\beta}$} & 1.0023 & 0.9813 & 0.9537 & 0.9871 & 1.0161 & 1.0013 & 0.9906 & 1.0402 & 1.0019 \\
\hline & $(40.44)$ & $(32.55)$ & $(30.35)$ & $(50.98)$ & $(40.32)$ & $(26.06)$ & $(38.14)$ & $(66.90)$ & $(68.79)$ \\
\hline $\operatorname{Adj} R^{2}(1)$ & 0.9044 & 0.8523 & 0.8715 & 0.9641 & 0.9286 & 0.8636 & 0.9357 & 0.9637 & 0.9699 \\
\hline \multicolumn{10}{|c|}{ Panel B: Three-factor CAPM performance } \\
\hline \multirow{2}{*}{$\hat{\alpha}$} & -0.0015 & 0.0028 & 0.0037 & -0.0018 & -0.0015 & 0.0035 & -0.0030 & -0.0004 & -0.0026 \\
\hline & $(-1.54)$ & $(1.40)$ & $(2.32)$ & $(-2.32)$ & $(-1.37)$ & $(1.91)$ & $(-3.75)$ & $(-0.41)$ & $(-3.68)$ \\
\hline \multirow{2}{*}{$\hat{\beta}$} & 1.0157 & 1.0005 & 0.9698 & 0.9816 & 1.0192 & 0.9996 & 0.9695 & 1.0337 & 0.9901 \\
\hline & $(41.69)$ & $(27.89)$ & (38.58) & $(56.48)$ & $(37.28)$ & $(34.47)$ & $(59.38)$ & $(60.80)$ & $(75.49)$ \\
\hline \multirow[t]{2}{*}{$\hat{s}$} & 0.5838 & 0.6768 & 0.4247 & -0.1540 & 0.1819 & -0.2142 & -0.6570 & -0.2548 & -0.4202 \\
\hline & (1.97) & (1.32) & $(1.70)$ & $(-1.05)$ & $(0.65)$ & $(-0.98)$ & $(-3.75)$ & $(-1.83)$ & $(-5.75)$ \\
\hline \multirow{2}{*}{$\hat{h}$} & -0.7099 & -0.1138 & 0.7204 & -0.2014 & -0.4565 & 0.8952 & -0.3630 & 0.1860 & 0.0917 \\
\hline & $(-2.65)$ & $(-0.31)$ & $(2.80)$ & $(-1.43)$ & $(-1.94)$ & $(2.41)$ & $(-2.64)$ & $(1.20)$ & (1.05) \\
\hline $\operatorname{Adj} R^{2}(3)$ & 0.9519 & 0.8729 & 0.9143 & 0.9676 & 0.9419 & 0.9124 & 0.9663 & 0.9684 & 0.9793 \\
\hline
\end{tabular}




\begin{tabular}{|c|c|c|c|c|c|c|c|c|c|}
\hline \multicolumn{10}{|c|}{ Panel C: Four-factor CAPM performance } \\
\hline \multirow[t]{2}{*}{$\hat{\alpha}$} & -0.0022 & 0.0036 & 0.0035 & -0.0010 & -0.0020 & 0.0030 & -0.0033 & -0.0002 & -0.0023 \\
\hline & $(-2.34)$ & $(1.75)$ & $(2.49)$ & $(-1.30)$ & $(-1.64)$ & $(1.86)$ & $(-3.96)$ & $(-0.19)$ & $(-3.43)$ \\
\hline \multirow[t]{2}{*}{$\hat{\beta}$} & 1.0079 & 1.0099 & 0.9680 & 0.9908 & 1.0139 & 0.9941 & 0.9661 & 1.0367 & 0.9929 \\
\hline & $(46.92)$ & $(31.54)$ & $(42.69)$ & $(63.18)$ & $(43.16)$ & $(37.66)$ & $(59.71)$ & $(62.02)$ & $(78.84)$ \\
\hline \multirow[t]{2}{*}{$\hat{s}$} & 0.5632 & 0.7016 & 0.4201 & -0.1295 & 0.1680 & -0.2289 & -0.6661 & -0.2469 & -0.4128 \\
\hline & $(2.01)$ & (1.41) & $(1.72)$ & $(-1.00)$ & $(0.63)$ & $(-1.08)$ & $(-3.74)$ & $(-1.83)$ & $(-5.71)$ \\
\hline \multirow{2}{*}{$\hat{h}$} & -0.7393 & -0.0784 & 0.7139 & -0.1667 & -0.4703 & 0.8743 & -0.3760 & 0.1972 & 0.1022 \\
\hline & $(-2.83)$ & $(-0.22)$ & $(2.85)$ & $(-1.35)$ & $(-1.96)$ & $(2.43)$ & $(-2.63)$ & $(1.31)$ & $(1.26)$ \\
\hline \multirow[t]{2}{*}{$\hat{z}$} & -0.0982 & 0.1183 & -0.0218 & 0.1162 & -0.0662 & -0.0699 & -0.0435 & 0.0373 & 0.0353 \\
\hline & $(-2.09)$ & (1.48) & $(-0.39)$ & $(3.05)$ & $(-1.09)$ & $(-1.31)$ & $(-0.76)$ & $(0.85)$ & (1.03) \\
\hline $\operatorname{Adj} R^{2}(4)$ & 0.9533 & 0.8743 & 0.9136 & 0.9702 & 0.9422 & 0.9125 & 0.9664 & 0.9684 & 0.9794 \\
\hline
\end{tabular}


Table 9 Pooled cross-section regression for equally weighted monthly excess returns on country portfolios with size and illiquidity for 1996 to 2007

This table contrasts the performance of the one factor CAPM model with its multifactor counterparts augmented by each of the additional three factors, size, liquidity and legal regime in turn. Regression results are presented for aggregate market portfolios for each of the six sample group markets. The four factor CAPM:

$r_{i t}-r_{f t}=\alpha_{i}+\beta_{i}\left(r_{m t}-r_{f t}\right)+s_{i} S M B_{t}+h_{i} H M L_{t}+z_{i} L E G A L_{t}+\varepsilon_{i t}$

where $r_{i t}$ is the return of portfolio i in month $t, r_{f t}$ is the one month T-bill risk free rate for month $t$, which is taken as the one month UK Gilt rate in this case. Numbers in parentheses are Newey-West HAC covariance adjusted t-statistics.

\section{Explanatory Variables \\ Panel 1: Austria (ATX 50 constituents)}

CAPM

CAPM and SMB and ILLIQ

CAPM and SMB and ILLIQ and LEGAL

Panel 2: Belgium (BEL 20 constituents)

CAPM

CAPM and SMB and ILLIQ

CAPM and SMB and ILLIQ and LEGAL

Panel 3: Denmark (OMX Copenhagen constituents)

CAPM

CAPM and SMB and ILLIQ

CAPM and SMB and ILLIQ and LEGAL

Panel 4: Finland (OMX Helsinki constituents)

CAPM

CAPM and SMB and ILLIQ

CAPM and SMB and ILLIQ and LEGAL

Panel 5: France (CAC 40 constituents)

CAPM

CAPM and SMB and ILLIQ

CAPM and SMB and ILLIQ and LEGAL

Panel 6: Germany (DAX 100 constituents)

CAPM

CAPM and SMB and ILLIQ

CAPM and SMB and ILLIQ and LEGAL

Panel 7: Iceland (OMX Iceland constituents)

CAPM

CAPM and SMB and ILLIQ

CAPM and SMB and ILLIQ and LEGAL

Panel 8: Ireland (ISEQ Overall constituents)

CAPM

CAPM and SMB and ILLIQ

$\hat{\alpha}$

$-0.0046(-1.58)$

$-0.0046(-1.55)$

$-0.0054(-1.70)$

$-0.0096(-2.70)$

$-0.0111(-2.91)$

$-0.0109(-2.73)$

$0.0012(0.26)$

$0.0013(0.30)$

$0.0011(0.22)$

$-0.0034(-1.19)$

$-0.0032(-1.13)$

$-0.0037(-1.12)$

$-0.0114(-3.75)$

$-0.0129(-3.96)$

$-0.0129(-3.50)$

$-0.0086(-2.62)$

$-0.0095(-2.61)$

$-0.0093(-2.43)$

$-0.0034(-1.19)$

$-0.0032(-1.13)$

$-0.0037(-1.12)$

$-0.0056(-0.96)$

$-0.0041(-0.72)$

$-0.0037(-0.60)$

$\hat{\beta}$

$0.7137(7.60)$
$0.6990(7.25)$
$0.6905(7.23)$

$0.9388(9.24)$

$0.9343(9.10)$

0.9361 (9.41)

0.9595 (11.69)

0.9478 (11.19)

0.9450 (11.12)

0.9925 (19.73)

0.9866 (18.99)

0.9814 (17.92)

1.1970 (13.48)

1.1916 (14.55)

1.1919 (14.84)

1.1446 (11.69)

1.1331 (12.14)

1.1351 (12.48)

$0.9925(19.73)$

0.9866 (18.99)

0.9814 (17.92)

$1.0913(7.26)$

$1.0863(7.33)$

$1.0918(7.16)$

$\hat{S}$

(n)

$-0.5154(-2.00)$

-0.5380 (-2.01)

$-0.0755(-0.24)$

$-0.0707(-0.23)$

$-0.4241(-2.05)$

$-0.4316(-2.04)$

$-0.2201(-1.20)$

$-0.2340(-1.24)$

$-0.1029(-0.36)$

$-0.1020(-0.35)$

$-0.3540(-1.26)$

$-0.3488(-1.27)$

$-0.2201(-1.20)$

$-0.2340(-1.24)$

$-0.2595(-0.93)$

$-0.2450(-0.91)$
$0.0761(0.55)$

$0.0440(0.27)$

$-0.4528(-1.88)$

$-0.4460(-1.74)$

$0.1328(1.02)$

$0.1222(0.88)$

$0.0963(0.98)$

$0.0766(0.66)$

$-0.4769(-2.01)$

-0.4757 (-1.89)

$-0.2231(-1.26)$

$-0.2157(-1.14)$

$0.0963(0.98)$

$0.0766(0.66)$

0.5094 (3.61)

0.5301 (3.50) $\hat{z}$

Adj R ${ }^{2}$

0.5580

0.5665

0.5652

0.6016

0.6045

0.6009

0.6128

0.6132

0.6098

0.7146

0.7118

0.7098

0.7334

0.7372

0.7347

0.7331

0.7341

0.7318

0.7146

0.7146
0.7118

0.7098

0.5818

0.5850

0.5817 


\begin{tabular}{|c|c|c|c|c|c|c|}
\hline Explanatory Variables & $\hat{\alpha}$ & $\hat{\beta}$ & $\hat{s}$ & $\hat{h}$ & $\hat{z}$ & $\operatorname{Adj} R^{2}$ \\
\hline \multicolumn{7}{|l|}{ Panel 9: Italy (FTSE STAR constituents) } \\
\hline CAPM & $-0.0127(-3.23)$ & $1.1470(20.67)$ & & & & 0.6738 \\
\hline CAPM and SMB and ILLIQ & $-0.0128(-3.18)$ & $1.1355(20.53)$ & $-0.3976(-1.57)$ & $0.0252(0.12)$ & & 0.6724 \\
\hline CAPM and SMB and ILLIQ and LEGAL & $-0.0146(-3.29)$ & $1.1152(20.00)$ & $-0.4513(-1.80)$ & $-0.0511(-0.26)$ & $-0.2556(-1.61)$ & 0.6764 \\
\hline \multicolumn{7}{|c|}{ Panel 10: Luxembourg (Luxembourg constituents) } \\
\hline CAPM & $-0.0124(-2.72)$ & $1.3006(11.27)$ & & & & 0.6904 \\
\hline CAPM and SMB and ILLIQ & $-0.0132(-2.88)$ & $1.2832(11.32)$ & $-0.5647(-1.79)$ & $-0.1827(-1.03)$ & & 0.6930 \\
\hline CAPM and SMB and ILLIQ and LEGAL & $-0.0145(-2.98)$ & $1.2684(11.80)$ & $-0.6036(-1.86)$ & $-0.2380(-1.16)$ & $-0.1852(-1.43)$ & 0.6931 \\
\hline \multicolumn{7}{|c|}{ Panel 11: Netherlands (Amsterdam All Share constituents) } \\
\hline CAPM & $-0.0117(-3.92)$ & 1.1558 (19.52) & & & & 0.7683 \\
\hline CAPM and SMB and ILLIQ & $-0.0124(-3.97)$ & $1.1464(19.89)$ & $-0.2912(-1.06)$ & $-0.1811(-1.13)$ & & 0.7681 \\
\hline CAPM and SMB and ILLIQ and LEGAL & $-0.0125(-3.95)$ & $1.1446(19.99)$ & $-0.2960(-1.05)$ & $-0.1878(-1.07)$ & $-0.0226(-0.19)$ & 0.7660 \\
\hline \multicolumn{7}{|l|}{ Panel 12: Norway (Oslo constituents) } \\
\hline CAPM & $-0.0059(-1.14)$ & $1.5291(14.69)$ & & & & 0.6938 \\
\hline CAPM and SMB and ILLIQ & $-0.0058(-1.15)$ & $1.5086(13.56)$ & $-0.7311(-2.48)$ & $0.1617(0.80)$ & & 0.6975 \\
\hline CAPM and SMB and ILLIQ and LEGAL & $-0.0051(-0.93)$ & $1.5168(13.59)$ & $-0.7094(-2.34)$ & $0.1926(0.94)$ & $0.1034(0.53)$ & 0.6954 \\
\hline \multicolumn{7}{|c|}{ Panel 13: Portugal (Portugal PSI General constituents) } \\
\hline CAPM & $-0.0067(-1.65)$ & $0.8860(13.54)$ & & & & 0.5500 \\
\hline CAPM and SMB and ILLIQ & $-0.0072(-1.74)$ & $0.8636(13.76)$ & $-0.7555(-2.60)$ & $-0.0550(-0.28)$ & & 0.5642 \\
\hline CAPM and SMB and ILLIQ and LEGAL & $-0.0093(-2.36)$ & $0.8397(13.95)$ & $-0.8188(-2.69)$ & $-0.1449(-0.75)$ & $-0.3012(-2.19)$ & 0.5736 \\
\hline \multicolumn{7}{|c|}{ Panel 14: Slovenia (SBI20 Index constituents) } \\
\hline CAPM & $0.0018(0.21)$ & $0.5736(3.81)$ & & & & 0.2072 \\
\hline CAPM and SMB and ILLIQ & $0.0016(0.20)$ & $0.5592(3.62)$ & $-0.4991(-1.43)$ & $0.0258(0.06)$ & & 0.2017 \\
\hline CAPM and SMB and ILLIQ and LEGAL & $0.0011(0.13)$ & $0.5531(3.76)$ & $-0.5152(-1.43)$ & $0.0029(0.01)$ & $-0.0767(-0.35)$ & 0.1951 \\
\hline \multicolumn{7}{|l|}{ Panel 15: Spain (IGBM Index constituents) } \\
\hline CAPM & $-0.0028(-1.12)$ & $0.7649(13.66)$ & & & & 0.6165 \\
\hline CAPM and SMB and ILLIQ & $-0.0031(-1.19)$ & $0.7510(13.68)$ & $-0.4687(-2.25)$ & $-0.0319(-0.19)$ & & 0.6225 \\
\hline CAPM and SMB and ILLIQ and LEGAL & $-0.0040(-1.50)$ & $0.7402(14.07)$ & $-0.4972(-2.32)$ & $-0.0725(-0.42)$ & $-0.1360(-1.24)$ & 0.6231 \\
\hline \multicolumn{7}{|c|}{ Panel 16: Sweden (OMX Stockholm constituents) } \\
\hline CAPM & $-0.0083(-2.77)$ & $1.3660(18.85)$ & & & & 0.7762 \\
\hline CAPM and SMB and ILLIQ & $-0.0092(-3.06)$ & $1.3530(18.76)$ & $-0.4316(-1.67)$ & $-0.2306(-0.92)$ & & 0.7780 \\
\hline CAPM and SMB and ILLIQ and LEGAL & $-0.0107(-3.29)$ & $1.3351(19.19)$ & $-0.4788(-1.70)$ & $-0.2977(-1.14)$ & $-0.2245(-1.54)$ & 0.7804 \\
\hline \multicolumn{7}{|c|}{ Panel 17: Switzerland (Swiss Leadership Index constituents) } \\
\hline CAPM & $-0.0080(-2.07)$ & $1.0762(11.34)$ & & & & 0.6958 \\
\hline CAPM and SMB and ILLIQ & $-0.0095(-2.44)$ & $1.0734(11.46)$ & $-0.0141(-0.06)$ & $-0.4824(-2.70)$ & & 0.7003 \\
\hline CAPM and SMB and ILLIQ and LEGAL & $-0.0092(-2.12)$ & $1.0770(12.11)$ & $-0.0046(-0.02)$ & $-0.4689(-2.38)$ & $0.0450(0.27)$ & 0.6977 \\
\hline \multicolumn{7}{|l|}{ Panel 18: UK (FTSE100 constituents) } \\
\hline CAPM & $-0.0054(-2.15)$ & $0.8626(16.71)$ & & & & 0.7587 \\
\hline CAPM and SMB and ILLIQ & $-0.0057(-2.13)$ & $0.8588(17.35)$ & $-0.1157(-0.50)$ & $-0.0655(-0.57)$ & & 0.7554 \\
\hline CAPM and SMB and ILLIQ and LEGAL & $-0.0049(-1.63)$ & $0.8683(17.93)$ & $-0.0906(-0.40)$ & $-0.0298(-0.22)$ & $0.1194(1.11)$ & 0.7562 \\
\hline
\end{tabular}




\begin{tabular}{|c|c|c|c|c|c|c|}
\hline Explanatory Variables & $\hat{\alpha}$ & $\hat{\beta}$ & $\hat{s}$ & $\hat{h}$ & $\hat{z}$ & $\operatorname{Adj} R^{2}$ \\
\hline \multicolumn{7}{|c|}{ Panel 19: Cyprus (CSE General constituents) } \\
\hline CAPM & $-0.0185(-1.99)$ & $1.0970(7.56)$ & & & & 0.2838 \\
\hline CAPM and SMB and ILLIQ & $-0.0168(-1.79)$ & $1.0880(7.74)$ & $-0.4149(-0.49)$ & $0.6002(1.37)$ & & 0.2791 \\
\hline CAPM and SMB and ILLIQ and LEGAL & $-0.0221(-2.37)$ & $1.0266(6.83)$ & $-0.5772(-0.68)$ & $0.3694(0.84)$ & $-0.7724(-2.73)$ & 0.3027 \\
\hline \multicolumn{7}{|c|}{ Panel 20: Greece (Athens Composite constituents) } \\
\hline CAPM & $-0.0157(-2.86)$ & $1.2889(9.11)$ & & & & 0.4446 \\
\hline CAPM and SMB and ILLIQ & $-0.0146(-2.79)$ & $1.2571(8.36)$ & $-1.1833(-1.96)$ & $0.5490(2.00)$ & & 0.4611 \\
\hline CAPM and SMB and ILLIQ and LEGAL & $-0.0160(-2.70)$ & $1.2419(7.62)$ & $-1.2235(-1.95)$ & $0.4918(1.69)$ & $-0.1914(-0.59)$ & 0.4582 \\
\hline \multicolumn{7}{|l|}{ Panel 21: Bulgaria (BG40 constituents) } \\
\hline CAPM & $0.0243(1.57)$ & $1.1089(4.36)$ & & & & 0.1467 \\
\hline CAPM and SMB and ILLIQ & $0.0224(1.38)$ & $1.0755(4.13)$ & $-1.0682(-1.23)$ & $-0.4257(-0.69)$ & & 0.1406 \\
\hline CAPM and SMB and ILLIQ and LEGAL & $0.0141(0.99)$ & $0.9791(4.38)$ & $-1.3232(-1.55)$ & $-0.7883(-1.03)$ & $-1.2139(-2.14)$ & 0.1715 \\
\hline \multicolumn{7}{|l|}{ Panel 22: Czech (Prague SE PX constituents) } \\
\hline CAPM & $0.0072(1.17)$ & $0.6765(4.94)$ & & & & 0.3155 \\
\hline CAPM and SMB and ILLIQ & $0.0068(1.07)$ & $0.6522(4.86)$ & $-0.8329(-1.98)$ & $0.0083(0.03)$ & & 0.3299 \\
\hline CAPM and SMB and ILLIQ and LEGAL & $0.0050(0.67)$ & $0.6312(4.99)$ & $-0.8883(-2.07)$ & $-0.0704(-0.25)$ & $-0.2637(-1.05)$ & 0.3340 \\
\hline \multicolumn{7}{|l|}{ Panel 23: Estonia (All share constituents) } \\
\hline CAPM & $0.0041(0.60)$ & $0.6301(3.71)$ & & & & 0.2448 \\
\hline CAPM and SMB and ILLIQ & $0.0036(0.51)$ & $0.6290(3.62)$ & $-0.0050(-0.01)$ & $-0.1906(-0.81)$ & & 0.2326 \\
\hline CAPM and SMB and ILLIQ and LEGAL & $0.0021(0.32)$ & $0.6126(3.63)$ & $-0.04836(-0.13)$ & $-0.2522(-0.97)$ & $-0.2060(-0.96)$ & 0.2312 \\
\hline \multicolumn{7}{|l|}{ Panel 24: Hungary (BUX constituents) } \\
\hline CAPM & $-0.0021(-0.31)$ & $1.0767(8.99)$ & & & & 0.3767 \\
\hline CAPM and SMB and ILLIQ & $-0.0004(-0.06)$ & $1.0728(8.88)$ & $-0.2368(-0.37)$ & $0.5880(1.15)$ & & 0.3744 \\
\hline CAPM and SMB and ILLIQ and LEGAL & $-0.0034(-0.43)$ & $1.0382(9.02)$ & $-0.3282(-0.48)$ & $0.4581(0.78)$ & $-0.4349(-1.48)$ & 0.3817 \\
\hline \multicolumn{7}{|c|}{ Panel 25: Poland (Warsaw General constituents) } \\
\hline CAPM & $-0.0016(-0.23)$ & $1.3942(11.49)$ & & & & 0.4342 \\
\hline CAPM and SMB and ILLIQ & $-0.0019(-0.26)$ & $1.3443(11.48)$ & $-1.7390(-3.80)$ & $0.1698(0.54)$ & & 0.4620 \\
\hline CAPM and SMB and ILLIQ and LEGAL & $-0.0034(-0.40)$ & $1.3278(10.13)$ & $-1.7827(-3.63)$ & $0.1076(0.31)$ & $-0.2080(-0.51)$ & 0.4591 \\
\hline \multicolumn{7}{|c|}{ Panel 26: Romania (CECE ROX constituents) } \\
\hline CAPM & $0.0101(0.71)$ & $1.1930(3.49)$ & & & & 0.2035 \\
\hline CAPM and SMB and ILLIQ & $0.0137(0.91)$ & $1.2022(3.56)$ & $0.1110(0.13)$ & $1.1714(1.02)$ & & 0.2035 \\
\hline CAPM and SMB and ILLIQ and LEGAL & $0.0095(0.61)$ & $1.1540(3.59)$ & $-0.0165(-0.01)$ & $0.9901(0.81)$ & $-0.6070(-1.49)$ & 0.2076 \\
\hline \multicolumn{7}{|c|}{ Panel 27: Russia (MICEX All Companies constituents) } \\
\hline CAPM & $0.0564(2.93)$ & $1.6254(3.92)$ & & & & 0.1135 \\
\hline CAPM and SMB and ILLIQ & $0.0809(3.99)$ & $1.7321(5.60)$ & $2.3200(1.30)$ & $7.6670(3.16)$ & & 0.3038 \\
\hline CAPM and SMB and ILLIQ and LEGAL & $0.0707(4.17)$ & $1.6135(6.03)$ & $2.0062(1.29)$ & $7.2208(3.41)$ & $-1.4938(-1.76)$ & 0.3189 \\
\hline \multicolumn{7}{|l|}{ Panel 28: Morocco (CFG 25 constituents) } \\
\hline CAPM & $0.0058(1.33)$ & $0.3653(3.821)$ & & & & 0.0979 \\
\hline CAPM and SMB and ILLIQ & $0.0056(1.27)$ & $0.3617(3.76)$ & $-0.1209(-0.36)$ & $-0.0209(-0.10)$ & & 0.0820 \\
\hline CAPM and SMB and ILLIQ and LEGAL & $0.0056(1.31)$ & $0.3617(3.70)$ & $-0.1210(-0.35)$ & $-0.0209(-0.10)$ & $-0.0001(-0.01)$ & 0.0735 \\
\hline
\end{tabular}




\begin{tabular}{|c|c|c|c|c|c|c|}
\hline Explanatory Variables & $\hat{\alpha}$ & $\hat{\beta}$ & $\hat{s}$ & $\hat{h}$ & $\hat{z}$ & $\operatorname{Adj} R^{2}$ \\
\hline \multicolumn{7}{|c|}{ Panel 29: Egypt (Hermes Financial constituents) } \\
\hline CAPM & $0.0109(1.02)$ & 1.1448 (6.69) & & & & 0.2714 \\
\hline CAPM and SMB and ILLIQ & $0.0131(1.14)$ & $1.1431(6.71)$ & $-0.1872(-0.26)$ & $0.7351(1.03)$ & & 0.2666 \\
\hline CAPM and SMB and ILLIQ and LEGAL & $0.0055(0.55)$ & $1.0549(6.74)$ & $-0.4205(-0.51)$ & $0.4034(0.52)$ & $-1.1104(-3.11)$ & 0.3148 \\
\hline \multicolumn{7}{|c|}{ Panel 30: South Africa (JSE Top 40 constituents) } \\
\hline CAPM & $-0.0010(-0.20)$ & $1.1298(14.35)$ & & & & 0.5502 \\
\hline CAPM and SMB and ILLIQ & $-0.0009(-0.18)$ & $1.1189(14.58)$ & $-0.3904(-1.10)$ & $0.0877(0.33)$ & & 0.5459 \\
\hline CAPM and SMB and ILLIQ and LEGAL & $0.0005(0.10)$ & $1.1360(14.33)$ & $-0.3450(-0.97)$ & $0.1523(0.59)$ & $0.2160(1.27)$ & 0.5459 \\
\hline \multicolumn{7}{|c|}{ Panel 31: Canada (S\&P/TSX Composite constituents) } \\
\hline CAPM & $0.0060(1.75)$ & $1.0354(15.06)$ & & & & 0.7102 \\
\hline CAPM and SMB and ILLIQ & $0.0052(1.47)$ & $1.0277(14.63)$ & $-0.2223(-1.19)$ & $-0.2379(-2.04)$ & & 0.7094 \\
\hline CAPM and SMB and ILLIQ and LEGAL & $0.0069(1.92)$ & $1.0478(14.29)$ & $-0.1692(-0.81)$ & $-0.1624(-1.22)$ & $0.2526(2.14)$ & 0.7156 \\
\hline \multicolumn{7}{|l|}{ Panel 32: US (S\&P 500 constituents) } \\
\hline CAPM & $-0.0019(-0.50)$ & $0.6390(7.74)$ & & & & 0.5021 \\
\hline CAPM and SMB and ILLIQ & $-0.0037(-1.08)$ & $0.6510(8.04)$ & $0.5288(4.37)$ & $-0.6851(-6.81)$ & & 0.5535 \\
\hline CAPM and SMB and ILLIQ and LEGAL & $-0.0016(-0.41)$ & $0.6755(8.89)$ & $0.5937(4.70)$ & $-0.5928(-4.20)$ & $0.3091(1.95)$ & 0.5743 \\
\hline \multicolumn{7}{|c|}{ Panel 33: Australia (S\&P/ASX 100 constituents) } \\
\hline CAPM & $-0.0003(-0.08)$ & $1.1077(11.71)$ & & & & 0.7148 \\
\hline CAPM and SMB and ILLIQ & $-0.0012(-0.33)$ & $1.0957(11.46)$ & $-0.3678(-1.74)$ & $-0.2506(-1.00)$ & & 0.7166 \\
\hline CAPM and SMB and ILLIQ and LEGAL & $-6.98 \mathrm{E}-05(-0.01)$ & $1.1096(11.63)$ & $-0.3310(-1.59)$ & $-0.1983(-0.86)$ & $0.1753(1.09)$ & 0.7178 \\
\hline \multicolumn{7}{|c|}{ Panel 34: New Zealand (NZX 50 Index constituents) } \\
\hline CAPM & $-0.0035(-0.82)$ & $0.7673(9.62)$ & & & & 0.4667 \\
\hline CAPM and SMB and ILLIQ & $-0.0048(-1.09)$ & $0.7633(9.48)$ & $-0.0608(-0.36)$ & $-0.4235(-1.74)$ & & 0.4674 \\
\hline CAPM and SMB and ILLIQ and LEGAL & $-0.0023(-0.54)$ & $0.7919(10.15)$ & $0.0146(0.07)$ & $-0.3162(-1.43)$ & $0.3593(2.14)$ & 0.4842 \\
\hline \multicolumn{7}{|l|}{ Panel 35: Argentina (All share constituents) } \\
\hline CAPM & $-0.0083(-1.05)$ & $0.4293(4.24)$ & & & & 0.0971 \\
\hline CAPM and SMB and ILLIQ & $-0.0098(-1.27)$ & $0.4446(4.71)$ & $0.6307(1.49)$ & $-0.6127(-1.90)$ & & 0.1083 \\
\hline CAPM and SMB and ILLIQ and LEGAL & $-0.0163(-1.67)$ & $0.3692(3.45)$ & $0.4311(1.10)$ & $-0.8965(-2.11)$ & $-0.9501(-1.88)$ & 0.2084 \\
\hline \multicolumn{7}{|l|}{ Panel 36: Brazil (IBX Index constituents) } \\
\hline CAPM & $0.0036(0.53)$ & $1.7175(9.05)$ & & & & 0.4958 \\
\hline CAPM and SMB and ILLIQ & $0.0078(0.93)$ & $1.7262(8.75)$ & $0.0603(0.05)$ & $1.3595(1.31)$ & & 0.5089 \\
\hline CAPM and SMB and ILLIQ and LEGAL & $0.0042(0.54)$ & $1.6849(8.74)$ & $-0.0487(-0.05)$ & $1.2043(1.24)$ & $-0.5194(-1.61)$ & 0.5140 \\
\hline \multicolumn{7}{|l|}{ Panel 37: Chile (IGPA Index constituents) } \\
\hline CAPM & $0.0002(0.07)$ & $0.7818(8.93)$ & & & & 0.5229 \\
\hline CAPM and SMB and ILLIQ & $-0.0009(-0.26)$ & $0.7767(9.17)$ & $-0.1057(-0.43)$ & $-0.3947(-2.06)$ & & 0.5243 \\
\hline CAPM and SMB and ILLIQ and LEGAL & $-0.0015(-0.38)$ & $0.7707(9.43)$ & $-0.1215(-0.49)$ & $-0.4172(-2.07)$ & $-0.0751(-0.45)$ & 0.5209 \\
\hline \multicolumn{7}{|l|}{ Panel 38: Colombia (All share constituents) } \\
\hline CAPM & $0.0073(1.12)$ & $0.7812(7.66)$ & & & & 0.3148 \\
\hline CAPM and SMB and ILLIQ & $0.0074(1.06)$ & $0.7765(7.67)$ & $-0.1728(-0.53)$ & $0.0871(0.26)$ & & 0.3034 \\
\hline CAPM and SMB and ILLIQ and LEGAL & $0.0053(0.83)$ & $0.7511(7.72)$ & $-0.2400(-0.64)$ & $-0.0083(-0.02)$ & $-0.3199(-1.68)$ & 0.3082 \\
\hline
\end{tabular}




\begin{tabular}{|c|c|c|c|c|c|c|}
\hline Explanatory Variables & $\hat{\alpha}$ & $\hat{\beta}$ & $\hat{s}$ & $\hat{h}$ & $\hat{z}$ & $\overline{\operatorname{Adj} R^{2}}$ \\
\hline \multicolumn{7}{|l|}{ Panel 39: Jamaica (All share constituents) } \\
\hline CAPM & $0.0343(1.88)$ & $0.2862(1.93)$ & & & & 0.0060 \\
\hline CAPM and SMB and ILLIQ & $0.0332(1.82)$ & $0.3340(2.28)$ & $1.7444(1.60)$ & $-0.6223(-0.98)$ & & 0.0199 \\
\hline CAPM and SMB and ILLIQ and LEGAL & $0.0404(1.60)$ & $0.4174(2.01)$ & $1.9650(1.52)$ & $-0.3085(-0.55)$ & $1.0503(0.78)$ & 0.0363 \\
\hline \multicolumn{7}{|c|}{ Panel 40: Mexico (IPC CompMx constituents) } \\
\hline CAPM & $-0.0049(-0.98)$ & $1.2778(15.84)$ & & & & 0.6496 \\
\hline CAPM and SMB and ILLIQ & $-0.0052(-1.05)$ & $1.2648(15.62)$ & $-0.4437(-1.34)$ & $-0.0191(-0.06)$ & & 0.6475 \\
\hline CAPM and SMB and ILLIQ and LEGAL & $-0.0044(-0.78)$ & $1.2745(15.47)$ & $-0.4181(-1.35)$ & $0.0172(0.06)$ & $0.1217(0.55)$ & 0.6455 \\
\hline \multicolumn{7}{|l|}{ Panel 41: Peru (All Share constituents) } \\
\hline CAPM & $0.0196(3.92)$ & $0.5601(7.80)$ & & & & 0.2323 \\
\hline CAPM and SMB and ILLIQ & $0.0197(3.57)$ & $0.5634(7.32)$ & $0.1103(0.25)$ & $0.0259(0.07)$ & & 0.2188 \\
\hline CAPM and SMB and ILLIQ and LEGAL & $0.0161(3.22)$ & $0.5216(7.12)$ & $-0.0003(-0.01)$ & $-0.1314(-0.29)$ & $-0.5271(-2.33)$ & 0.2561 \\
\hline \multicolumn{7}{|l|}{ Panel 42: Venezuela (All Share constituents) } \\
\hline CAPM & $0.0156(1.61)$ & $0.6047(2.50)$ & & & & 0.0539 \\
\hline CAPM and SMB and ILLIQ & $0.0064(0.72)$ & $0.5292(2.48)$ & $-2.1242(-1.02)$ & $-2.6559(-1.50)$ & & 0.1652 \\
\hline CAPM and SMB and ILLIQ and LEGAL & $-0.0010(-0.11)$ & $0.4421(2.21)$ & $-2.3545(-1.15)$ & $-2.9835(-1.73)$ & $-1.0963(-1.73)$ & 0.2006 \\
\hline \multicolumn{7}{|l|}{ Panel 43: Japan (TOPIX 100 constituents) } \\
\hline CAPM & $-0.0093(-2.33)$ & $0.7768(10.97)$ & & & & 0.4162 \\
\hline CAPM and SMB and ILLIQ & $-0.0085(-2.08)$ & $0.7914(11.06)$ & $0.4699(1.31)$ & $0.1767(0.75)$ & & 0.4156 \\
\hline CAPM and SMB and ILLIQ and LEGAL & $-0.0074(-1.57)$ & $0.8040(11.00)$ & $0.5031(1.41)$ & $0.2239(1.01)$ & $0.1578(0.61)$ & 0.4138 \\
\hline \multicolumn{7}{|c|}{ Panel 44: Singapore (SES Main board constituents) } \\
\hline CAPM & $-0.0051(-1.95)$ & $0.8734(16.63)$ & & & & 0.7984 \\
\hline CAPM and SMB and ILLIQ & $-0.0060(-2.35)$ & $0.8754(16.70)$ & $0.1251(0.75)$ & $-0.3168(-2.48)$ & & 0.8031 \\
\hline CAPM and SMB and ILLIQ and LEGAL & $-0.0016(-0.66)$ & $0.9272(23.79)$ & $0.2619(2.41)$ & $-0.1221(-1.51)$ & $0.6515(9.08)$ & 0.8949 \\
\hline \multicolumn{7}{|c|}{ Panel 45: Bangladesh (All share constituents) } \\
\hline CAPM & $0.0167(2.14)$ & $-0.0452(-0.42)$ & & & & 0.0011 \\
\hline CAPM and SMB and ILLIQ & $0.0154(1.97)$ & $-0.0382(-0.37)$ & $0.3254(0.87)$ & $-0.4903(-1.68)$ & & 0.0135 \\
\hline CAPM and SMB and ILLIQ and LEGAL & $0.0179(2.03)$ & $-0.0087(-0.08)$ & $0.4035(0.91)$ & $-0.3793(-1.13)$ & $0.3715(1.30)$ & 0.0291 \\
\hline \multicolumn{7}{|c|}{ Panel 46: China Shenzen (Shenzen 100 constituents) } \\
\hline CAPM & $0.0112(1.01)$ & $0.6205(3.83)$ & & & & 0.0987 \\
\hline CAPM and SMB and ILLIQ & $0.0128(1.19)$ & $0.5904(3.55)$ & $-1.1558(-1.53)$ & $0.7172(1.52)$ & & 0.1128 \\
\hline CAPM and SMB and ILLIQ and LEGAL & $0.0049(0.50)$ & $0.4992(3.31)$ & $-1.3971(-1.53)$ & $0.3741(0.59)$ & $-1.1482(-3.21)$ & 0.1815 \\
\hline \multicolumn{7}{|c|}{ Panel 47: China Shanghai (Shanghai 180 constituents) } \\
\hline CAPM & $0.0092(0.86)$ & $0.6266(3.88)$ & & & & 0.1032 \\
\hline CAPM and SMB and ILLIQ & $0.0106(1.02)$ & $0.5912(3.61)$ & $-1.3314(-1.86)$ & $0.6750(1.50)$ & & 0.1236 \\
\hline CAPM and SMB and ILLIQ and LEGAL & $0.0024(0.25)$ & $0.4954(3.43)$ & $-1.5848(-1.76)$ & $0.3148(0.50)$ & $-1.2059(-3.04)$ & 0.2022 \\
\hline \multicolumn{7}{|c|}{ Panel 48: Hong Kong (HS Composite constituents) } \\
\hline CAPM & $0.0011(0.28)$ & $1.3404(15.21)$ & & & & 0.6898 \\
\hline CAPM and SMB and ILLIQ & $0.0012(0.32)$ & $1.3072(14.66)$ & $-1.1749(-3.75)$ & $0.2242(0.89)$ & & 0.7149 \\
\hline CAPM and SMB and ILLIQ and LEGAL & $0.0061(1.61)$ & $1.3634(14.79)$ & $-1.0263(-3.92)$ & $0.4356(2.41)$ & $0.7075(4.63)$ & 0.7528 \\
\hline
\end{tabular}




\begin{tabular}{|c|c|c|c|c|c|c|}
\hline Explanatory Variables & $\hat{\alpha}$ & $\hat{\beta}$ & $\hat{s}$ & $\hat{h}$ & $\hat{z}$ & $\operatorname{Adj} R^{2}$ \\
\hline \multicolumn{7}{|l|}{ Panel 49: India (Bombay 100 constituents) } \\
\hline CAPM & $0.0093(1.25)$ & $1.6213(11.79)$ & & & & 0.5502 \\
\hline CAPM and SMB and ILLIQ & $0.0080(1.02)$ & $1.6031(11.23)$ & $-0.5672(-1.42)$ & $-0.3332(-0.99)$ & & 0.5474 \\
\hline CAPM and SMB and ILLIQ and LEGAL & $0.0118(1.40)$ & $1.6477(10.66)$ & $-0.4492(-1.19)$ & $-0.1654(-0.47)$ & $0.5618(1.49)$ & 0.5572 \\
\hline \multicolumn{7}{|c|}{ Panel 50: Indonesia (Jakarta Kompas 100 constituents) } \\
\hline CAPM & $-0.0267(-3.38)$ & $1.2340(5.73)$ & & & & 0.3656 \\
\hline CAPM and SMB and ILLIQ & $-0.0268(-3.49)$ & $1.2185(5.73)$ & $-0.5375(-1.13)$ & $0.0363(0.12)$ & & 0.3579 \\
\hline CAPM and SMB and ILLIQ and LEGAL & $-0.0278(-3.50)$ & $1.2069(5.89)$ & $-0.5680(-1.19)$ & $-0.0070(-0.02)$ & $-0.1451(-0.40)$ & 0.3530 \\
\hline \multicolumn{7}{|l|}{ Panel 51: Malaysia (Composite constituents) } \\
\hline CAPM & $-0.0053(-1.13)$ & $0.7831(9.39)$ & & & & 0.3895 \\
\hline CAPM and SMB and ILLIQ & $-0.0056(-1.19)$ & $0.7766(8.78)$ & $-0.2151(-0.55)$ & $-0.0378(-0.15)$ & & 0.3800 \\
\hline CAPM and SMB and ILLIQ and LEGAL & $-0.0025(-0.54)$ & $0.8125(9.62)$ & $-0.1201(-0.32)$ & $0.0973(0.42)$ & $0.4524(2.29)$ & 0.4020 \\
\hline \multicolumn{7}{|c|}{ Panel 52: Pakistan (Karachi 100 constituents) } \\
\hline CAPM & $0.0178(2.23)$ & $0.4245(2.00)$ & & & & 0.0533 \\
\hline CAPM and SMB and ILLIQ & $0.0187(2.44)$ & $0.4269(1.97)$ & $0.0279(0.04)$ & $0.3082(0.54)$ & & 0.0383 \\
\hline CAPM and SMB and ILLIQ and LEGAL & $0.0212(2.38)$ & $0.4562(2.04)$ & $0.1053(0.17)$ & $0.4182(0.71)$ & $0.3683(0.74)$ & 0.0392 \\
\hline \multicolumn{7}{|c|}{ Panel 53: Philippines (Manila All share constituents) } \\
\hline CAPM & $0.0138(1.58)$ & $1.0366(6.55)$ & & & & 0.1937 \\
\hline CAPM and SMB and ILLIQ & $0.0084(1.15)$ & $1.15(7.20)$ & $4.4043(2.45)$ & $-2.4978(-1.62)$ & & 0.4620 \\
\hline CAPM and SMB and ILLIQ and LEGAL & $0.0026(0.35)$ & $1.0853(7.47)$ & $4.2265(2.56)$ & $-2.7506(-1.78)$ & $-0.8461(-1.81)$ & 0.4852 \\
\hline \multicolumn{7}{|c|}{ Panel 54: South Korea (KOSPI 200 constituents) } \\
\hline CAPM & $-0.0014(-0.24)$ & $1.6980(11.06)$ & & & & 0.6332 \\
\hline CAPM and SMB and ILLIQ & $-0.0013(-0.20)$ & $1.6603(11.29)$ & $-1.3367(-2.82)$ & $0.2677(0.50)$ & & 0.6494 \\
\hline CAPM and SMB and ILLIQ and LEGAL & $-0.0016(-0.22)$ & $1.6564(10.99)$ & $-1.3470(-2.78)$ & $0.2530(0.44)$ & $-0.0491(-0.13)$ & 0.6463 \\
\hline \multicolumn{7}{|c|}{ Panel 55: Sri Lanka (Dow Jones Sri Lanka Titans 20 constituents) } \\
\hline CAPM & $0.0158(1.85)$ & $0.3085(2.08)$ & & & & 0.0146 \\
\hline CAPM and SMB and ILLIQ & $0.0156(1.74)$ & $0.3209(2.12)$ & $0.4444(0.67)$ & $-0.1292(-0.18)$ & & 0.0263 \\
\hline CAPM and SMB and ILLIQ and LEGAL & $0.0224(2.16)$ & $0.3998(2.62)$ & $0.6531(0.83)$ & $0.1675(0.26)$ & $0.9936(2.13)$ & 0.0415 \\
\hline \multicolumn{7}{|l|}{ Panel 56: Taiwan (Top 100 constituents) } \\
\hline CAPM & $-0.0053(-0.89)$ & $1.0831(9.86)$ & & & & 0.3671 \\
\hline CAPM and SMB and ILLIQ & $-0.0052(-0.8)$ & $1.0703(9.81)$ & $-0.4571(-1.24)$ & $0.1071(0.43)$ & & 0.3594 \\
\hline CAPM and SMB and ILLIQ and LEGAL & $-0.0008(-0.12)$ & $1.1216(11.37)$ & $-0.3213(-0.82)$ & $0.3001(1.25)$ & $0.6461(2.19)$ & 0.3814 \\
\hline \multicolumn{7}{|l|}{ Panel 57: Thailand (SET 100 constituents) } \\
\hline CAPM & $1.94 \mathrm{E}-05(0.01)$ & $1.3444(11.30)$ & & & & 0.4473 \\
\hline CAPM and SMB and ILLIQ & $0.0009(0.11)$ & $1.3519(11.12)$ & $0.2065(0.36)$ & $0.2792(0.66)$ & & 0.4391 \\
\hline CAPM and SMB and ILLIQ and LEGAL & $0.0078(0.98)$ & $1.4317(11.95)$ & $0.4177(0.81)$ & $0.5795(1.74)$ & $1.0054(3.59)$ & 0.4871 \\
\hline \multicolumn{7}{|c|}{ Panel 59: Israel (Dow Jones TA 100 constituents) } \\
\hline CAPM & $1.81 \mathrm{E}-05(0.01)$ & $0.9032(7.60)$ & & & & 0.3191 \\
\hline CAPM and SMB and ILLIQ & $-0.0009(-0.17)$ & $0.8837(7.35)$ & $-0.6329(-1.00)$ & $-0.2036(-0.65)$ & & 0.3167 \\
\hline CAPM and SMB and ILLIQ and LEGAL & $-0.0017(-0.30)$ & $0.8739(7.12)$ & $-0.6589(-1.01)$ & $-0.2407(-0.66)$ & $-0.1241(-0.46)$ & 0.3116 \\
\hline
\end{tabular}


CAPM and SMB and ILLIQ

$-0.0045(-0.31)$

$-0.0065(-0.50)$

$2.3948(5.97)$

CAPM and SMB and ILLIQ and LEGAL

$-0.0129(-0.94)$

$4.2714(1.30)$

$4.0737(1.25)$

$-1.3453(-0.67)$

$-1.6265(-0.74)$

0.2692

$*$ indicates models selected from which Cost of Equity are estimated 


\section{Table 10 Cost of Equity estimates}

This table presents the annualized cost of equity estimates generated at 05/2009 from the total risk premium, which is the sum of the constant, market premium and all relevant additional premiums (size, illiquidity and/or legal regime). The UK Gilt/ Treasury rate is used in each case for risk free rate. Values are percentages.

\begin{tabular}{|c|c|c|c|c|}
\hline Region & Market & $\begin{array}{l}\text { Cost of Equity } \\
(\%)\end{array}$ & Market & $\begin{array}{l}\text { Cost of Equity } \\
(\%)\end{array}$ \\
\hline \multirow[t]{9}{*}{ Europe Developed } & Austria & 13.87 & Luxembourg & 25.11 \\
\hline & Belgium & 18.22 & Netherlands & 21.30 \\
\hline & Denmark & 17.11 & Norway & 25.48 \\
\hline & Finland & 17.91 & Portugal & 18.83 \\
\hline & France & 22.72 & Slovenia & 11.50 \\
\hline & Germany & 20.93 & Spain & 15.30 \\
\hline & Iceland & 17.91 & Sweden & 26.61 \\
\hline & Ireland & 17.02 & Switzerland & 20.38 \\
\hline & Italy & 22.29 & UK & 14.84 \\
\hline \multirow[t]{5}{*}{ Europe Emerging } & Cyprus & 23.45 & Hungary & 20.42 \\
\hline & Greece & 22.69 & Poland & 26.24 \\
\hline & Bulgaria & 31.15 & Romania & 21.39 \\
\hline & Czech Rep. & 14.90 & Russia & 68.15 \\
\hline & Estonia & 13.93 & Slovakia & -- -- \\
\hline \multirow[t]{3}{*}{ Middle East and Africa } & Morocco & 7.42 & Israel & 18.20 \\
\hline & Egypt & 26.26 & Turkey & 50.49 \\
\hline & South Africa & 18.09 & & \\
\hline North America & Canada & 17.33 & United States & 11.53 \\
\hline Australasia & Australia & 19.25 & New Zealand & 12.65 \\
\hline \multirow[t]{4}{*}{ Latin America } & Argentina & 17.59 & Jamaica & 17.25 \\
\hline & Brazil & 28.70 & Mexico & 21.69 \\
\hline & Chile & 16.20 & Peru & 14.45 \\
\hline & Colombia & 16.41 & Venezuela & 30.53 \\
\hline Asia Developed & Japan & 14.62 & Singapore & 11.62 \\
\hline \multirow[t]{7}{*}{ Asia Emerging } & Bangladesh & -- -- & Malaysia & 10.88 \\
\hline & China Shenzen & 18.53 & Pakistan & -- -- \\
\hline & China Shanghai & 19.33 & Philippines & 31.42 \\
\hline & Hong Kong & 17.68 & South Korea & 29.42 \\
\hline & India & 25.11 & Sri Lanka & -- -- \\
\hline & Indonesia & 22.90 & Taiwan & 13.93 \\
\hline & & & Thailand & 14.44 \\
\hline
\end{tabular}


Figure 1. Market returns-based valuation factor

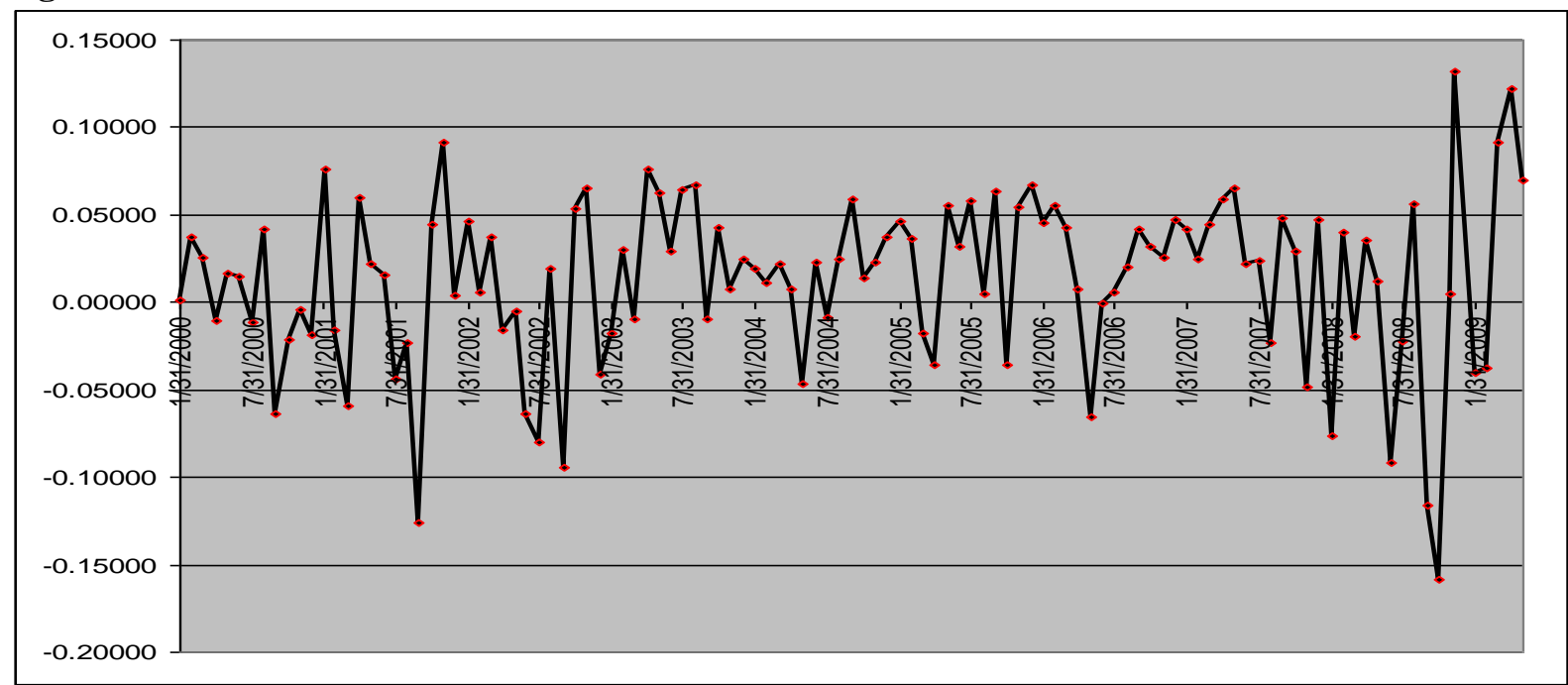

Figure 2. Size returns-based valuation factor

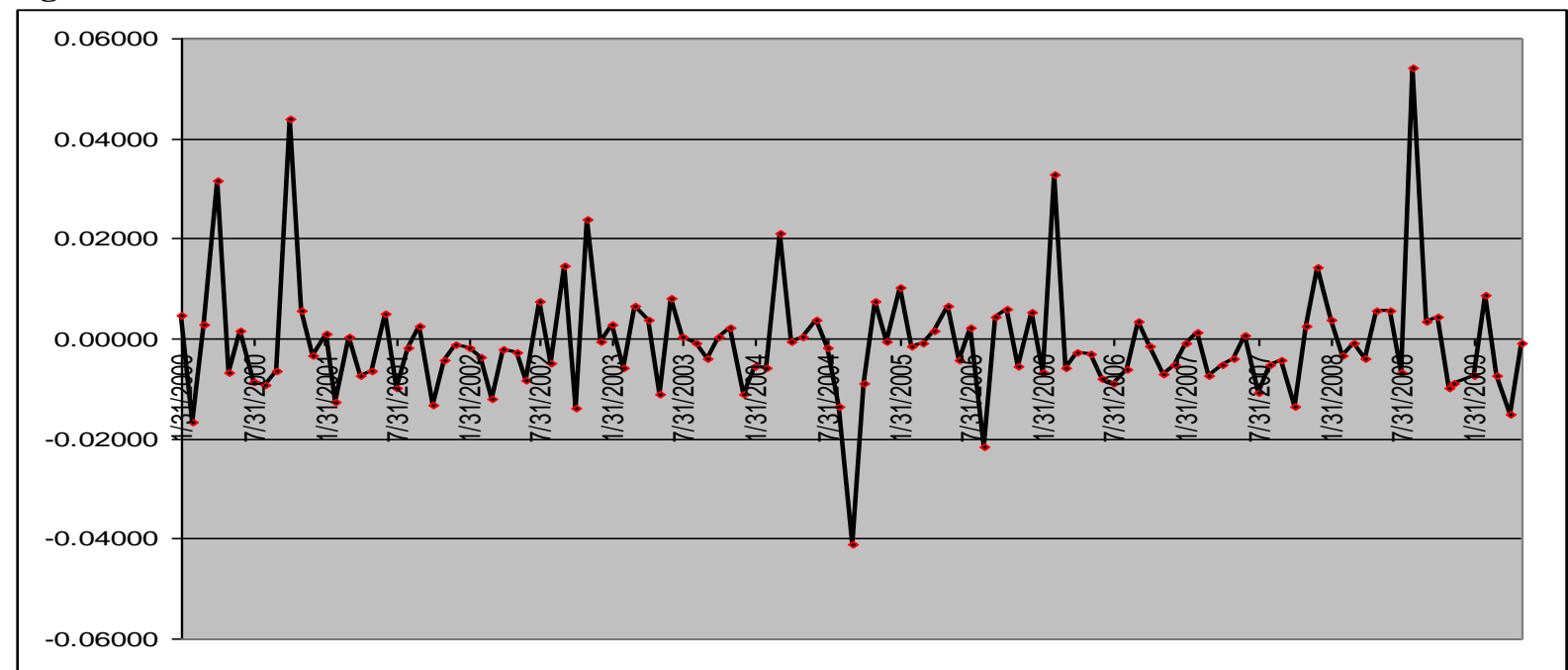

Figure 3. Illiquidity returns-based valuation factor

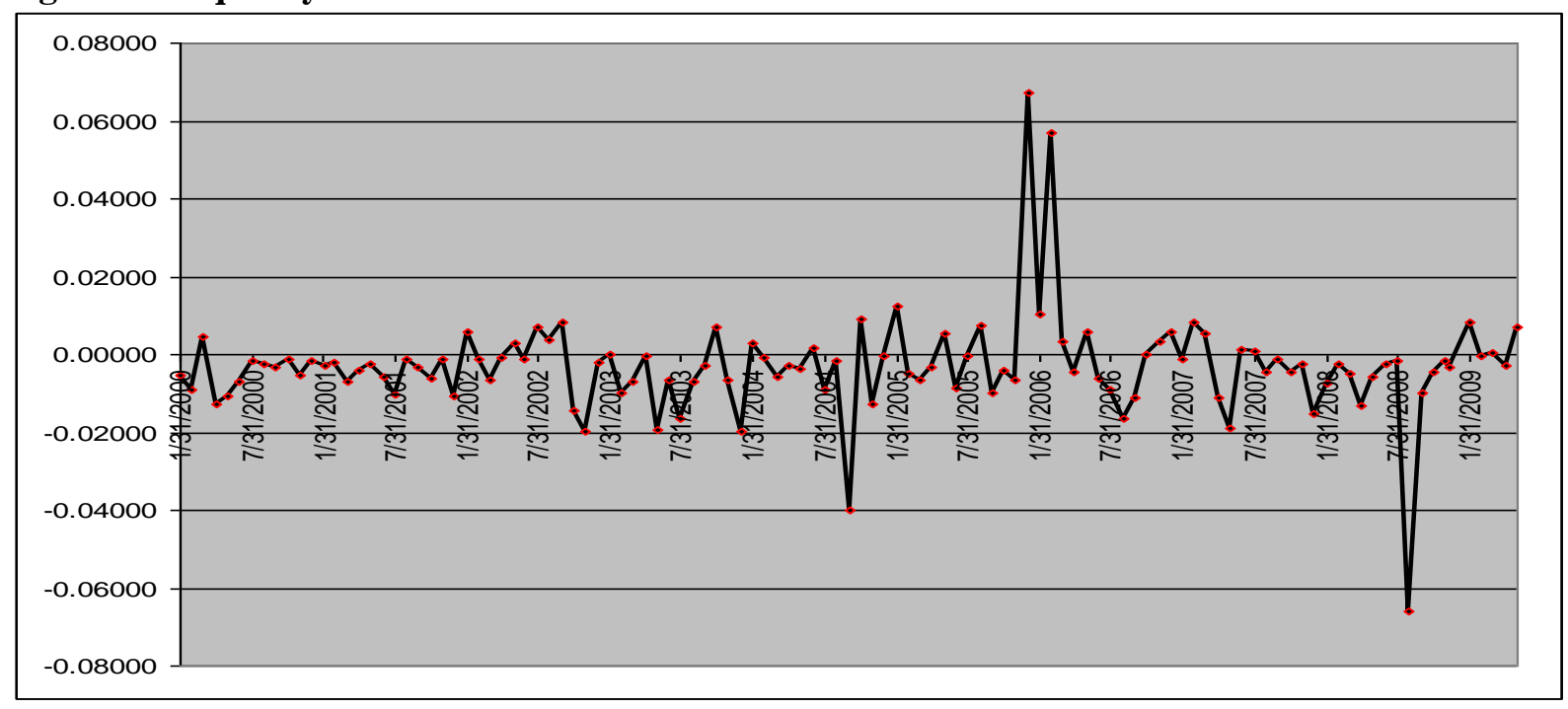


Figure 4. Legal Regime returns-based valuation factor

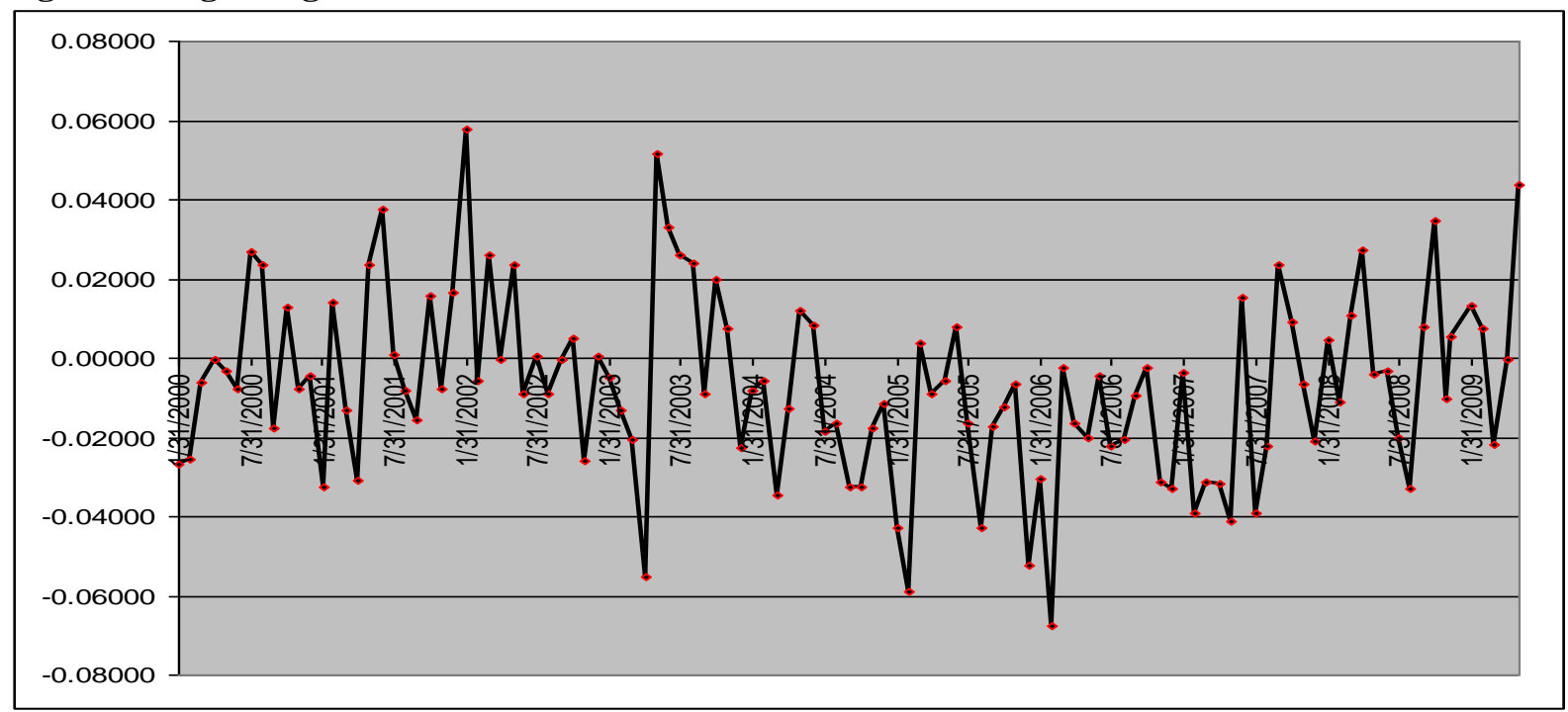




\begin{tabular}{|c|c|c|c|c|}
\hline Market & Legal Origin & Capital Gains Tax & Other Taxes and Fees & Commission \\
\hline Europe Developed & & & & \\
\hline Austria & German Civil Code & Exempt & None & $\begin{array}{l}\text { Transaction fee of } 0.00045 \% \text { as trading fee and } \\
\text { exchange transaction fee }\end{array}$ \\
\hline Belgium & French Civil Code & Exempt & $\begin{array}{l}\text { Dividends: } 15 \% \text { or } 25 \% \\
\text { Stamp duty: } 0.17 \%\end{array}$ & $\begin{array}{l}\text { Euronext Belgium Trades are fee free. However } \\
\text { order flow from agency is subject to } 0.00012 \% \text { levy } \\
\text { and from principal members a } 0.00006 \% \text { levy. }\end{array}$ \\
\hline Denmark & $\begin{array}{l}\text { Scandinavian Civil } \\
\text { Code }\end{array}$ & $\begin{array}{l}\text { Capital gains on sales of shares held by } \\
\text { individuals for less than } 3 \text { years are, for } \\
\text { residents, taxable as capital income, } \\
\text { with an income tax rate of between } 50 \\
\text { and } 56 \% \text {. If the shares have been held } \\
\text { for } 3 \text { years or more, a capital gain is } \\
\text { treated as dividend income, i.e. taxed at } \\
\text { a rate between } 30-40 \% \text {. }\end{array}$ & $\begin{array}{l}\text { Dividends on shares are in general } \\
\text { subject to a } 30 \% \text { withholding tax on } \\
\text { account. } \\
\text { Dividends received by residents are } \\
\text { taxed at rates between } 30 \text { and } 40 \%\end{array}$ & $\begin{array}{l}\text { OMX Nordic Total trading fee } 0.0000613 \% \\
\text { including trading fixed fee, electronic trading fee, } \\
\text { value based fees. }\end{array}$ \\
\hline Finland & $\begin{array}{l}\text { Scandinavian Civil } \\
\text { Code }\end{array}$ & Exempt & Dividends $29 \%$ & As OMX Nordic Denmark \\
\hline France & French Civil Code & $\begin{array}{l}\text { Capital gains above } 50,000 \text { are taxed at } \\
26 \%\end{array}$ & $\begin{array}{l}\text { There is a tax credit on dividends } \\
\text { ("avoir fiscal") }\end{array}$ & As Euronext Belgium \\
\hline Germany & German Civil Code & Exempt & None & $\begin{array}{l}\text { Xetra electronic trading fee of } 0.0048 \% \text { for trades } \\
\text { over EUR } 12,500 . \text { Broker fees subject to maximum } \\
\text { of } 0.8 \% \text { per trade }\end{array}$ \\
\hline Iceland & $\begin{array}{l}\text { Scandinavian Civil } \\
\text { Code }\end{array}$ & Exempt & None & As OMX Nordic Denmark \\
\hline Ireland & Common Law & $20 \%$ annual rate & $\begin{array}{l}\text { VAT at commission rate } 0.5 \% \\
\text { marketable security. } 1.0 \% \text { stamp duty } \\
\text { Investor Protection fee: } 0.0002 \%\end{array}$ & $\begin{array}{l}\text { Fixed membership annual fee of EUR } 25,000 \text { and } \\
\text { reporting fee of EUR } 1,000 \text {. Sliding scale of trade } \\
\text { fees per number shares traded from EUR } 1.50 \text { for } 0 \text { - } \\
1,000 \text { shares to EUR } 0.25 \text { for } 20,000+\text { shares. }\end{array}$ \\
\hline Italy & French Civil Code & Exempt & None & $\begin{array}{l}\text { Total trading costs approximately } 0.000061 \% \text { levied } \\
\text { against members initiating small trades of low value. } \\
\text { Figure includes clearing and settlement as well as } \\
\text { trading fees. }\end{array}$ \\
\hline Luxembourg & French Civil Code & Exempt & None & As Euronext Belgium \\
\hline Netherlands & French Civil Code & Exempt & None & As Euronext Belgium \\
\hline Norway & $\begin{array}{l}\text { Scandinavian Civil } \\
\text { Code }\end{array}$ & Exempt & $28 \%$ on dividends to private investors & $\begin{array}{l}\text { Value-based fee of } 0.0060 \% \text { and exchange reporting } \\
\text { fee of } 0.0024 \%\end{array}$ \\
\hline
\end{tabular}




\begin{tabular}{|c|c|c|c|c|}
\hline Market & Legal Origin & Capital Gains Tax & Other Taxes and Fees & Commission \\
\hline \multirow[t]{4}{*}{ Portugal } & French Civil Code & Shares held for more than 12 months & Witholding Tax Rates & As Euronext Belgium \\
\hline & & Residents $0 \%$ non-residents $25 \%$ & Received by investors & \\
\hline & & Shares held for less than 12 months & Dividends Residents $20 \%$ Non & \\
\hline & & Residents $10 \%$ non Residents $25 \%$ & Residents $20 \%$ & \\
\hline Slovenia & German Civil Code & Exempt & $20 \%$ Witholding tax & $\begin{array}{l}\text { LJSE transaction fee for shares is } 0.03 \% \text { of the value } \\
\text { of the transaction }\end{array}$ \\
\hline Spain & French Civil Code & Exempt & None & $\begin{array}{l}\text { Sliding scale of fixed trading fees with maximum } \\
\text { transaction fee of } 0.00004 \%\end{array}$ \\
\hline Sweden & $\begin{array}{l}\text { Scandinavian Civil } \\
\text { Code }\end{array}$ & Exempt & $\begin{array}{l}0-30 \% \text { witholding tax on dividends \& } \\
\text { interests paid on SEK denominated } \\
\text { securities to non-residents. } \\
\text { Tax reductions depend on bilateral tax } \\
\text { treaties. }\end{array}$ & As OMX Nordic Denmark \\
\hline Switzerland & German Civil Code & Exempt & None & $\begin{array}{l}\text { The maximum fee levied is that for foreign equities } \\
\text { in auction execution which is } 0.000065 \%\end{array}$ \\
\hline \multirow[t]{3}{*}{ UK } & Common Law & Exempt & None & $\begin{array}{l}\text { Total direct costs of trading (brokerage commission } \\
\text { and fees): } 0.0401 \%\end{array}$ \\
\hline & & & & Total indirect trading costs: $0.0101 \%$ \\
\hline & & & & Total trading costs: $0.0502 \%$ \\
\hline \multicolumn{5}{|r|}{ 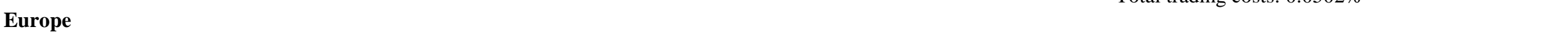 } \\
\hline \multicolumn{5}{|l|}{ Emerging } \\
\hline Cyprus & French Civil Code & Exempt & $\begin{array}{l}\text { Transaction Fees } 0.01 \% \text { on the total } \\
\text { transaction value. Central Depository } \\
\text { Fee for the clearing of transaction } \\
0.03 \% \text { on the total transaction value of } \\
\text { shares/rights/warrants } \\
\text { Government Sales tax }: 0.15 \% \text { on sales } \\
\text { transaction value }\end{array}$ & $\begin{array}{l}\text { Percentage variable charge } 0.04 \% \text { on the total } \\
\text { transaction value of shares/rights/warrants. For the } \\
\text { transactions in the CSE Main market the variable } \\
\text { charge is } 0.015 \% \text { on the total transaction value }\end{array}$ \\
\hline Greece & French Civil Code & Exempt & None & $\begin{array}{l}\text { Transaction fee of } 0.026 \% \text { and settlement costs of } \\
0.04 \%\end{array}$ \\
\hline Bulgaria & German Civil Code & Exempt & None & $\begin{array}{l}\text { Tiered transaction fees: On amount up to BGN } 25 \\
\text { million inclusive - } 0.1 \% \text {; } \\
\text { On amount above BGN } 25 \text { million up to BGN } 75 \\
\text { million inclusive - BGN } 25 \text { thousand plus } 0.05 \% \text { on } \\
\text { the excess over BGN } 25 \text { million; On amount above } \\
\text { BGN } 75 \text { million - BGN } 50 \text { thousand plus } 0.01 \% \text { on } \\
\text { the excess over } 75 \text { million }\end{array}$ \\
\hline Czech Rep. & German Civil Code & Exempt & None & $\begin{array}{l}\text { Transaction fee for organising trade including } \\
\text { settlement costs: } 0.07 \% \text { of trade value }\end{array}$ \\
\hline
\end{tabular}




\begin{tabular}{|c|c|c|c|c|}
\hline Market & Legal Origin & Capital Gains Tax & Other Taxes and Fees & Commission \\
\hline Estonia & German Civil Code & Exempt & None & $\begin{array}{l}\text { Nasdaq OMX Baltic Exchange fee of } 0.015 \% \text { of the } \\
\text { transaction value (i.e. transaction value in money } \\
\text { (EEK), which is equal to price of traded securities } \\
\text { multiplied by number of traded securities) but not } \\
\text { less than EEK } 5 \text { (Minimum fee) for each } \\
\text { automatically matched transaction }\end{array}$ \\
\hline Hungary & German Civil Code & $20 \%$ & $10 \%$ dividend tax & $\begin{array}{l}0.015 \% \text { of the transaction value expressed in forints, } \\
\text { with a minimum of HUF } 50 \text { and a maximum of HUF } \\
35,000\end{array}$ \\
\hline Poland & German Civil Code & $19 \%$ & Dividends: $19 \%$ & $\begin{array}{l}\text { Transaction fee of between } 0.033 \% \text { and } 0.010 \% \text { for } \\
\text { shares }\end{array}$ \\
\hline Romania & German Civil Code & Exempt & None & $\begin{array}{l}\text { Trading fee of } 0.3 \% \text { to seller and } 0.22 \% \text { to buyer } \\
\text { dropping to } 0.2 \% \text { and } 0.12 \% \text { for seller and buyer for } \\
\text { traded value under USD } 111,111.11 \text {. National } \\
\text { Securities commission also levies } 0.08 \% \text { trade fee. }\end{array}$ \\
\hline Russia & French Civil Code & Exempt & $\begin{array}{l}\text { Dividends: } 9 \% \text { - residents, } 15 \% \text { - non- } \\
\text { resident legal bodies, } 30 \% \text { - non- } \\
\text { resident individuals }\end{array}$ & N/A \\
\hline $\begin{array}{l}\text { Slovakia } \\
\text { Africa }\end{array}$ & German Civil Code & Exempt & None & Exchange Transaction fee of $0.08 \%$ \\
\hline Morocco & French Civil Code & Exempt & $\begin{array}{l}\text { VAT applied to the amount of } \\
\text { commissions is } 10 \% \text {. No other tax/ } \\
\text { fees. }\end{array}$ & $\begin{array}{l}\text { Standard fee of } 0.1 \% \text { of trade value in Moroccan } \\
\text { Dirhams (MAD) levied against buyers and sellers } \\
\text { engaging in securities transfer or dealing. This fee, } \\
\text { Negotiation des Titres, is applied to both buy and sell } \\
\text { legs of trade. }\end{array}$ \\
\hline Egypt & French Civil Code & Exempt & None & $\begin{array}{l}\text { Listed securities, the Exchange service fees are } \\
\text { levied at } 0.012 \% \text { of the value of each side of the } \\
\text { transaction with a maximum amount of LE (Egyptian } \\
\text { Pounds) } 5000 \text {. } \\
\text { Clearing fee: } 0.125 \text { per thousand of transaction value }\end{array}$ \\
\hline South Africa & Common Law & Exempt & $\begin{array}{l}\text { VAT at commission rate } 0.5 \% \\
\text { marketable security. } 1.0 \% \text { stamp duty } \\
\text { Investor Protection fee: } 0.0002 \%\end{array}$ & $\begin{array}{l}\text { Main Market: } 1.4 \% \text {, trades < R1,500,000 and } \\
0.21 \% \text {, trades > R1,500,000 } \\
\text { Equities main market minimum fee: R7.42 or } \\
\text { R8.46(incl. VAT) on both buy and sell legs of a } \\
\text { position } \\
\text { Clearing and Settlement Fee: } 0.0026 \% \text { Subject to } \\
\text { minimum of R2.33 (R2.66 incl. VAT) on buy leg } \\
\text { and R9.43 (R10.75 incl. VAT) on sell side leg }\end{array}$ \\
\hline
\end{tabular}




\begin{tabular}{|c|c|c|c|c|}
\hline $\begin{array}{l}\text { Market } \\
\text { North America }\end{array}$ & Legal Origin & Capital Gains Tax & Other Taxes and Fees & Commission \\
\hline Canada & Common Law & Exempt & None & $\begin{array}{l}\text { Toronto Stock Exchange: Charged using a value- } \\
\text { based model: Active side is charged } 1 / 60 \text { th of } 1 \% \text { of } \\
\text { the value of the trade to a maximum of } \$ 50 \text {. }\end{array}$ \\
\hline \multirow[t]{2}{*}{ United States } & Common Law & $\begin{array}{l}\text { Capital gains are taxed as ordinary } \\
\text { income (up to a } 39.6 \% \text { marginal rate). } \\
\text { Long term capital gains are taxed up to } \\
\text { a } 20 \% \text { marginal rate. }\end{array}$ & $\begin{array}{l}\text { US Citizens \& Residents : } \\
\text { Dividends, interest and short term } \\
\text { assets (assets held less than } 12 \text { months). }\end{array}$ & $\begin{array}{l}\text { As NYSE Euronext Belgium (integrated trading } \\
\text { platform and fee structure reflects this) }\end{array}$ \\
\hline & & $\begin{array}{l}\text { Capital gains generally are not subject } \\
\text { to income tax }\end{array}$ & $\begin{array}{l}\text { Non-Resident Aliens : } \\
\text { Dividend and interest income is subject } \\
\text { to a } 30 \% \text { withholding tax. This tax } \\
\text { may be reduced of eliminated if the } \\
\text { non-resident is entitled to the benefit of } \\
\text { a tax treaty. }\end{array}$ & \\
\hline \multicolumn{5}{|l|}{ Australasia } \\
\hline Australia & Common Law & $\begin{array}{l}\text { Capital Gains Tax } \\
\text { - Shares held < } 12 \text { months, } 0-47 \% \\
\text { - Shares held > } 12 \text { months, } 0-23.5 \% \\
\text { Non-residents (Individuals) Income } \\
\text { Tax Dividends : } \\
\text { - } 100 \% \text { franked - } 0 \% \\
\text { - unfranked } \\
\text { - Tax treaty, } 0-15 \% \text { Witholding Tax } \\
\text { - No treaty, 30\% Witholding Tax }\end{array}$ & $\begin{array}{l}\text { Residents (Individuals) } \\
\text { Income Tax Dividends: } \\
\text { - franked } 0-17 \% \\
\text { - unfranked } 0-47 \%\end{array}$ & None \\
\hline New Zealand & Common Law & $\begin{array}{l}\text { Residents : } \\
\text { Dividend: } 33 \% \\
\text { Non-Residents : } \\
\text { Dividends : } 30 \% \text { or where double tax } \\
\text { treaty exists } 15 \%\end{array}$ & None & None \\
\hline \multicolumn{5}{|l|}{ Latin America } \\
\hline Argentina & French Civil Code & Exempt & None & $\begin{array}{l}\text { Stock Exchange Fee } 0,0351 \% \\
\text { Mercado de Valores de Buenos Aires (MV) Fee : } \\
0,06 \%\end{array}$ \\
\hline Brazil & French Civil Code & Exempt & None & $\begin{array}{l}\text { Commission: } 0.5-2 \% \text { max. based on volume; fully } \\
\text { negotiable } \\
\text { Transaction Fee: } 0.025 \% \\
\text { Clearing Fee: } 0.025 \%\end{array}$ \\
\hline Chile & French Civil Code & $15 \%$ & $\begin{array}{l}\text { Value Added Tax: } 18 \% \text { on commission } \\
\text { \& Exchange fees }\end{array}$ & $\begin{array}{l}\text { Commission: } 0.35 \%-1 \% \text {; negotiable. } \\
\text { Exchange Fees: Up to } 0.5 \% \text { based on volume }\end{array}$ \\
\hline
\end{tabular}




\begin{tabular}{|c|c|c|c|c|}
\hline Market & Legal Origin & Capital Gains Tax & Other Taxes and Fees & Commission \\
\hline Colombia & French Civil Code & Exempt & None & $\begin{array}{l}\text { Transaction Fee (percentage on trading value): } \\
0.0250 \%(0-500 \mathrm{MM}) \\
0.02500 \%(500.1-1000 \mathrm{MM}) \\
0.0150 \%(>1000.1 \mathrm{MM})\end{array}$ \\
\hline Jamaica & Common Law & Exempt & None & \\
\hline Mexico & French Civil Code & Exempt & None & $\begin{array}{l}\text { Commission: } 1-1.7 \% \text { based on value; banks and } \\
\text { institutions pay flat } 0.85 \% \text {; negotiable }\end{array}$ \\
\hline Peru & French Civil Code & Exempt & $\begin{array}{l}\text { Dividends: } 4.1 \% \text {. Domestic } \\
\text { institutional investors are exempt from } \\
\text { this tax } \\
\text { VAT: } 18 \%\end{array}$ & $\begin{array}{l}\text { Fees for exchange based transactions: } 0.0825 \% \\
\text { trading fee, } 0.0075 \% \text { guaranty fund }\end{array}$ \\
\hline $\begin{array}{l}\text { Venezuela } \\
\text { Asia Developed }\end{array}$ & French Civil Code & $30 \%$ & None & Variable; determined by brokers \\
\hline Japan & German Civil Code & $\begin{array}{l}\text { Individual Capital Gain } \\
\text { <Until Dec 31, 2009> } \\
10 \% \text { tax withheld at source } \\
\text { (Declaration of tax not required) } \\
7 \% \text { Income Tax, 3\% Inhabitant Tax }\end{array}$ & $\begin{array}{l}\text { Dividend } \\
<\text { Until March 31, 2010> } \\
10 \% \text { tax withheld at source } \\
\text { (Declaration of tax not required) } \\
7 \% \text { Income Tax, } 3 \% \text { Inhabitant Tax } \\
\text { Corporation } \\
50 \% \text { of the total amount of dividend } \\
\text { received is not taxable }\end{array}$ & $\begin{array}{l}\text { (1) Out of the total amount comprising the monthly } \\
\text { sales value and purchase value within the market of } \\
\text { auction trading and closing price trading } \\
¥ 20 \text { billion or less: } 0 \\
¥ 20 \text { billion }<\text { MTV* } 1 \leq ¥ 200 \text { billion: Standard rate*2 } \\
\text { x } 0.9 \\
¥ 200 \text { billion }<M T V \leq ¥ 1 \text { trillion: Standard rate } \\
¥ 1 \text { trillion }<\text { MTV } \leq ¥ 1.5 \text { trillion: Standard rate x } 0.8 \\
\text { More than } ¥ 1.5 \text { trillion: Standard rate x } 0.7 \\
* 1 \text { MTV:Monthly trading value } \\
* 2 \text { Standard rate is calculated using formula below: } \\
\{¥ 20 \text { trillion } x 0.000029+(\text { MTV }-¥ 20 \text { trillion) } \mathrm{x} \\
0.000020\} \div \text { MTV" } \\
(2) 0.04 / 10,000 \text { of the total amount comprising the } \\
\text { sales value and purchase value within the market of } \\
\text { trading other than auction trading and closing price } \\
\text { trading }\end{array}$ \\
\hline Singapore & Common Law & Exempt & $\begin{array}{l}\text { Prevailing Goods and Services tax } \\
\text { (GST) on brokerage and clearing fees }\end{array}$ & $\begin{array}{l}\text { Exchange levied Clearing Fees } \\
\text { Cash : } 4 \text { basis pts (cap of } \$ \$ 600 \text { ) }\end{array}$ \\
\hline $\begin{array}{l}\text { Asia Emerging } \\
\text { China Shenzen }\end{array}$ & German Civil Code & Exempt & $0.1 \%$ stamp duty (only for sell-side) & $\begin{array}{l}\text { floating commission is imposed for both buyers and } \\
\text { sellers, with maximum } 0.3 \% \text { of traded value }\end{array}$ \\
\hline China Shanghai & German Civil Code & Exempt & $\begin{array}{l}0.3 \% \text { stamp duty (levied on " } A \text { " shares) } \\
0.1 \% \text { Transfer fee }\end{array}$ & $\begin{array}{l}\text { A-share: Handling fee of } 0.011 \% \text {, securities } \\
\text { management fee of } 0.004 \%, . \\
\text { B-share: } 0.026 \% \text { Handling fee, } 0.004 \% \text { securities } \\
\text { management fee }\end{array}$ \\
\hline
\end{tabular}




\begin{tabular}{|c|c|c|c|c|}
\hline Market & Legal Origin & Capital Gains Tax & Other Taxes and Fees & Commission \\
\hline Hong Kong & Common Law & Exempt & Stamp duty at a rate of $0.1 \%$ & $\begin{array}{l}\text { Trading fee of } 0.005 \% \text {, transaction fee of } 0.004 \% \text {, } \\
\text { investor compensation guaranty fee of } 0.002 \%\end{array}$ \\
\hline Indonesia & French Civil Code & Exempt & $\begin{array}{l}\text { Stamp Duty: Rp. } 1,000 / \text { transaction } \\
\text { Value Added Tax: } 10 \% \text { on commission }\end{array}$ & $\begin{array}{l}\text { Commission: } 1 \% \text {. Buyer and seller. } \\
\text { Stock Exchange Levy: } 0.1 \%\end{array}$ \\
\hline Malaysia & Common Law & Exempt & $\begin{array}{l}\text { RM1.00 for RM } 1000.00 \text { or fractional } \\
\text { part of value of securities (payable by } \\
\text { both buyer and seller), and effective } 17 \\
\text { March } 2003 \text {, the stamp duty shall be } \\
\text { remitted to the maximum of RM200 }\end{array}$ & $\begin{array}{l}\text { Commission and Fees } \\
\text { Commission: } 1 \% \text {; } 5 \text { ringgit minimum; buyer \& seller } \\
\text { Clearing Fee: } 0.05 \% \\
\text { Deposit Fee: } 10 \text { ringgit per scrip (applies to investors } \\
\text { using Central Depository System) } \\
\text { Withdrawal Fee: } 15 \text { ringgit per } 1,000 \text { shares (applies } \\
\text { to investors using Central Depository System) } \\
\text { Transfer Fee: } 10 \text { ringgit (applies to investors using } \\
\text { Central Depository System) } \\
\text { Account Opening Fee: } 10 \text { ringgit (applies to } \\
\text { investors using Central Depository System) } \\
\text { Statement Fee: } 1 \text { ringgit (applies to investors using } \\
\text { Central Depository System) }\end{array}$ \\
\hline Pakistan & Common Law & Exempt & & \\
\hline Philippines & French Civil Code & $\begin{array}{l}\text { Capital Gains Tax of - } \\
5 \% \text { - transactions }<\mathrm{P} 100,000 \\
10 \% \text { - transactions }>\mathrm{P} 100,000\end{array}$ & $\begin{array}{l}\text { Dividend withholding tax. } \\
\text { Resident individuals: } 10 \% \text {; Resident } \\
\text { corporations: None; Non-residents: } \\
20 \% \\
\text { Stock Transaction Tax }-1 / 2 \text { of } 1 \% \text { of } \\
\text { the total transaction value }\end{array}$ & $\begin{array}{l}\text { Regular trades : } 0.00005 \% \text { for every transaction } \\
\text { executed } \\
\text { Negotiated trades : } 1 / 200 \text { of } 1 \% \text { for every contract } \\
\text { executed }\end{array}$ \\
\hline South Korea & German Civil Code & $10-25 \%$ & & \\
\hline Sri Lanka & Common Law & $15 \%$ & None & $\begin{array}{l}\text { Transactions < R 1M: } 1.425 \% \text { (Broker : } 1.00 \% \text {; } \\
\text { SEC: } 0.09 \% \text {; Exchange: } 0.105 \% \text {; CDS : } 0.03 \% \text {; } \\
\text { Gvmt Tax : } 0.2 \% \text { ) } \\
\text { Transactions > R 1M: } 1.225 \% \text { (Broker : } 0.80 \% \text {; } \\
\text { SEC: } 0.09 \% \text {; Exchange: } 0.105 \% \text {; CDS : } 0.03 \% \text {; } \\
\text { Gvmt Tax : } 0.2 \% \text { ) }\end{array}$ \\
\hline Taiwan & Common Law & Exempt & $\begin{array}{l}\text { Non-residents, } \\
\text { tax rate on dividends is } 30 \% \text {, and } 25 \% \\
\text { for companies with no fixed business } \\
\text { place in Taiwan, } \\
\text { and } 20 \% \text { for investments approved } \\
\text { under the statute of investment for } \\
\text { foreign nationals }\end{array}$ & $\begin{array}{l}\text { TSEC levies } 0.0065 \% \text { on the trading value of } \\
\text { brokers for handling fee. As for the transaction tax, } \\
0.3 \% \text { levied from the sales of shares }\end{array}$ \\
\hline
\end{tabular}




\begin{tabular}{|c|c|c|c|c|}
\hline Market & Legal Origin & Capital Gains Tax & Other Taxes and Fees & Commission \\
\hline Thailand & Common Law & $\begin{array}{l}\text { Tax-free for individual investors, but } \\
15 \% \text { witholding tax for foreign juristic } \\
\text { investors }\end{array}$ & $10 \%$ witholding tax & $\begin{array}{l}\text { Admission fee: } 0.040 \% \text { of securities trading value } \\
\text { every month, throughout the period of } 3 \text { years } \\
\text { Trading fee: THB } 50,000 \text { plus } 0.005 \% \text { of monthly } \\
\text { securities trading value }\end{array}$ \\
\hline Vietnam & French Civil Code & & & \\
\hline Middle East & & & & \\
\hline Abu Dhabi & Common Law & Exempt & None & $\begin{array}{l}\text { Overall trading fee of } 0.275 \% \text {, made up from } 0.15 \% \\
\text { brokers commission, } 0.05 \% \text { SEC fee, } 0.05 \% \text { CSD } \\
\text { fee, } 0.025 \% \text { authority fee. }\end{array}$ \\
\hline Dubai & Common Law & Exempt & None & \\
\hline Israel & Common Law & Exempt & $20 \%$ witholding tax & $\begin{array}{l}\text { Trading fee: } 0.0035 \% \text {, minimum - } 1.07 \text { NIS, } \\
\text { maximum - } 107 \text { NIS }\end{array}$ \\
\hline Jordan & French Civil Code & Exempt & None & $\begin{array}{l}\text { Floor-based trading: Total fee: } 0.0014 \% \\
\text { (SEC:0.0005\%; Exchange: } 0.0005 \% \text {; CSD: } \\
0.0004 \% \text { ) } \\
\text { Off Floor-based trading: Total fee: } 0.002 \% \text { (CSD: } \\
0.002 \% \text { ) } \\
\text { Brokerage fees for shares fall between } 0.004 \% \text { - } \\
0.006 \%\end{array}$ \\
\hline Kuwait & Common Law & Exempt & None & $\begin{array}{l}\text { Transactions fees calculated: } \\
\text { 1. Less than } 50,000 \mathrm{KD} \text { transactions, commission is } \\
\text { 1.25 KD for each KD } 1000 \text {. } \\
\text { 2. More than } 50,000 \mathrm{KD} \text { transactions, the } \\
\text { commission is } 0.001 \%\end{array}$ \\
\hline Oman & French Civil Code & Exempt & None & $\begin{array}{l}\text { Trading fee of } 0.1 \% \text { for orders }<\text { RO } 250,000 \text { and } \\
0.05 \%>\text { RO } 250,000 \text {. } \\
\text { Broker commissions fixed between } 0.4 \% \text { and } 0.75 \% \text {, } \\
\text { where exchange takes } 20 \% \text { of brokerage fees levied. }\end{array}$ \\
\hline Qatar & Common Law & Exempt & None & \\
\hline Saudi Arabia & Common Law & Exempt & None & $\begin{array}{l}\text { Brokerage commission max is } 0.0012 \% \text { of the trade } \\
\text { value. Min commission imposed will be SR } 12.00 \\
\text { for any executed order equal or less than SR } 10,000\end{array}$ \\
\hline Turkey & French Civil Code & Exempt & None & Exchange transaction fee: $0.00001 \%$ \\
\hline
\end{tabular}


Appendix 2 Summary of Secondary Market regulations and fees for selected countries

\begin{tabular}{|c|c|c|c|c|c|c|c|c|}
\hline \multicolumn{9}{|c|}{ Panel 1: Results for Austria (2001M07 - 2009M05) } \\
\hline & Price & Volatility & Volume & MV & Amihud & Liu & Turnover & Bid Ask Spread \\
\hline Price & 1.00000 & & & & & & & \\
\hline Volatility & -0.18383 & 1.00000 & & & & & & \\
\hline Volume & -0.22935 & -0.00045 & 1.00000 & & & & & \\
\hline MV & 0.46117 & -0.22863 & 0.43306 & 1.00000 & & & & \\
\hline Amihud & -0.30567 & 0.35372 & -0.62597 & -0.68049 & 1.00000 & & & \\
\hline Liu & 0.06697 & 0.05832 & -0.79852 & -0.30395 & 0.68353 & 1.00000 & & \\
\hline Turnover & -0.13974 & 0.14296 & 0.69385 & -0.03933 & -0.41389 & -0.70098 & 1.00000 & \\
\hline Bid Ask Spread & -0.19093 & 0.30411 & -0.53225 & -0.50802 & 0.69982 & 0.60560 & -0.30304 & 1.00000 \\
\hline \multicolumn{9}{|c|}{ Panel 2: Results for Belgium (2005M04 - 2009M05) } \\
\hline & Price & Volatility & Volume & MV & Amihud & Liu & Turnover & Bid Ask Spread \\
\hline Price & 1.00000 & & & & & & & \\
\hline Volatility & -0.35569 & 1.00000 & & & & & & \\
\hline Volume & -0.53341 & 0.13895 & 1.00000 & & & & & \\
\hline MV & 0.19881 & -0.26879 & 0.57050 & 1.00000 & & & & \\
\hline Amihud & 0.02182 & 0.20648 & -0.77648 & -0.81391 & 1.00000 & & & \\
\hline Liu & 0.30564 & -0.34502 & -0.32146 & 0.26793 & 0.10853 & 1.00000 & & \\
\hline Turnover & -0.32898 & 0.36694 & 0.34773 & -0.26156 & -0.11162 & -0.97578 & 1.00000 & \\
\hline Bid Ask Spread & -0.12288 & 0.28995 & -0.56317 & -0.62106 & 0.80322 & 0.15715 & -0.15024 & 1.00000 \\
\hline \multicolumn{9}{|c|}{ Panel 3: Results for Denmark (2001M07 - 2009M05) } \\
\hline & Price & Volatility & Volume & MV & Amihud & Liu & Turnover & Bid Ask Spread \\
\hline Price & 1.00000 & & & & & & & \\
\hline Volatility & -0.21754 & 1.00000 & & & & & & \\
\hline Volume & -0.41251 & 0.07659 & 1.00000 & & & & & \\
\hline MV & 0.35453 & -0.15211 & 0.49918 & 1.00000 & & & & \\
\hline Amihud & -0.23174 & 0.15912 & -0.66386 & -0.85392 & 1.00000 & & & \\
\hline Liu & 0.13189 & -0.20978 & -0.62369 & -0.20737 & 0.54542 & 1.00000 & & \\
\hline Turnover & -0.30604 & 0.21718 & 0.49541 & -0.09037 & -0.30966 & -0.78312 & 1.00000 & \\
\hline Bid Ask Spread & -0.24254 & 0.21265 & -0.60766 & -0.74305 & 0.87985 & 0.54228 & -0.31376 & 1.00000 \\
\hline \multicolumn{9}{|c|}{ Panel 4: Results for Finland (2008M12 - 2009M05) } \\
\hline & Price & Volatility & Volume & MV & Amihud & Liu & Turnover & Bid Ask Spread \\
\hline Price & 1.00000 & & & & & & & \\
\hline Volatility & -0.01825 & 1.00000 & & & & & & \\
\hline Volume & 0.08284 & 0.31704 & 1.00000 & & & & & \\
\hline MV & 0.50926 & 0.19872 & 0.74272 & 1.00000 & & & & \\
\hline Amihud & -0.41554 & -0.18397 & -0.89874 & -0.85869 & 1.00000 & & & \\
\hline Liu & -0.21095 & -0.34846 & -0.80707 & -0.48479 & 0.77572 & 1.00000 & & \\
\hline Turnover & 0.20190 & 0.33890 & 0.79925 & 0.45516 & -0.76014 & -0.99077 & 1.00000 & \\
\hline Bid Ask Spread & -0.46883 & -0.15252 & -0.83215 & -0.80515 & 0.94351 & 0.78135 & -0.76897 & 1.00000 \\
\hline
\end{tabular}




\begin{tabular}{|c|c|c|c|c|c|c|c|c|}
\hline \multicolumn{9}{|c|}{ Panel 5: Results for France (2001M07 - 2009M05) } \\
\hline & Price & Volatility & Volume & MV & Amihud & Liu & Turnover & Bid Ask Spread \\
\hline Price & 1.00000 & & & & & & & \\
\hline Volatility & -0.19839 & 1.00000 & & & & & & \\
\hline Volume & -0.34858 & 0.13317 & 1.00000 & & & & & \\
\hline MV & 0.18611 & -0.05373 & 0.59012 & 1.00000 & & & & \\
\hline Amihud & -0.28672 & 0.25319 & -0.63963 & -0.72441 & 1.00000 & & & \\
\hline Liu & 0.00402 & -0.14044 & -0.14283 & 0.43649 & 0.08013 & 1.00000 & & \\
\hline Turnover & -0.00440 & 0.14214 & 0.14254 & -0.43724 & -0.07899 & -0.99932 & 1.00000 & \\
\hline Bid Ask Spread & -0.22020 & 0.17284 & -0.38479 & -0.46378 & 0.62070 & 0.09613 & -0.09518 & 1.00000 \\
\hline \multicolumn{9}{|c|}{ Panel 6: Results for Germany (2008M12 - 2009M05) } \\
\hline & Price & Volatility & Volume & MV & Amihud & Liu & Turnover & Bid Ask Spread \\
\hline Price & 1.00000 & & & & & & & \\
\hline Volatility & -0.44975 & 1.00000 & & & & & & \\
\hline Volume & -0.34358 & 0.44179 & 1.00000 & & & & & \\
\hline MV & 0.63910 & -0.28853 & 0.26917 & 1.00000 & & & & \\
\hline Amihud & -0.36133 & 0.06416 & -0.64070 & -0.75984 & 1.00000 & & & \\
\hline Liu & 0.46155 & -0.59737 & -0.70152 & 0.28908 & 0.25333 & 1.00000 & & \\
\hline Turnover & -0.54821 & 0.61751 & 0.60493 & -0.45288 & -0.11770 & -0.94611 & 1.00000 & \\
\hline Bid Ask Spread & -0.50376 & 0.24704 & -0.36972 & -0.85551 & 0.82176 & -0.05990 & 0.20538 & 1.00000 \\
\hline \multicolumn{9}{|c|}{ Panel 7: Results for Iceland (2006M07 - 2009M05) } \\
\hline & Price & Volatility & Volume & MV & Amihud & Liu & Turnover & Bid Ask Spread \\
\hline Price & 1.00000 & & & & & & & \\
\hline Volatility & 0.24810 & 1.00000 & & & & & & \\
\hline Volume & -0.70136 & -0.17034 & 1.00000 & & & & & \\
\hline MV & -0.25449 & -0.25122 & 0.61408 & 1.00000 & & & & \\
\hline Amihud & 0.11653 & 0.22891 & -0.52014 & -0.64762 & 1.00000 & & & \\
\hline $\mathrm{Liu}$ & -0.29034 & -0.18728 & -0.22122 & -0.37129 & 0.47673 & 1.00000 & & \\
\hline Turnover & 0.08463 & 0.20442 & 0.40184 & 0.18980 & -0.35748 & -0.62224 & 1.00000 & \\
\hline Bid Ask Spread & 0.24782 & 0.23007 & -0.40639 & -0.21163 & 0.35619 & 0.26537 & -0.32687 & 1.00000 \\
\hline \multicolumn{9}{|c|}{ Panel 8: Results for Ireland (2005M03 - 2009M05) } \\
\hline & Price & Volatility & Volume & MV & Amihud & Liu & Turnover & Bid Ask Spread \\
\hline Price & 1.00000 & & & & & & & \\
\hline Volatility & -0.58176 & 1.00000 & & & & & & \\
\hline Volume & 0.21534 & -0.04911 & 1.00000 & & & & & \\
\hline MV & 0.78788 & -0.40261 & 0.60007 & 1.00000 & & & & \\
\hline Amihud & -0.77020 & 0.47474 & -0.71247 & -0.88844 & 1.00000 & & & \\
\hline Liu & -0.47731 & 0.23853 & -0.82306 & -0.66366 & 0.80849 & 1.00000 & & \\
\hline Turnover & 0.33790 & -0.17080 & 0.70840 & 0.33458 & -0.61542 & -0.75803 & 1.00000 & \\
\hline Bid Ask Spread & -0.75346 & 0.52230 & -0.66527 & -0.83380 & 0.92023 & 0.81536 & -0.60469 & 1.00000 \\
\hline
\end{tabular}




\begin{tabular}{|c|c|c|c|c|c|c|c|c|}
\hline \multicolumn{9}{|c|}{ Panel 9: Results for Italy (2005M04 - 2009M05) } \\
\hline & Price & Volatility & Volume & MV & Amihud & Liu & Turnover & Bid Ask Spread \\
\hline Price & 1.00000 & & & & & & & \\
\hline Volatility & -0.06535 & 1.00000 & & & & & & \\
\hline Volume & -0.50733 & 0.35116 & 1.00000 & & & & & \\
\hline MV & 0.08096 & 0.00280 & 0.51239 & 1.00000 & & & & \\
\hline Amihud & -0.06674 & -0.14733 & -0.72599 & -0.70482 & 1.00000 & & & \\
\hline Liu & -0.05795 & -0.47572 & -0.48698 & 0.08644 & 0.48670 & 1.00000 & & \\
\hline Turnover & 0.05421 & 0.49357 & 0.48272 & -0.11561 & -0.47525 & -0.97354 & 1.00000 & \\
\hline Bid Ask Spread & -0.02889 & -0.10216 & -0.61401 & -0.59310 & 0.78910 & 0.38832 & -0.37681 & 1.00000 \\
\hline \multicolumn{9}{|c|}{ Panel 10: Results for Luxembourg (2005M03 - 2009M05) } \\
\hline & Price & Volatility & Volume & MV & Amihud & Liu & Turnover & Bid Ask Spread \\
\hline Price & 1.00000 & & & & & & & \\
\hline Volatility & -0.29560 & 1.00000 & & & & & & \\
\hline Volume & -0.48791 & -0.01346 & 1.00000 & & & & & \\
\hline MV & -0.28187 & 0.36813 & 0.07418 & 1.00000 & & & & \\
\hline Amihud & -0.05549 & 0.32857 & -0.47857 & -0.02665 & 1.00000 & & & \\
\hline Liu & 0.04368 & 0.25934 & -0.41868 & 0.73407 & 0.28956 & 1.00000 & & \\
\hline Turnover & 0.44258 & -0.40467 & -0.03132 & -0.89011 & -0.16951 & -0.71401 & 1.00000 & \\
\hline Bid Ask Spread & -0.27225 & 0.38242 & 0.06374 & 0.16291 & 0.23846 & 0.11731 & -0.19560 & 1.00000 \\
\hline \multicolumn{9}{|c|}{ Panel 11: Results for Netherlands (2005M04 - 2009M05) } \\
\hline & Price & Volatility & Volume & MV & Amihud & Liu & Turnover & Bid Ask Spread \\
\hline Price & 1.00000 & & & & & & & \\
\hline Volatility & -0.30928 & 1.00000 & & & & & & \\
\hline Volume & -0.00227 & 0.03783 & 1.00000 & & & & & \\
\hline MV & $\mathbf{0 . 5 3 3 7 7}$ & -0.22521 & 0.66361 & 1.00000 & & & & \\
\hline Amihud & -0.39719 & 0.21337 & -0.86203 & -0.85199 & 1.00000 & & & \\
\hline Liu & 0.01613 & -0.15892 & -0.83130 & -0.38803 & 0.71010 & 1.00000 & & \\
\hline Turnover & -0.02361 & 0.17386 & 0.80308 & 0.31611 & -0.67195 & -0.95852 & 1.00000 & \\
\hline Bid Ask Spread & -0.35168 & 0.22771 & -0.82642 & -0.79757 & 0.94676 & 0.69443 & -0.64800 & 1.00000 \\
\hline \multicolumn{9}{|c|}{ Panel 12: Results for Norway $(2005 \mathrm{M03}-2009 \mathrm{M05})$} \\
\hline & Price & Volatility & Volume & MV & Amihud & Liu & Turnover & Bid Ask Spread \\
\hline Price & 1.00000 & & & & & & & \\
\hline Volatility & -0.23473 & 1.00000 & & & & & & \\
\hline Volume & -0.01628 & 0.24420 & 1.00000 & & & & & \\
\hline MV & 0.65545 & -0.12438 & 0.58266 & 1.00000 & & & & \\
\hline Amihud & -0.45283 & -0.01698 & -0.83572 & -0.83927 & 1.00000 & & & \\
\hline Liu & -0.02240 & -0.38010 & -0.78805 & -0.31185 & 0.67860 & 1.00000 & & \\
\hline Turnover & -0.02430 & 0.40481 & 0.78149 & 0.25056 & -0.63441 & -0.95663 & 1.00000 & \\
\hline Bid Ask Spread & -0.45190 & -0.01565 & -0.77208 & -0.78159 & 0.92778 & 0.67040 & -0.62361 & 1.00000 \\
\hline
\end{tabular}




\begin{tabular}{|c|c|c|c|c|c|c|c|c|}
\hline \multicolumn{9}{|c|}{ Panel 13: Results for Portugal (2005M04 - 2009M05) } \\
\hline & Price & Volatility & Volume & MV & Amihud & Liu & Turnover & Bid Ask Spread \\
\hline Price & 1.00000 & & & & & & & \\
\hline Volatility & -0.36955 & 1.00000 & & & & & & \\
\hline Volume & 0.22506 & -0.14362 & 1.00000 & & & & & \\
\hline MV & 0.59949 & -0.36216 & 0.78715 & 1.00000 & & & & \\
\hline Amihud & -0.52622 & 0.37220 & -0.89776 & -0.90164 & 1.00000 & & & \\
\hline Liu & -0.15953 & 0.08496 & -0.82916 & -0.52486 & 0.74841 & 1.00000 & & \\
\hline Turnover & 0.05489 & 0.04537 & 0.76665 & 0.34632 & -0.62102 & -0.87139 & 1.00000 & \\
\hline Bid Ask Spread & -0.58591 & 0.41456 & -0.80204 & -0.84157 & 0.92577 & 0.69774 & -0.56062 & 1.00000 \\
\hline \multicolumn{9}{|c|}{ Panel 14: Results for Slovenia (2005M03 - 2009M05) } \\
\hline & Price & Volatility & Volume & MV & Amihud & Liu & Turnover & Bid Ask Spread \\
\hline Price & 1.00000 & & & & & & & \\
\hline Volatility & -0.03470 & 1.00000 & & & & & & \\
\hline Volume & -0.59646 & -0.17347 & 1.00000 & & & & & \\
\hline MV & 0.62768 & -0.17463 & -0.19754 & 1.00000 & & & & \\
\hline Amihud & -0.36515 & 0.40860 & -0.34306 & -0.53181 & 1.00000 & & & \\
\hline Liu & 0.28766 & 0.15466 & -0.76836 & 0.11142 & 0.43964 & 1.00000 & & \\
\hline Turnover & -0.26147 & -0.12561 & 0.74435 & -0.21308 & -0.39720 & -0.87061 & 1.00000 & \\
\hline Bid Ask Spread & -0.10338 & 0.41716 & -0.53947 & -0.34625 & 0.77030 & 0.58787 & -0.52401 & 1.00000 \\
\hline \multicolumn{9}{|c|}{ Panel 15: Results for Spain (2008M12 - 2009M05) } \\
\hline & Price & Volatility & Volume & MV & Amihud & Liu & Turnover & Bid Ask Spread \\
\hline Price & 1.00000 & & & & & & & \\
\hline Volatility & -0.32429 & 1.00000 & & & & & & \\
\hline Volume & -0.18951 & 0.20798 & 1.00000 & & & & & \\
\hline MV & 0.51623 & -0.19450 & 0.53527 & 1.00000 & & & & \\
\hline Amihud & -0.32840 & 0.14924 & -0.78619 & -0.83274 & 1.00000 & & & \\
\hline $\mathrm{Liu}$ & 0.13805 & -0.27757 & -0.86004 & -0.29488 & 0.66563 & 1.00000 & & \\
\hline Turnover & -0.14796 & 0.29526 & 0.84815 & 0.24985 & -0.63491 & -0.97200 & 1.00000 & \\
\hline Bid Ask Spread & -0.42107 & 0.25789 & -0.64329 & -0.84279 & 0.92498 & 0.52344 & -0.48205 & 1.00000 \\
\hline \multicolumn{9}{|c|}{ Panel 16: Results for Sweden (2001M07 - 2009M05) } \\
\hline & Price & Volatility & Volume & MV & Amihud & Liu & Turnover & Bid Ask Spread \\
\hline Price & 1.00000 & & & & & & & \\
\hline Volatility & -0.26886 & 1.00000 & & & & & & \\
\hline Volume & 0.09005 & -0.02759 & 1.00000 & & & & & \\
\hline MV & 0.54848 & -0.15618 & 0.65134 & 1.00000 & & & & \\
\hline Amihud & -0.42887 & 0.22364 & -0.89648 & -0.79755 & 1.00000 & & & \\
\hline Liu & 0.01865 & -0.04033 & -0.67491 & -0.08823 & 0.55536 & 1.00000 & & \\
\hline Turnover & -0.07493 & 0.05597 & 0.64581 & 0.01618 & -0.50791 & -0.96086 & 1.00000 & \\
\hline Bid Ask Spread & -0.44459 & 0.25026 & -0.75485 & -0.65746 & 0.88354 & 0.54994 & -0.50217 & 1.00000 \\
\hline
\end{tabular}




\begin{tabular}{|c|c|c|c|c|c|c|c|c|}
\hline \multicolumn{9}{|c|}{ Panel 17: Results for Switzerland (2006M12 - 2009M05) } \\
\hline & Price & Volatility & Volume & MV & Amihud & Liu & Turnover & Bid Ask Spread \\
\hline Price & 1.00000 & & & & & & & \\
\hline Volatility & -0.34084 & 1.00000 & & & & & & \\
\hline Volume & -0.74437 & 0.14407 & 1.00000 & & & & & \\
\hline MV & 0.04395 & -0.28344 & 0.51155 & 1.00000 & & & & \\
\hline Amihud & 0.05614 & 0.35168 & -0.63423 & -0.86235 & 1.00000 & & & \\
\hline Liu & 0.40402 & -0.46225 & -0.35503 & 0.31585 & 0.01538 & 1.00000 & & \\
\hline Turnover & -0.40629 & 0.46554 & 0.35570 & -0.31718 & -0.01351 & -0.99798 & 1.00000 & \\
\hline \multicolumn{9}{|c|}{ Bid Ask Spread } \\
\hline \multicolumn{9}{|c|}{ Panel 18: Results for UK (2003M07 - 2009M05) } \\
\hline & Price & Volatility & Volume & MV & Amihud & Liu & Turnover & Bid Ask Spread \\
\hline Price & 1.00000 & & & & & & & \\
\hline Volatility & -0.11351 & 1.00000 & & & & & & \\
\hline Volume & -0.36899 & 0.01789 & 1.00000 & & & & & \\
\hline MV & 0.31961 & -0.21032 & 0.60481 & 1.00000 & & & & \\
\hline Amihud & -0.32445 & 0.29825 & -0.66578 & -0.87273 & 1.00000 & & & \\
\hline Liu & 0.16241 & -0.29080 & -0.21909 & 0.29258 & 0.00434 & 1.00000 & & \\
\hline Turnover & -0.16338 & 0.29176 & 0.21907 & -0.29397 & -0.00364 & -0.99855 & 1.00000 & \\
\hline Bid Ask Spread & -0.40031 & 0.31113 & -0.44853 & -0.69237 & $\mathbf{0 . 7 8 3 2 6}$ & -0.02355 & 0.02518 & 1.00000 \\
\hline \multicolumn{9}{|c|}{ Panel 19: Results for Cyprus (2005M03 - 2009M05) } \\
\hline & Price & Volatility & Volume & MV & Amihud & Liu & Turnover & Bid Ask Spread \\
\hline Price & 1.00000 & & & & & & & \\
\hline Volatility & -0.66804 & 1.00000 & & & & & & \\
\hline Volume & -0.01929 & 0.02540 & 1.00000 & & & & & \\
\hline MV & 0.61339 & -0.48458 & 0.44591 & 1.00000 & & & & \\
\hline Amihud & -0.58922 & 0.53054 & -0.55757 & -0.67866 & 1.00000 & & & \\
\hline Liu & -0.19735 & 0.13072 & -0.85520 & -0.57602 & 0.63150 & 1.00000 & & \\
\hline Turnover & -0.08780 & 0.13748 & 0.55970 & -0.22414 & -0.27439 & -0.34890 & 1.00000 & \\
\hline Bid Ask Spread & -0.55706 & 0.51981 & -0.51409 & -0.71204 & 0.69621 & 0.68122 & -0.07065 & 1.00000 \\
\hline \multicolumn{9}{|c|}{ Panel 20: Results for Bulgaria (2005M07 - 2009M05) } \\
\hline & Price & Volatility & Volume & MV & Amihud & Liu & Turnover & Bid Ask Spread \\
\hline Price & 1.00000 & & & & & & & \\
\hline Volatility & 0.03286 & 1.00000 & & & & & & \\
\hline Volume & -0.39088 & -0.20203 & 1.00000 & & & & & \\
\hline MV & -0.02463 & -0.16490 & 0.52171 & 1.00000 & & & & \\
\hline Amihud & -0.12862 & 0.42670 & -0.45946 & -0.39098 & 1.00000 & & & \\
\hline Liu & 0.24213 & 0.28009 & -0.68808 & -0.18357 & 0.55471 & 1.00000 & & \\
\hline Turnover & 0.11642 & -0.02146 & 0.35684 & -0.23605 & -0.33648 & -0.47962 & 1.00000 & \\
\hline Bid Ask Spread & 0.06048 & 0.51671 & -0.54678 & -0.40149 & 0.67606 & 0.66315 & -0.17556 & 1.00000 \\
\hline
\end{tabular}




\begin{tabular}{|c|c|c|c|c|c|c|c|c|}
\hline \multicolumn{9}{|c|}{ Panel 21: Results for Czech Rep (2004M12 - 2009M05) } \\
\hline & Price & Volatility & Volume & MV & Amihud & Liu & Turnover & Bid Ask Spread \\
\hline Price & 1.00000 & & & & & & & \\
\hline Volatility & -0.28285 & 1.00000 & & & & & & \\
\hline Volume & 0.03898 & -0.18148 & 1.00000 & & & & & \\
\hline MV & 0.40679 & -0.30983 & 0.81808 & 1.00000 & & & & \\
\hline Amihud & -0.39136 & 0.31631 & -0.82998 & -0.94198 & 1.00000 & & & \\
\hline Liu & -0.28527 & 0.09555 & -0.36984 & -0.24109 & 0.37765 & 1.00000 & & \\
\hline Turnover & 0.26318 & -0.06142 & 0.35309 & 0.21429 & -0.35573 & -0.98179 & 1.00000 & \\
\hline Bid Ask Spread & -0.40141 & 0.29652 & -0.74766 & -0.86296 & 0.89868 & 0.41287 & -0.39065 & 1.00000 \\
\hline \multicolumn{9}{|c|}{ Panel 22: Results for Estonia (2005M07 - 2009M05) } \\
\hline & Price & Volatility & Volume & MV & Amihud & Liu & Turnover & Bid Ask Spread \\
\hline Price & 1.00000 & & & & & & & \\
\hline Volatility & -0.43907 & 1.00000 & & & & & & \\
\hline Volume & -0.17538 & -0.15257 & 1.00000 & & & & & \\
\hline MV & 0.48716 & -0.44149 & 0.39107 & 1.00000 & & & & \\
\hline Amihud & -0.26568 & 0.44415 & -0.72098 & -0.66732 & 1.00000 & & & \\
\hline Liu & 0.08581 & 0.12742 & -0.79142 & -0.24828 & 0.67907 & 1.00000 & & \\
\hline Turnover & -0.21866 & 0.02132 & 0.76319 & -0.04734 & -0.42256 & -0.71431 & 1.00000 & \\
\hline Bid Ask Spread & -0.34687 & 0.49457 & -0.64283 & -0.65278 & 0.84811 & 0.63659 & -0.37344 & 1.00000 \\
\hline \multicolumn{9}{|c|}{ Panel 23: Results for Hungary (2001M06 - 2009M05) } \\
\hline & Price & Volatility & Volume & MV & Amihud & Liu & Turnover & Bid Ask Spread \\
\hline Price & 1.00000 & & & & & & & \\
\hline Volatility & -0.26912 & 1.00000 & & & & & & \\
\hline Volume & -0.25622 & 0.11986 & 1.00000 & & & & & \\
\hline MV & 0.66006 & -0.26860 & 0.32740 & 1.00000 & & & & \\
\hline Amihud & -0.47625 & 0.16108 & -0.60470 & -0.77763 & 1.00000 & & & \\
\hline Liu & 0.18030 & -0.26347 & -0.53332 & 0.16610 & 0.30744 & 1.00000 & & \\
\hline Turnover & -0.25914 & 0.32607 & 0.51235 & -0.28008 & -0.21188 & -0.90526 & 1.00000 & \\
\hline Bid Ask Spread & -0.35584 & 0.27052 & -0.55275 & -0.68775 & 0.77116 & 0.20203 & -0.10785 & 1.00000 \\
\hline \multicolumn{9}{|c|}{ Panel 24: Results for Poland (2002M01 - 2009M05) } \\
\hline & Price & Volatility & Volume & MV & Amihud & Liu & Turnover & Bid Ask Spread \\
\hline Price & 1.00000 & & & & & & & \\
\hline Volatility & -0.13624 & 1.00000 & & & & & & \\
\hline Volume & -0.53014 & 0.19495 & 1.00000 & & & & & \\
\hline MV & 0.53226 & -0.17520 & 0.17077 & 1.00000 & & & & \\
\hline Amihud & -0.34097 & 0.11318 & -0.44642 & -0.79308 & 1.00000 & & & \\
\hline Liu & 0.42343 & -0.27332 & -0.44162 & 0.45840 & -0.05663 & 1.00000 & & \\
\hline Turnover & -0.52329 & 0.29979 & 0.40681 & -0.56366 & 0.16005 & -0.91852 & 1.00000 & \\
\hline Bid Ask Spread & -0.07621 & 0.09224 & -0.30111 & -0.39917 & 0.53768 & -0.00802 & 0.05013 & 1.00000 \\
\hline
\end{tabular}




\begin{tabular}{|c|c|c|c|c|c|c|c|c|}
\hline \multicolumn{9}{|c|}{ Panel 25: Results for Russia (2002M07 - 2009M05) } \\
\hline & Price & Volatility & Volume & MV & Amihud & Liu & Turnover & Bid Ask Spread \\
\hline Price & 1.00000 & & & & & & & \\
\hline Volatility & -0.35617 & 1.00000 & & & & & & \\
\hline Volume & -0.52800 & 0.24034 & 1.00000 & & & & & \\
\hline MV & 0.37496 & -0.17067 & 0.26334 & 1.00000 & & & & \\
\hline Amihud & -0.44736 & 0.37731 & -0.29723 & -0.59363 & 1.00000 & & & \\
\hline Liu & -0.03633 & -0.16109 & -0.60149 & -0.24956 & 0.47408 & 1.00000 & & \\
\hline Turnover & -0.13653 & 0.10876 & 0.19741 & -0.55807 & -0.05067 & -0.31745 & 1.00000 & \\
\hline Bid Ask Spread & -0.19266 & 0.17520 & -0.46575 & -0.49058 & 0.60706 & 0.66927 & -0.01904 & 1.00000 \\
\hline \multicolumn{9}{|c|}{ Panel 26: Results for Morocco (2005M01 - 2009M05) } \\
\hline & Price & Volatility & Volume & MV & Amihud & Liu & Turnover & Bid Ask Spread \\
\hline Price & 1.00000 & & & & & & & \\
\hline Volatility & 0.04954 & 1.00000 & & & & & & \\
\hline Volume & -0.45674 & -0.20350 & 1.00000 & & & & & \\
\hline MV & 0.46772 & -0.18187 & 0.25264 & 1.00000 & & & & \\
\hline Amihud & -0.04642 & 0.38324 & -0.60190 & -0.50792 & 1.00000 & & & \\
\hline Liu & 0.31612 & 0.16488 & -0.80695 & -0.22391 & 0.56701 & 1.00000 & & \\
\hline Turnover & -0.34751 & -0.01982 & 0.67754 & -0.26664 & -0.32378 & -0.57700 & 1.00000 & \\
\hline Bid Ask Spread & 0.22515 & 0.30677 & -0.60061 & -0.33681 & 0.53195 & 0.64009 & -0.28974 & 1.00000 \\
\hline \multicolumn{9}{|c|}{ Panel 27: Results for Egypt (2005M06 - 2009M05) } \\
\hline & Price & Volatility & Volume & MV & Amihud & Liu & Turnover & Bid Ask Spread \\
\hline Price & 1.00000 & & & & & & & \\
\hline Volatility & -0.28827 & 1.00000 & & & & & & \\
\hline Volume & -0.53152 & 0.23470 & 1.00000 & & & & & \\
\hline MV & 0.68384 & -0.34255 & -0.13586 & 1.00000 & & & & \\
\hline Amihud & -0.26461 & 0.16322 & -0.51157 & -0.51005 & 1.00000 & & & \\
\hline $\mathrm{Liu}$ & 0.36066 & -0.30972 & -0.66880 & 0.39383 & 0.34174 & 1.00000 & & \\
\hline Turnover & -0.44360 & 0.38594 & 0.67628 & -0.56312 & -0.24067 & -0.82521 & 1.00000 & \\
\hline Bid Ask Spread & 0.04461 & 0.12975 & -0.49012 & -0.24352 & 0.62009 & 0.29545 & -0.21777 & 1.00000 \\
\hline \multicolumn{9}{|c|}{ Panel 28: Results for South Africa $(2002 M 10$ - 2009M05) } \\
\hline & Price & Volatility & Volume & MV & Amihud & Liu & Turnover & Bid Ask Spread \\
\hline Price & 1.00000 & & & & & & & \\
\hline Volatility & 0.22447 & 1.00000 & & & & & & \\
\hline Volume & -0.27938 & 0.06284 & 1.00000 & & & & & \\
\hline MV & 0.50129 & 0.17220 & 0.45287 & 1.00000 & & & & \\
\hline Amihud & -0.38060 & -0.03339 & -0.67365 & -0.78039 & 1.00000 & & & \\
\hline Liu & 0.04390 & -0.14274 & -0.43301 & 0.14515 & 0.32853 & 1.00000 & & \\
\hline Turnover & -0.05080 & 0.13831 & 0.42640 & -0.16055 & -0.31802 & -0.98797 & 1.00000 & \\
\hline Bid Ask Spread & -0.34975 & -0.01030 & -0.54430 & -0.63787 & 0.78952 & 0.31126 & -0.30181 & 1.00000 \\
\hline
\end{tabular}




\begin{tabular}{|c|c|c|c|c|c|c|c|c|}
\hline \multicolumn{9}{|c|}{ Panel 29: Results for Canada (2006M12 - 2009M05) } \\
\hline & Price & Volatility & Volume & MV & Amihud & Liu & Turnover & Bid Ask Spread \\
\hline Price & 1.00000 & & & & & & & \\
\hline Volatility & -0.31830 & 1.00000 & & & & & & \\
\hline Volume & 0.03582 & 0.25099 & 1.00000 & & & & & \\
\hline MV & 0.67320 & -0.18486 & 0.60432 & 1.00000 & & & & \\
\hline Amihud & -0.61465 & 0.19758 & -0.71169 & -0.89643 & 1.00000 & & & \\
\hline Liu & 0.08666 & -0.44002 & -0.60583 & -0.03492 & 0.31161 & 1.00000 & & \\
\hline Turnover & -0.08900 & 0.44208 & 0.60491 & 0.03134 & -0.31002 & -0.99703 & 1.00000 & \\
\hline Bid Ask Spread & -0.60560 & 0.31113 & -0.55663 & -0.78753 & 0.85817 & 0.18548 & -0.18334 & 1.00000 \\
\hline \multicolumn{9}{|c|}{ Panel 30: Results for US (2006M05 - 2009M05) } \\
\hline & Price & Volatility & Volume & MV & Amihud & Liu & Turnover & Bid Ask Spread \\
\hline Price & 1.00000 & & & & & & & \\
\hline Volatility & -0.34240 & 1.00000 & & & & & & \\
\hline Volume & -0.20536 & 0.26021 & 1.00000 & & & & & \\
\hline MV & 0.50122 & -0.25983 & 0.54051 & 1.00000 & & & & \\
\hline Amihud & -0.48226 & 0.29713 & -0.60416 & -0.89164 & 1.00000 & & & \\
\hline Liu & 0.33174 & -0.59875 & -0.27931 & 0.41904 & -0.17345 & 1.00000 & & \\
\hline Turnover & -0.33322 & 0.58811 & 0.27959 & -0.42327 & 0.16672 & -0.98891 & 1.00000 & \\
\hline Bid Ask Spread & -0.43378 & 0.43074 & -0.07571 & -0.46534 & 0.48611 & -0.29185 & 0.29065 & 1.00000 \\
\hline \multicolumn{9}{|c|}{ Panel 31: Results for Australia (2001M07 - 2009M05) } \\
\hline & Price & Volatility & Volume & MV & Amihud & Liu & Turnover & Bid Ask Spread \\
\hline Price & 1.00000 & & & & & & & \\
\hline Volatility & -0.19582 & 1.00000 & & & & & & \\
\hline Volume & -0.25650 & -0.08932 & 1.00000 & & & & & \\
\hline MV & 0.54585 & -0.33647 & 0.47119 & 1.00000 & & & & \\
\hline Amihud & -0.51739 & 0.38664 & -0.58702 & -0.86608 & 1.00000 & & & \\
\hline $\mathrm{Liu}$ & 0.05840 & -0.19920 & -0.50430 & -0.01252 & 0.31961 & 1.00000 & & \\
\hline Turnover & -0.10794 & 0.24822 & 0.50634 & -0.04102 & -0.28305 & -0.93422 & 1.00000 & \\
\hline Bid Ask Spread & -0.53937 & 0.31211 & -0.46279 & -0.74782 & 0.84100 & 0.25814 & -0.24388 & 1.00000 \\
\hline \multicolumn{9}{|c|}{ Panel 32: Results for New Zealand (2001M01 - 2009M05) } \\
\hline & Price & Volatility & Volume & MV & Amihud & Liu & Turnover & Bid Ask Spread \\
\hline Price & 1.00000 & & & & & & & \\
\hline Volatility & -0.22929 & 1.00000 & & & & & & \\
\hline Volume & -0.26348 & 0.05697 & 1.00000 & & & & & \\
\hline MV & 0.59558 & -0.69877 & -0.06621 & 1.00000 & & & & \\
\hline Amihud & -0.41497 & 0.17573 & -0.72081 & -0.66995 & 1.00000 & & & \\
\hline Liu & -0.01361 & -0.02628 & -0.72300 & -0.15915 & 0.56982 & 1.00000 & & \\
\hline Turnover & -0.29495 & 0.04328 & 0.75679 & -0.11680 & -0.51582 & -0.87281 & 1.00000 & \\
\hline Bid Ask Spread & -0.44263 & 0.23130 & -0.53015 & -0.51291 & 0.77050 & 0.50075 & -0.45544 & 1.00000 \\
\hline
\end{tabular}




\begin{tabular}{|c|c|c|c|c|c|c|c|c|}
\hline \multicolumn{9}{|c|}{ Panel 33: Results for Argentina (2002M07 - 2009M05) } \\
\hline & Price & Volatility & Volume & MV & Amihud & Liu & Turnover & Bid Ask Spread \\
\hline Price & 1.00000 & & & & & & & \\
\hline Volatility & -0.17717 & 1.00000 & & & & & & \\
\hline Volume & -0.10584 & 0.24348 & 1.00000 & & & & & \\
\hline MV & 0.54324 & -0.04629 & 0.40833 & 1.00000 & & & & \\
\hline Amihud & -0.34751 & 0.02580 & -0.76596 & -0.61652 & 1.00000 & & & \\
\hline Liu & -0.03736 & -0.24252 & -0.84949 & -0.24209 & 0.73894 & 1.00000 & & \\
\hline Turnover & -0.22831 & 0.21971 & 0.52192 & -0.36262 & -0.32846 & -0.60451 & 1.00000 & \\
\hline Bid Ask Spread & -0.26552 & -0.02118 & -0.78919 & -0.55773 & 0.85706 & 0.79000 & -0.32573 & 1.00000 \\
\hline \multicolumn{9}{|c|}{ Panel 34: Results for Brazil (2001M01 - 2009M05) } \\
\hline & Price & Volatility & Volume & MV & Amihud & Liu & Turnover & Bid Ask Spread \\
\hline Price & 1.00000 & & & & & & & \\
\hline Volatility & 0.03536 & 1.00000 & & & & & & \\
\hline Volume & -0.10932 & 0.09681 & 1.00000 & & & & & \\
\hline MV & 0.50751 & -0.00707 & 0.24592 & 1.00000 & & & & \\
\hline Amihud & -0.28908 & 0.04641 & -0.64695 & -0.43922 & 1.00000 & & & \\
\hline Liu & 0.07828 & -0.06374 & -0.56304 & 0.34689 & 0.32780 & 1.00000 & & \\
\hline Turnover & 0.00854 & -0.00409 & 0.53354 & -0.30660 & -0.38786 & -0.92487 & 1.00000 & \\
\hline Bid Ask Spread & -0.09068 & -0.01757 & -0.63110 & -0.30317 & 0.66333 & 0.32239 & -0.34869 & 1.00000 \\
\hline \multicolumn{9}{|c|}{ Panel 35: Results for Chile (2002M07 - 2009M05) } \\
\hline & Price & Volatility & Volume & MV & Amihud & Liu & Turnover & Bid Ask Spread \\
\hline Price & 1.00000 & & & & & & & \\
\hline Volatility & -0.16139 & 1.00000 & & & & & & \\
\hline Volume & -0.44948 & 0.29832 & 1.00000 & & & & & \\
\hline MV & 0.43168 & -0.02531 & 0.34785 & 1.00000 & & & & \\
\hline Amihud & -0.29056 & 0.10235 & -0.47955 & -0.66623 & 1.00000 & & & \\
\hline Liu & -0.07636 & -0.26177 & -0.72655 & -0.53281 & 0.67433 & 1.00000 & & \\
\hline Turnover & -0.09736 & 0.26258 & 0.65103 & 0.03961 & -0.45675 & -0.63944 & 1.00000 & \\
\hline Bid Ask Spread & -0.25848 & 0.17570 & -0.22831 & -0.45576 & 0.46761 & 0.42520 & -0.17311 & 1.00000 \\
\hline \multicolumn{9}{|c|}{ Panel 36: Results for Colombia (1999M01 - 2001M12) } \\
\hline & Price & Volatility & Volume & MV & Amihud & Liu & Turnover & Bid Ask Spread \\
\hline Price & 1.00000 & & & & & & & \\
\hline Volatility & -0.19080 & 1.00000 & & & & & & \\
\hline Volume & -0.30229 & 0.22848 & 1.00000 & & & & & \\
\hline MV & 0.56978 & -0.09804 & -0.12792 & 1.00000 & & & & \\
\hline Amihud & -0.37011 & 0.38779 & -0.10538 & -0.22652 & 1.00000 & & & \\
\hline Liu & -0.10703 & -0.42826 & -0.44741 & -0.02035 & 0.15670 & 1.00000 & & \\
\hline Turnover & -0.09013 & 0.05276 & 0.54445 & -0.50548 & -0.18276 & -0.25872 & 1.00000 & \\
\hline Bid Ask Spread & -0.15162 & 0.06266 & -0.03826 & 0.04401 & 0.29112 & 0.31142 & -0.14013 & 1.00000 \\
\hline
\end{tabular}




\begin{tabular}{|c|c|c|c|c|c|c|c|c|}
\hline \multicolumn{9}{|c|}{ Panel 37: Results for Jamaica (2004M04 - 2009M05) } \\
\hline & Price & Volatility & Volume & MV & Amihud & Liu & Turnover & Bid Ask Spread \\
\hline Price & 1.00000 & & & & & & & \\
\hline Volatility & -0.23671 & 1.00000 & & & & & & \\
\hline Volume & -0.27262 & 0.02091 & 1.00000 & & & & & \\
\hline MV & 0.51844 & -0.30320 & 0.37086 & 1.00000 & & & & \\
\hline Amihud & -0.40502 & 0.42142 & -0.40677 & -0.64071 & 1.00000 & & & \\
\hline Liu & -0.11578 & 0.10754 & -0.73556 & -0.50563 & 0.59406 & 1.00000 & & \\
\hline Turnover & 0.00718 & 0.16395 & 0.40747 & -0.21928 & -0.12234 & -0.32791 & 1.00000 & \\
\hline Bid Ask Spread & -0.24838 & 0.45807 & -0.51057 & -0.69086 & 0.66349 & 0.68936 & 0.00811 & 1.00000 \\
\hline \multicolumn{9}{|c|}{ Panel 38: Results for Mexico (2002M07 - 2009M05) } \\
\hline & Price & Volatility & Volume & MV & Amihud & Liu & Turnover & Bid Ask Spread \\
\hline Price & 1.00000 & & & & & & & \\
\hline Volatility & -0.07130 & 1.00000 & & & & & & \\
\hline Volume & -0.09983 & 0.00419 & 1.00000 & & & & & \\
\hline MV & 0.51868 & -0.08707 & 0.49707 & 1.00000 & & & & \\
\hline Amihud & -0.26864 & 0.11683 & -0.83645 & -0.72227 & 1.00000 & & & \\
\hline Liu & 0.02133 & -0.07584 & -0.79588 & -0.18163 & 0.66883 & 1.00000 & & \\
\hline Turnover & -0.16780 & 0.05420 & 0.75060 & -0.02356 & -0.54801 & -0.87796 & 1.00000 & \\
\hline Bid Ask Spread & -0.16165 & 0.04158 & -0.80197 & -0.57284 & $\mathbf{0 . 8 3 4 8 3}$ & 0.69254 & -0.57687 & 1.00000 \\
\hline \multicolumn{9}{|c|}{ Panel 39: Results for Peru (2007M12 - 2009M05) } \\
\hline & Price & Volatility & Volume & MV & Amihud & Liu & Turnover & Bid Ask Spread \\
\hline Price & 1.00000 & & & & & & & \\
\hline Volatility & -0.12507 & 1.00000 & & & & & & \\
\hline Volume & -0.07168 & 0.08360 & 1.00000 & & & & & \\
\hline MV & 0.59230 & -0.22179 & 0.37966 & 1.00000 & & & & \\
\hline Amihud & -0.42187 & 0.22991 & -0.65199 & -0.59434 & 1.00000 & & & \\
\hline Liu & -0.18268 & -0.17283 & -0.80176 & -0.34094 & 0.64217 & 1.00000 & & \\
\hline Turnover & -0.30898 & 0.27943 & 0.32933 & -0.57383 & -0.06996 & -0.28658 & 1.00000 & \\
\hline Bid Ask Spread & -0.38556 & 0.07136 & -0.61332 & -0.57808 & 0.69124 & 0.70874 & 0.00309 & 1.00000 \\
\hline \multicolumn{9}{|c|}{ Panel 40: Results for Japan (2001M01 - 2009M05) } \\
\hline & Price & Volatility & Volume & MV & Amihud & Liu & Turnover & Bid Ask Spread \\
\hline Price & 1.00000 & & & & & & & \\
\hline Volatility & -0.0287 & 1.00000 & & & & & & \\
\hline Volume & -0.4580 & 0.1567 & 1.00000 & & & & & \\
\hline MV & 0.2889 & -0.0649 & 0.4215 & 1.00000 & & & & \\
\hline Amihud & -0.2338 & 0.0729 & -0.6655 & -0.7461 & 1.00000 & & & \\
\hline Liu & 0.0239 & -0.3826 & -0.5515 & 0.0100 & 0.4956 & 1.00000 & & \\
\hline Turnover & -0.0232 & 0.3855 & 0.5519 & -0.0133 & -0.4966 & -0.9950 & 1.00000 & \\
\hline Bid Ask Spread & -0.0836 & 0.1355 & -0.2417 & -0.2505 & 0.3693 & 0.1963 & -0.1942 & 1.00000 \\
\hline
\end{tabular}




\begin{tabular}{|c|c|c|c|c|c|c|c|c|}
\hline \multicolumn{9}{|c|}{ Panel 41: Results for Singapore (2004M02 - 2009M05) } \\
\hline & Price & Volatility & Volume & MV & Amihud & Liu & Turnover & Bid Ask Spread \\
\hline Price & 1.00000 & & & & & & & \\
\hline Volatility & -0.63559 & 1.00000 & & & & & & \\
\hline Volume & 0.05981 & 0.11149 & 1.00000 & & & & & \\
\hline MV & 0.81172 & -0.47661 & 0.35349 & 1.00000 & & & & \\
\hline Amihud & -0.69036 & 0.45964 & -0.64039 & -0.76123 & 1.00000 & & & \\
\hline Liu & -0.24674 & 0.05030 & -0.86293 & -0.44196 & 0.72335 & 1.00000 & & \\
\hline Turnover & 0.02890 & 0.10359 & 0.84831 & 0.08371 & -0.55103 & -0.75845 & 1.00000 & \\
\hline Bid Ask Spread & -0.75723 & 0.54976 & -0.49303 & -0.76088 & 0.87574 & 0.63906 & -0.41488 & 1.00000 \\
\hline \multicolumn{9}{|c|}{ Panel 42: Results for China Shenzen $(2002 \mathrm{M} 10-2009 \mathrm{M} 05)$} \\
\hline & Price & Volatility & Volume & MV & Amihud & Liu & Turnover & Bid Ask Spread \\
\hline Price & 1.00000 & & & & & & & \\
\hline Volatility & -0.02892 & 1.00000 & & & & & & \\
\hline Volume & -0.29780 & 0.30378 & 1.00000 & & & & & \\
\hline MV & 0.52147 & -0.08896 & 0.20277 & 1.00000 & & & & \\
\hline Amihud & -0.28824 & -0.01891 & -0.67379 & -0.57596 & 1.00000 & & & \\
\hline Liu & 0.18966 & -0.24347 & -0.38010 & 0.29075 & 0.15605 & 1.00000 & & \\
\hline Turnover & -0.29266 & 0.40093 & 0.58000 & -0.45346 & -0.28750 & -0.56907 & 1.00000 & \\
\hline Bid Ask Spread & -0.05755 & 0.01139 & -0.15230 & -0.06319 & 0.20482 & 0.10543 & -0.12922 & 1.00000 \\
\hline \multicolumn{9}{|c|}{ Panel 43: Results for China Shanghai (2007M09 - 2009M05) } \\
\hline & Price & Volatility & Volume & MV & Amihud & Liu & Turnover & Bid Ask Spread \\
\hline Price & 1.00000 & & & & & & & \\
\hline Volatility & 0.17925 & 1.00000 & & & & & & \\
\hline Volume & -0.43343 & -0.02015 & 1.00000 & & & & & \\
\hline MV & 0.16207 & -0.32678 & 0.45933 & 1.00000 & & & & \\
\hline Amihud & -0.15714 & 0.17407 & -0.70392 & -0.70851 & 1.00000 & & & \\
\hline Liu & 0.03964 & -0.31321 & -0.09381 & 0.37056 & -0.03186 & 1.00000 & & \\
\hline Turnover & -0.04716 & 0.50941 & 0.08323 & -0.67736 & 0.09633 & -0.55837 & 1.00000 & \\
\hline Bid Ask Spread & -0.10189 & 0.12925 & -0.04378 & -0.12474 & 0.12464 & -0.01702 & 0.06831 & 1.00000 \\
\hline \multicolumn{9}{|c|}{ Panel 44: Results for Hong Kong (2007M09- 2009M05) } \\
\hline & Price & Volatility & Volume & MV & Amihud & Liu & Turnover & Bid Ask Spread \\
\hline Price & 1.00000 & & & & & & & \\
\hline Volatility & -0.19258 & 1.00000 & & & & & & \\
\hline Volume & -0.29105 & 0.37546 & 1.00000 & & & & & \\
\hline MV & 0.57073 & -0.12651 & 0.32390 & 1.00000 & & & & \\
\hline Amihud & -0.42366 & -0.04556 & -0.66399 & -0.77089 & 1.00000 & & & \\
\hline Liu & 0.04054 & -0.45441 & -0.71078 & -0.06888 & 0.56529 & 1.00000 & & \\
\hline Turnover & -0.04816 & 0.49287 & 0.74510 & 0.06727 & -0.58385 & -0.94805 & 1.00000 & \\
\hline Bid Ask Spread & -0.32572 & -0.02704 & -0.58574 & -0.61570 & 0.82732 & 0.50201 & -0.51907 & 1.00000 \\
\hline
\end{tabular}




\begin{tabular}{|c|c|c|c|c|c|c|c|c|}
\hline \multicolumn{9}{|c|}{ Panel 45: Results for Indonesia (2007M09 - 2009M05) } \\
\hline & Price & Volatility & Volume & MV & Amihud & Liu & Turnover & Bid Ask Spread \\
\hline Price & 1.00000 & & & & & & & \\
\hline Volatility & -0.04119 & 1.00000 & & & & & & \\
\hline Volume & 0.17539 & 0.18569 & 1.00000 & & & & & \\
\hline MV & $\mathbf{0 . 7 7 8 3 1}$ & -0.07191 & 0.37774 & 1.00000 & & & & \\
\hline Amihud & -0.73718 & -0.00029 & -0.69268 & -0.77199 & 1.00000 & & & \\
\hline Liu & -0.22510 & -0.22712 & -0.74153 & -0.11752 & 0.57530 & 1.00000 & & \\
\hline Turnover & 0.19485 & 0.28770 & 0.77444 & 0.04732 & -0.55024 & -0.90781 & 1.00000 & \\
\hline Bid Ask Spread & 0.09736 & 0.06408 & -0.09705 & -0.00251 & 0.05206 & 0.07328 & -0.06038 & 1.00000 \\
\hline \multicolumn{9}{|c|}{ Panel 46: Results for Kazakhstan (2009M04 - 2009M05) } \\
\hline & Price & Volatility & Volume & MV & Amihud & Liu & Turnover & Bid Ask Spread \\
\hline Price & 1.00000 & & & & & & & \\
\hline Volatility & 0.14286 & 1.00000 & & & & & & \\
\hline Volume & -0.64286 & -0.42857 & 1.00000 & & & & & \\
\hline MV & 0.17857 & -0.32143 & 0.10714 & 1.00000 & & & & \\
\hline Amihud & -0.35714 & 0.39286 & -0.32143 & -0.35714 & 1.00000 & & & \\
\hline Liu & 0.25000 & 0.42857 & -0.82143 & -0.60714 & 0.57143 & 1.00000 & & \\
\hline Turnover & -0.10714 & -0.32143 & 0.21429 & -0.53571 & -0.32143 & 0.10714 & 1.00000 & \\
\hline Bid Ask Spread & -0.03571 & 0.67857 & -0.60714 & -0.71429 & 0.75000 & 0.89286 & 0.00000 & 1.00000 \\
\hline \multicolumn{9}{|c|}{ Panel 47: Results for Malaysia (2002M10 - 2009M05) } \\
\hline & Price & Volatility & Volume & MV & Amihud & Liu & Turnover & Bid Ask Spread \\
\hline Price & 1.00000 & & & & & & & \\
\hline Volatility & -0.48348 & 1.00000 & & & & & & \\
\hline Volume & 0.09403 & 0.17049 & 1.00000 & & & & & \\
\hline MV & 0.78766 & -0.33849 & 0.45249 & 1.00000 & & & & \\
\hline Amihud & -0.55655 & 0.23658 & -0.75397 & -0.76226 & 1.00000 & & & \\
\hline Liu & -0.01363 & -0.19566 & -0.79201 & -0.16159 & 0.58474 & 1.00000 & & \\
\hline Turnover & 0.01654 & 0.19989 & 0.81158 & 0.11893 & -0.58344 & -0.89246 & 1.00000 & \\
\hline Bid Ask Spread & -0.24071 & 0.14268 & -0.49453 & -0.39153 & 0.54847 & 0.41785 & -0.39761 & 1.00000 \\
\hline \multicolumn{9}{|c|}{ Panel 48: Results for Pakistan (2007M09 - 2009M05) } \\
\hline & Price & Volatility & Volume & MV & Amihud & Liu & Turnover & Bid Ask Spread \\
\hline Price & 1.00000 & & & & & & & \\
\hline Volatility & -0.20084 & 1.00000 & & & & & & \\
\hline Volume & -0.39709 & 0.29439 & 1.00000 & & & & & \\
\hline MV & 0.19746 & -0.11497 & 0.36158 & 1.00000 & & & & \\
\hline Amihud & 0.10890 & 0.07280 & -0.66984 & -0.39152 & 1.00000 & & & \\
\hline Liu & 0.25335 & -0.15330 & -0.77539 & -0.11685 & 0.77332 & 1.00000 & & \\
\hline Turnover & -0.06389 & 0.23604 & 0.80737 & 0.11239 & -0.65279 & -0.81397 & 1.00000 & \\
\hline Bid Ask Spread & 0.32718 & 0.03162 & -0.43064 & -0.20134 & 0.50607 & 0.47450 & -0.29312 & 1.00000 \\
\hline
\end{tabular}




\begin{tabular}{|c|c|c|c|c|c|c|c|c|}
\hline \multicolumn{9}{|c|}{ Panel 49: Results for Philippines (2007M11 - 2009M05) } \\
\hline & Price & Volatility & Volume & MV & Amihud & Liu & Turnover & Bid Ask Spread \\
\hline Price & 1.00000 & & & & & & & \\
\hline Volatility & -0.31581 & 1.00000 & & & & & & \\
\hline Volume & -0.21721 & 0.11800 & 1.00000 & & & & & \\
\hline MV & 0.62975 & -0.30310 & 0.17139 & 1.00000 & & & & \\
\hline Amihud & -0.42631 & 0.26522 & -0.64345 & -0.56498 & 1.00000 & & & \\
\hline Liu & -0.14166 & -0.02350 & -0.81295 & -0.24734 & 0.77734 & 1.00000 & & \\
\hline Turnover & -0.06545 & 0.15849 & 0.76359 & -0.16076 & -0.56256 & -0.75957 & 1.00000 & \\
\hline Bid Ask Spread & -0.29260 & 0.20735 & -0.60944 & -0.47123 & 0.73234 & 0.74560 & -0.45390 & 1.00000 \\
\hline \multicolumn{9}{|c|}{ Panel 50: Results for South Korea (2002M08 - 2009M05) } \\
\hline & Price & Volatility & Volume & MV & Amihud & Liu & Turnover & Bid Ask Spread \\
\hline Price & 1.00000 & & & & & & & \\
\hline Volatility & -0.14157 & 1.00000 & & & & & & \\
\hline Volume & -0.27999 & 0.30275 & 1.00000 & & & & & \\
\hline MV & 0.52431 & -0.01096 & 0.51670 & 1.00000 & & & & \\
\hline Amihud & -0.35182 & -0.05207 & -0.73780 & -0.87684 & 1.00000 & & & \\
\hline Liu & 0.28252 & -0.42098 & -0.60976 & 0.03068 & 0.35322 & 1.00000 & & \\
\hline Turnover & -0.29429 & 0.43231 & 0.61316 & -0.04474 & -0.34620 & -0.97331 & 1.00000 & \\
\hline Bid Ask Spread & -0.09414 & -0.09584 & -0.69554 & -0.58244 & 0.75206 & 0.42741 & -0.42095 & 1.00000 \\
\hline \multicolumn{9}{|c|}{ Panel 51: Results for Sri Lanka (2007M11 - 2009M05) } \\
\hline & Price & Volatility & Volume & MV & Amihud & Liu & Turnover & Bid Ask Spread \\
\hline Price & 1.00000 & & & & & & & \\
\hline Volatility & -0.18888 & 1.00000 & & & & & & \\
\hline Volume & -0.62340 & 0.01259 & 1.00000 & & & & & \\
\hline MV & -0.17689 & -0.16910 & 0.38163 & 1.00000 & & & & \\
\hline Amihud & 0.18361 & 0.15628 & -0.51544 & -0.26234 & 1.00000 & & & \\
\hline Liu & 0.47039 & 0.04940 & -0.67166 & -0.02338 & 0.48846 & 1.00000 & & \\
\hline Turnover & 0.14528 & 0.11185 & 0.27578 & -0.55585 & -0.22359 & -0.37649 & 1.00000 & \\
\hline Bid Ask Spread & 0.49583 & 0.16554 & -0.53315 & -0.33266 & 0.45732 & 0.61727 & 0.03681 & 1.00000 \\
\hline \multicolumn{9}{|c|}{ Panel 52: Results for Taiwan (2006M08 - 2009M05) } \\
\hline & Price & Volatility & Volume & MV & Amihud & Liu & Turnover & Bid Ask Spread \\
\hline Price & 1.00000 & & & & & & & \\
\hline Volatility & 0.00631 & 1.00000 & & & & & & \\
\hline Volume & -0.08620 & 0.19329 & 1.00000 & & & & & \\
\hline MV & 0.44776 & -0.08903 & 0.65905 & 1.00000 & & & & \\
\hline Amihud & -0.47025 & -0.01881 & -0.75000 & -0.88051 & 1.00000 & & & \\
\hline Liu & -0.04194 & -0.58479 & -0.36450 & 0.08037 & 0.29035 & 1.00000 & & \\
\hline Turnover & 0.04492 & 0.58469 & 0.36631 & -0.07756 & -0.29360 & -0.99778 & 1.00000 & \\
\hline Bid Ask Spread & -0.14207 & 0.00442 & -0.22285 & -0.25895 & 0.31156 & 0.12501 & -0.12598 & 1.00000 \\
\hline
\end{tabular}




\begin{tabular}{|c|c|c|c|c|c|c|c|c|}
\hline \multicolumn{9}{|c|}{ Panel 53: Results for Thailand (2001M08 - 2009M05) } \\
\hline & Price & Volatility & Volume & MV & Amihud & Liu & Turnover & Bid Ask Spread \\
\hline Price & 1.00000 & & & & & & & \\
\hline Volatility & -0.24803 & 1.00000 & & & & & & \\
\hline Volume & -0.45895 & 0.46574 & 1.00000 & & & & & \\
\hline MV & 0.64518 & -0.10548 & 0.09111 & 1.00000 & & & & \\
\hline Amihud & -0.35124 & -0.10645 & -0.56083 & -0.68264 & 1.00000 & & & \\
\hline Liu & 0.30057 & -0.45752 & -0.70873 & 0.20907 & 0.40246 & 1.00000 & & \\
\hline Turnover & -0.37159 & 0.49989 & 0.71230 & -0.29414 & -0.35060 & -0.93507 & 1.00000 & \\
\hline Bid Ask Spread & 0.20431 & -0.03014 & -0.25882 & 0.04104 & 0.12379 & 0.19248 & -0.19185 & 1.00000 \\
\hline \multicolumn{9}{|c|}{ Panel 54: Results for Vietnam (2009M04 - 2009M05) } \\
\hline & Price & Volatility & Volume & MV & Amihud & Liu & Turnover & Bid Ask Spread \\
\hline Price & 1.00000 & & & & & & & \\
\hline Volatility & -0.13934 & 1.00000 & & & & & & \\
\hline Volume & 0.04937 & 0.36585 & 1.00000 & & & & & \\
\hline MV & 0.65590 & 0.02892 & 0.55451 & 1.00000 & & & & \\
\hline Amihud & -0.38689 & -0.11797 & -0.84725 & -0.72191 & 1.00000 & & & \\
\hline Liu & 0.16834 & -0.43725 & -0.63988 & -0.00255 & 0.45497 & 1.00000 & & \\
\hline Turnover & -0.24686 & 0.42459 & 0.65787 & -0.11365 & -0.44961 & -0.86531 & 1.00000 & \\
\hline Bid Ask Spread & -0.07088 & 0.01356 & -0.37161 & -0.26126 & 0.43459 & 0.17745 & -0.20151 & 1.00000 \\
\hline \multicolumn{9}{|c|}{ Panel 55: Results for Abu Dhabi (2007M12 - 2009M05) } \\
\hline & Price & Volatility & Volume & MV & Amihud & Liu & Turnover & Bid Ask Spread \\
\hline Price & 1.00000 & & & & & & & \\
\hline Volatility & -0.11273 & 1.00000 & & & & & & \\
\hline Volume & -0.28176 & -0.15812 & 1.00000 & & & & & \\
\hline MV & 0.28189 & -0.27171 & 0.54103 & 1.00000 & & & & \\
\hline Amihud & -0.03391 & 0.41324 & -0.75321 & -0.61405 & 1.00000 & & & \\
\hline Liu & 0.24662 & 0.07681 & -0.86587 & -0.39914 & 0.69579 & 1.00000 & & \\
\hline Turnover & -0.19718 & -0.06693 & 0.77703 & 0.10774 & -0.56539 & -0.66532 & 1.00000 & \\
\hline Bid Ask Spread & 0.17324 & 0.25041 & -0.67352 & -0.42369 & 0.62975 & 0.69416 & -0.45898 & 1.00000 \\
\hline \multicolumn{9}{|c|}{ Panel 56: Results for Dubai (2008M01 - 2009M05) } \\
\hline & Price & Volatility & Volume & MV & Amihud & Liu & Turnover & Bid Ask Spread \\
\hline Price & 1.00000 & & & & & & & \\
\hline Volatility & -0.14199 & 1.00000 & & & & & & \\
\hline Volume & -0.43445 & 0.11721 & 1.00000 & & & & & \\
\hline MV & 0.36470 & -0.23944 & 0.38557 & 1.00000 & & & & \\
\hline Amihud & 0.23719 & 0.03656 & -0.92378 & -0.51789 & 1.00000 & & & \\
\hline Liu & 0.49134 & -0.21302 & -0.87060 & -0.08573 & 0.77011 & 1.00000 & & \\
\hline Turnover & -0.49578 & 0.20650 & 0.83203 & -0.04038 & -0.74218 & -0.94612 & 1.00000 & \\
\hline Bid Ask Spread & 0.28391 & 0.04586 & -0.84968 & -0.42000 & 0.86542 & 0.75974 & -0.71059 & 1.00000 \\
\hline
\end{tabular}




\begin{tabular}{|c|c|c|c|c|c|c|c|c|}
\hline \multicolumn{9}{|c|}{ Panel 57: Results for Israel (2002M12 - 2009M05) } \\
\hline & Price & Volatility & Volume & MV & Amihud & Liu & Turnover & Bid Ask Spread \\
\hline Price & 1.00000 & & & & & & & \\
\hline Volatility & -0.03102 & 1.00000 & & & & & & \\
\hline Volume & -0.48419 & -0.08125 & 1.00000 & & & & & \\
\hline MV & 0.11074 & -0.23626 & 0.62810 & 1.00000 & & & & \\
\hline Amihud & -0.15166 & 0.23419 & -0.68122 & -0.86212 & 1.00000 & & & \\
\hline Liu & -0.05236 & -0.07860 & -0.62011 & -0.42767 & 0.68141 & 1.00000 & & \\
\hline Turnover & 0.04734 & 0.10970 & 0.57987 & 0.34203 & -0.63950 & -0.94437 & 1.00000 & \\
\hline Bid Ask Spread & -0.09959 & 0.17434 & $-\mathbf{0 . 7 0 7 3 0}$ & -0.82477 & 0.90563 & 0.68349 & -0.61994 & 1.00000 \\
\hline \multicolumn{9}{|c|}{ Panel 58: Results for Jordan (2009M02 - 2009M05) } \\
\hline & Price & Volatility & Volume & MV & Amihud & Liu & Turnover & Bid Ask Spread \\
\hline Price & 1.00000 & & & & & & & \\
\hline Volatility & -0.19809 & 1.00000 & & & & & & \\
\hline Volume & -0.19344 & 0.41583 & 1.00000 & & & & & \\
\hline MV & 0.53419 & -0.15713 & 0.11860 & 1.00000 & & & & \\
\hline Amihud & -0.11294 & -0.15410 & -0.82693 & -0.37645 & 1.00000 & & & \\
\hline Liu & 0.23635 & -0.35093 & -0.81423 & 0.07864 & 0.72143 & 1.00000 & & \\
\hline Turnover & -0.27273 & 0.48406 & 0.86270 & -0.29303 & -0.59683 & -0.76906 & 1.00000 & \\
\hline Bid Ask Spread & -0.31392 & 0.43244 & -0.10821 & -0.30425 & 0.38638 & 0.20287 & 0.05648 & 1.00000 \\
\hline \multicolumn{9}{|c|}{ Panel 59: Results for Kuwait (2009M02 - 2009M05) } \\
\hline & Price & Volatility & Volume & MV & Amihud & Liu & Turnover & Bid Ask Spread \\
\hline Price & 1.00000 & & & & & & & \\
\hline Volatility & -0.73861 & 1.00000 & & & & & & \\
\hline Volume & -0.35479 & 0.47940 & 1.00000 & & & & & \\
\hline MV & 0.59424 & -0.39228 & 0.18572 & 1.00000 & & & & \\
\hline Amihud & -0.23897 & 0.04921 & -0.67492 & -0.56611 & 1.00000 & & & \\
\hline Liu & 0.21152 & -0.43085 & -0.86837 & -0.21527 & 0.68414 & 1.00000 & & \\
\hline Turnover & -0.41205 & 0.48031 & 0.85323 & -0.19542 & -0.47875 & -0.70770 & 1.00000 & \\
\hline Bid Ask Spread & -0.03773 & -0.06161 & -0.63034 & -0.40568 & 0.64190 & 0.65612 & -0.42731 & 1.00000 \\
\hline \multicolumn{9}{|c|}{ Panel 60: Results for Oman (2009M02 - 2009M05) } \\
\hline & Price & Volatility & Volume & MV & Amihud & Liu & Turnover & Bid Ask Spread \\
\hline Price & 1.00000 & & & & & & & \\
\hline Volatility & -0.63291 & 1.00000 & & & & & & \\
\hline Volume & -0.40586 & 0.38777 & 1.00000 & & & & & \\
\hline MV & 0.71185 & -0.55245 & -0.17499 & 1.00000 & & & & \\
\hline Amihud & -0.26609 & 0.19145 & -0.46670 & -0.34154 & 1.00000 & & & \\
\hline Liu & 0.47067 & -0.38932 & -0.70464 & 0.55632 & 0.23356 & 1.00000 & & \\
\hline Turnover & -0.48467 & 0.55013 & 0.62886 & -0.74500 & -0.06913 & -0.79191 & 1.00000 & \\
\hline Bid Ask Spread & -0.09362 & 0.05304 & -0.48626 & -0.13348 & 0.50748 & 0.41039 & -0.21251 & 1.00000 \\
\hline
\end{tabular}




\begin{tabular}{|c|c|c|c|c|c|c|c|c|}
\hline \multicolumn{9}{|c|}{ Panel 61: Results for Qatar (2008M10 - 2009M05) } \\
\hline & Price & Volatility & Volume & MV & Amihud & Liu & Turnover & Bid Ask Spread \\
\hline Price & 1.00000 & & & & & & & \\
\hline Volatility & 0.02190 & 1.00000 & & & & & & \\
\hline Volume & -0.41026 & 0.02926 & 1.00000 & & & & & \\
\hline MV & 0.54789 & 0.06339 & 0.19585 & 1.00000 & & & & \\
\hline Amihud & 0.05052 & 0.08434 & -0.87567 & -0.43622 & 1.00000 & & & \\
\hline Liu & 0.51319 & 0.06232 & -0.73439 & 0.21786 & 0.58016 & 1.00000 & & \\
\hline Turnover & -0.60394 & 0.00146 & 0.73479 & -0.39746 & -0.52914 & -0.84393 & 1.00000 & \\
\hline Bid Ask Spread & 0.17086 & -0.00317 & -0.83593 & -0.26999 & 0.85052 & 0.66129 & -0.58756 & 1.00000 \\
\hline \multicolumn{9}{|c|}{ Panel 62: Results for Saudi Arabia (2009M02 - 2009M05) } \\
\hline & Price & Volatility & Volume & MV & Amihud & Liu & Turnover & Bid Ask Spread \\
\hline Price & 1.00000 & & & & & & & \\
\hline Volatility & -0.00977 & 1.00000 & & & & & & \\
\hline Volume & -0.67120 & 0.27204 & 1.00000 & & & & & \\
\hline MV & 0.11396 & -0.46471 & -0.16577 & 1.00000 & & & & \\
\hline Amihud & 0.25388 & 0.00018 & -0.72201 & -0.06439 & 1.00000 & & & \\
\hline Liu & 0.24038 & -0.55167 & -0.56546 & 0.85639 & 0.32966 & 1.00000 & & \\
\hline Turnover & -0.23838 & 0.55179 & 0.56389 & -0.85828 & -0.32848 & -0.99912 & 1.00000 & \\
\hline Bid Ask Spread & 0.08517 & -0.15116 & -0.32446 & 0.14375 & 0.36359 & 0.29317 & -0.29553 & 1.00000 \\
\hline \multicolumn{9}{|c|}{ Panel 63: Results for Turkey (2009M02-2009M05) } \\
\hline & Price & Volatility & Volume & MV & Amihud & Liu & Turnover & Bid Ask Spread \\
\hline Price & 1.00000 & & & & & & & \\
\hline Volatility & -0.15118 & 1.00000 & & & & & & \\
\hline Volume & -0.43372 & 0.24798 & 1.00000 & & & & & \\
\hline MV & 0.62093 & -0.08257 & 0.19601 & 1.00000 & & & & \\
\hline Amihud & -0.17916 & -0.02037 & -0.72549 & -0.65140 & 1.00000 & & & \\
\hline Liu & 0.63038 & -0.30418 & -0.58504 & 0.51984 & 0.17845 & 1.00000 & & \\
\hline Turnover & -0.63816 & 0.31235 & 0.60807 & -0.52007 & -0.19499 & -0.97446 & 1.00000 & \\
\hline Bid Ask Spread & 0.05920 & 0.04453 & -0.10366 & -0.03153 & 0.12756 & 0.09462 & -0.09498 & 1.00000 \\
\hline
\end{tabular}




\section{Appendix 3. Vuong likelihood ratio test}

Following Lesmond (2005) a likelihood ratio test is used that was originally proposed by Vuong (1989). This is specific for non-nested model selection in testing whether a reference model and comparison model do equally well at explaining the underlying data. The reference model is either the Amihud measure (Amihud 2002) or alternatively the Turnover measure, which is a measure of trading frequency, and the comparison models are the remaining liquidity measures, i.e. Liu derived from Liu (2006) and the liquidity determinants of Stoll (2000). Each of the comparison models are tested independently against the reference Amihud or Turnover measure with each individual regression stated as:

Reference Model: $\quad \mathbf{S}+\mathrm{C}_{\mathrm{j}}=\theta_{0}+\theta_{1}\left(\right.$ reference liquidity measure $\left.{ }_{\mathrm{j}}\right)+\boldsymbol{\varepsilon}_{\mathrm{j}}$,

Comparison Model 1: $S+C_{j}=\gamma_{0}+\gamma_{1}$ other liquidity measure $(s)_{j}+\varepsilon_{j}$, and

Comparison Model 2: $\mathbf{S}+\mathrm{C}_{\mathrm{j}}=\gamma_{0}+\gamma_{1}$ price $_{\mathrm{j}}+\gamma_{2}$ volume $_{\mathrm{j}}+\gamma_{3} \sigma_{\mathrm{j}}^{2}+\gamma_{4}$ size $_{\mathrm{j}}+\varepsilon_{\mathrm{j}}$,

where $\mathrm{S}+\mathrm{C}_{\mathrm{j}}$ refers to the average, proportional bid ask spread plus commission cost for each firm-month $\mathrm{j}$ within each country. The comparison liquidity measures are those of Liu (2006). Price is the average annual daily stock price in local currency, and volume is the average annual daily trading volume. $\sigma_{\mathrm{j}}^{2}$ is the daily average volatility. Size is the monthly market capitalization and is defined as the share price times the number of shares outstanding. Share price is measured at the beginning of each month, while number of shares outstanding is measured at the beginning of the year.

The basis of the test is a likelihood ratio of the log likelihood function for the reference model to the log likelihood function for the comparison model. Using $\mathrm{R}$ to represent the reference model and $\mathrm{C}$ to represent the comparison model:

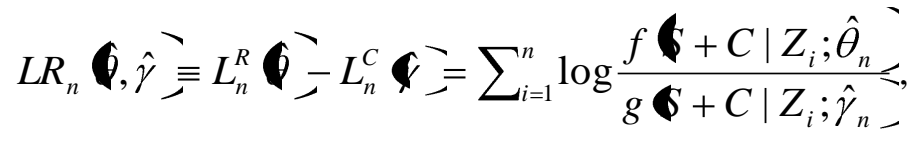

Where $\mathrm{LR}_{\mathrm{n}}$ is the likelihood ratio function for $\mathrm{n}$ firm-month observations in each country. $\mathrm{Z}_{\mathrm{i}}$ is a vector of $\mathrm{m}$ independent standard normal variables, $\hat{\theta}_{n}$ is the maximum likelihood parameter estimates for the reference model, and $\hat{\gamma}_{n}$ is the maximum likelihood parameter estimates for the comparison model. The variance of the likelihood function is given by Vuong as

$$
\hat{\omega}_{n}^{2}=\frac{1}{n} \sum_{j}^{n}\left(\frac{1}{2}\left[\log \hat{\mathbf{\sigma}}_{C}^{2}-\log \hat{\mathbf{\sigma}}_{R}^{2}\right]+\frac{1}{2}\left[\frac{\varepsilon_{C j}^{2}}{\hat{\sigma}_{C}^{2}}-\frac{\varepsilon_{R j}^{2}}{\hat{\sigma}_{R}^{2}}\right]\right)-\left[\frac{1}{2} L R_{n}\right]^{2}
$$


where $\varepsilon$ is the residual using the fitted parameters for either the Amihud regression case of the comparison model case. Vuong shows that the likelihood ratio statistic converges to a normal distribution:

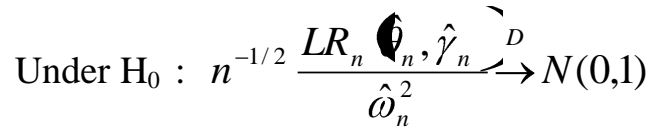

The resultant test statistic is stated as

$$
Z=\frac{1}{\sqrt{n}} \frac{L R_{n} \hat{\mathbf{Q}}_{n}, \hat{\gamma}_{n}}{\hat{\omega}_{n}}
$$

A one-sided Z-statistic tests whether either of the reference models, in this case being either the Amihud or Turnover measure, is more highly associated with the underlying $\mathrm{S}+\mathrm{C}$ cost than the comparison model(s). The test is directional, given by a positive or negative $\mathrm{Z}$ statistic, indicating which model is more highly associated with the underlying $\mathrm{S}+\mathrm{C}$ cost. A positive and significant Z-statistic indicates that the reference measure is more highly associated with the underlying $\mathrm{S}+\mathrm{C}$ cost than the comparison measure(s). A negative and significant Z-statistic indicates the comparison measure is more highly associated with the underlying $\mathrm{S}+\mathrm{C}$ cost.

Alternatively, the Z-statistic can be obtained from a linear regression if the log ratio is defined at every month $j$ as

$$
m_{j}=\frac{1}{2} \log \left[\frac{\hat{\sigma}_{C}^{2}}{\hat{\sigma}_{R}^{2}}\right]+\frac{1}{2}\left[\frac{\varepsilon_{C j}^{2}}{\hat{\sigma}_{C}^{2}}-\frac{\varepsilon_{R j}^{2}}{\hat{\sigma}_{R}^{2}}\right]
$$

Vuong states that a useful abstraction of the test statistic in above equation " $\frac{1}{\sqrt{n}} \frac{L R_{n} \boldsymbol{\phi}_{n}, \hat{\gamma}_{n}}{\hat{\omega}_{n}}$ is numerically equal to $[(n-1) / n]^{1 / 2}$ times either the usual t-statistic on the constant term in a linear regression of $m_{j}$ on only the constant term, or the usual t-statistic on the coefficient of $\mathrm{m}_{\mathrm{j}}$ in a linear regression of one on $\mathrm{m}_{\mathrm{j}}$." Stated another way, the Z-statistic can be obtained by regressing $m_{j}$ on unity and multiplying the $t$-statistic from this regression by $[(n-1) / n]^{1 / 2}$. This procedure involving the running of two subsequent sets of regressions is employed in this paper. 